

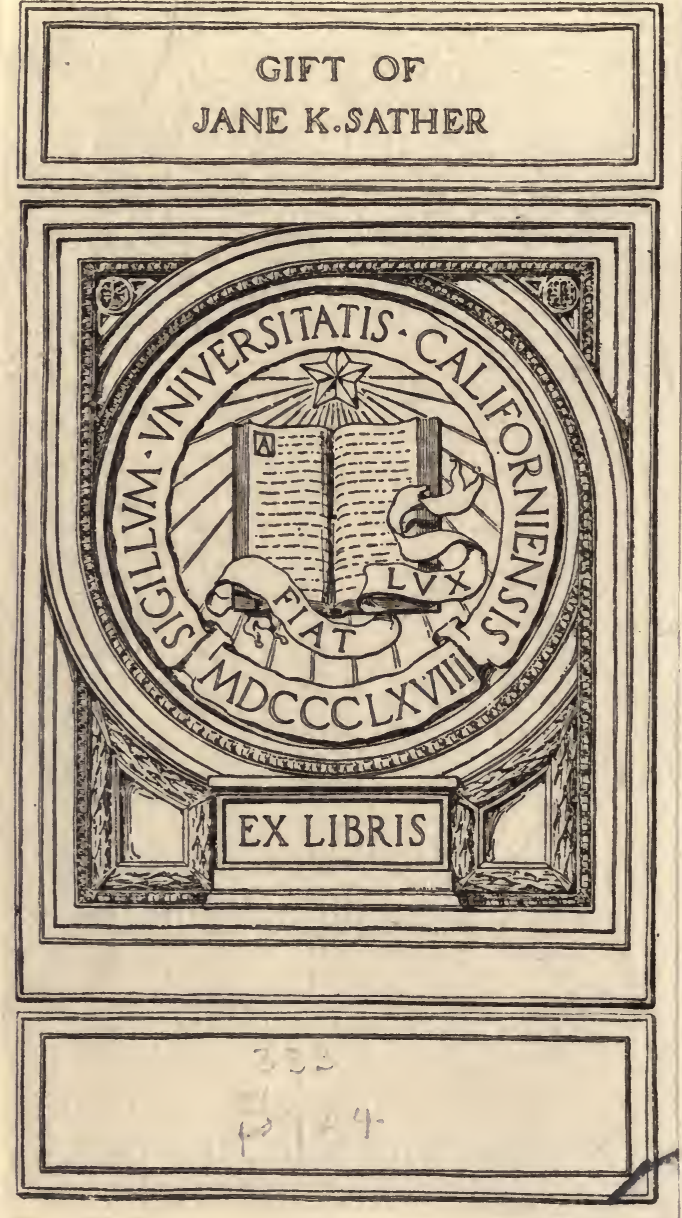


\section{LECTURES ON THE}

METHOD OF SCIENCE

EDITED BY

T. B. ST T R O N G
DEAN OF CHRIST CHURCH

OXFORD

AT THE CLARENDON PRESS

1906 
HENRY FROWDE, M.A. FUBLISHER TO THE UNIVERSITY OF OXFORD I.ONDON, EDINBURGH NEW YORK AND TORONTO

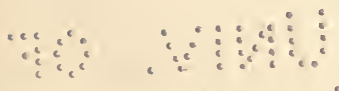

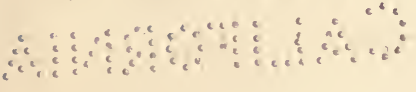




\section{PREFACE}

THE Lectures published in the present volume form part of a course on Scientific Method, delivered at the request of the Delegates for the Extension of University Teaching during the Summer Meeting, at Oxford, in August, 1905.

At previous meetings it had been the practice to offer, in the section of Natural Science, a course of lectures in some special branch of scientific study. When the arrangements for the meeting of 1905 were under discussion, a suggestion was made to the Delegates by $\mathrm{Mr}$. A. W. Brown-formerly Exhibitioner of Christ Church, and now Principal of the University Extension College at Stafford-to the effect that a different plan should be pursued this year. Instead of a course upon some particular branch of scientific investigation, it was suggested that a course should be given illustrating the forms taken by scientific method in various departments of research. The suggestion was approved by the Delegates, and a scheme of Lectures was drawn up. The course as delivered in Oxford was received with great satisfaction, and it appeared to the Delegates that it might be of value to publish some of the Lectures in the hope that their high interest, both in themselves and in their mutual connexion, would justify their presentation to a wider public.

It is unnecessary to point out that the scheme is a difficult one in itself, and was rendered still more so by 
the fact that the Lecturers, some of whom were not resident in Oxford, had no opportunity of formal conference beforehand as to the exact points to be dealt with by each. They had the scheme before them, and general suggestions as to the purpose of the course, and nothing more. A few words may, therefore, be permitted as to the way in which the lectures now published illustrate the scheme. The first two Lectures, which were introductory to the whole course, describe in general terms the aim and character of scientific method from two slightly different points of view. Professor Case shows the various forms in which the general material of scientific study is exhibited: the various logical processes by which the facts under investigation are co-ordinated and explained. Professor Gotch, on the other hand, draws from an account of an historic incident in the warfare between science and ignorance, the true temper and aims of the scientific man : his relentless criticism of authority, his appeal to reason alone, his refusal to regard any fact as unimportant or meaningless: together with his bold but cautious use of the imagination for the extension and consolidation of knowledge.

From this point we pass to the special sciences: and here two methods were possible. The Lecturers might have produced what would have been, in strictness, logical dissertations-discussions on the method of science with illustrations from their special department. The alternative plan was to describe some particular investigation belonging to each special science, and to show the method of the science emerging, as it were, from the description of 
the facts concerned, the way in which they are approached, and the degree and character of the certainty attained. The second of these two plans was the one chosen. Professor Sherrington illustrates by an account of the problem of the warmth of the body his description of Physiology 'as the study of the working of living things'. Professor Weldon shows the difficulties with which the student has to contend who aims at giving an accurate and trustworthy interpretation of the facts involved in Inheritance. Mr. McDougall explains, by means of an account of the methods of determining the mode and duration of the perception of light, some of the ways in which it is attempted to bring scientific accuracy into the region of psychical phenomena under the head of non-experimental sciences. Dr. Fison shows how the methods of physics may be used to explain phenomena observed in stars far beyond the reach of the finest telescope. In the other three Lectures we deal with the phenomena presented by man in his history in the world. Sir Richard Temple by his sketch of the development of currency shows how principles, which we all still apply and understand, have developed in logical order, and have operated in the whole history of man. Professor Flinders Petrie shows how man registers the history of his own social development, unconsciously in the things he makes and uses and leaves behind him when he dies. And in the last Lecture an attempt is made to show upon what we rest our confidence in history, when 'material history' fails us.

It is obvious that in a course like this the whole ground 
of scientific inquiry is not and could not be covered. The Lectures merely exhibit types of the way in which different classes of facts are approached, the special difficulties attaching to them surmounted, and something like trustworthy accuracy attained in regard to them. In this limited but important way, it is hoped that the book may have its use.

The Delegates desire to express their sincere thanks to Mr. Brown for his suggestion, and to Mr. J. L. Myres, M.A., Student of Christ Church, for his energetic assistance in working out the scheme. 


\section{CONTENTS}

\section{LECTURE I}

Scientific Method as a Mental Operation .

(Thomas CASE, M.A., President of Corpus Christi College, and Waynflete Professor of Moral and Metaphysical Philosophy at Oxford)

LECTURE II

On some Aspects of the Scientific Method.

(Francis Gotch, D.Sc., F.R.S., Waynflete Professor of Physiology at Oxford)

\section{LECTURE III}

Physiology; its Scope AND Method

(C. S. Sherrington, D.Sc., LL.D., F.R.S., \&c., Pro-

fessor of Physiology, University of Liverpool)

LECTURE IV

Inheritance in ANimals aNd Plants

(W. F. R. Weldon, D.Sc., F.R.S., Linacre Professor of Comparative Anatomy at Oxford)

\section{LECTURE V}

Psycho-Physical Method

(W. McDougall, M.A., Wilde Reader in Mental Philosophy at Oxford)

LECTURE VI

The Evolution of Double Stars (A. H. Fison, D.Sc.) 


\section{LECTURE VII}

Anthropology: The Evolution of CurRency and

COINAGE

(Sir Richard C. Temple, Bart., G.C.S.I., D.C.L.)

\section{LECTURE VIII}

Archaeological Evidence .

(W. M. Flinders Petrie, D.C.L., F.R.S., Professor of Egyptology, University College, London)

LECTURE IX

Scientific Method as applied to History

(The Very Rev. T. B. Strong, D.D., Dean of Christ

Church) 


\section{SCIENTIFIC METHOD AS A MENTAL OPERATION ${ }^{1}$}

(Thomas CASE)

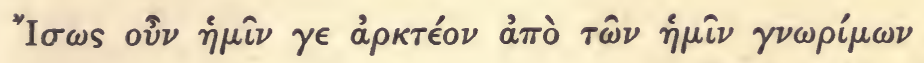

(ARISTOTLE)

Scientific Method is simply the way in which inferences are arranged in any science., We must therefore begin by saying something about inferences. The first definite classification of inference was due to Aristotle, who discerned that sometimes we infer from particular to particular, sometimes from particular to universal, sometimes from universal to particular ${ }^{2}$. These three kinds of inference are what we now call analogical, inductive and deductive. When I conclude that, as this and that body press, so does another body, I infer from particular to particular by analogy, or what Aristotle called 'example': when I conclude that, as this and that body press, so do all bodies, I infer from particular to universal by induction : when I infer that, as all bodies press, so does this body, I infer from universal to par-

1 The gist of this Lecture was delivered from notes. When something had to be written out for the Press, the writer found himself unable to compress it into the compass of an hour's lecture. Even in its extended form, it does not say enough about imagination and opinion in relation to science.
2 Aristotle, Prior Analytics, ii, 24.

S. L. 
ticular by what was called by Aristotle syllogism, but more often nowadays deduction.

These three kinds of inference agree in consisting of judgements or beliefs; in proceeding from judgements as premisses to another judgement as conclusion; and in performing this process by a perception of similarity between what is in the premisses and what is in the conclusion. Analogy concludes from one particular being like another, induction from a class being like its instances, and deduction from any member being like all the members of a class. 'Inference in general then is proceeding from judgement to judgement by means of similarity, or what is often called 'parity of reasoning'.

At the same time, there are minor differences between the kinds of inference. Analogy requires nothing but similar particulars. It is the kind of inference which may be performed by the higher animals, and by young children, without having risen to the universal, without any general apprehension of a whole class, and without any general terms or rational language. Mill did an important service in insisting on this inference from particular to particular, by which a dog or an infant will argue from one meal to another, without understanding the whole class of meals, or expressing that class by any general term. On the other hand, we must distinguish. -more sharply than he did" between this particular inference of analogy and the general inferences of induction and deduction, which involve the apprehension of whole classes, and are bound up with the use of general language or rational discourse. Induction and deduction imply generalization. They are reasoning. They are the privileges of man, who alone in the animal world uses the word 'all', who alone generalizes, or has reason proper. Beasts infer : man reasons., 
Moreover, within rational inferences there are further differences between induction and deduction, but especially that induction leaves us in a mere state of indefinite generality, while deduction brings us down to definite particulars., Take, for example, the prediction of eclipses. Induction will tell us that, whenever an opaque body intervenes between an illuminating and an illuminated body, there will be an eclipse. But to apply this mere generality, deduction is required to combine it as a major premiss with a particular as a minor premiss that, on a particular day, hour, and minute, a body, say the earth, will intervene between an illuminating and an illuminated body, say sun and moon, and so to conclude that there will be an eclipse of the latter at this particular time. In short, it is the function of man as a rational animal to emerge from particular to general inference, or from analogy to reasoning, and therein to advance from induction to deduction. As, too, action always takes place in particulars, induction must be followed by deduction in order to carry our knowledge of laws into practice; or, as Comte said, 'induire pour déduire afin de construire.'s

All three kinds of inference can give us knowledge only by starting from experience, however indirect this may afterwards become. All three, however little they go beyond their starting-point, nevertheless carry the mind beyond experience. Even analogy goes beyond experience, as when a dog infers that he is going to get his dinner before he gets it. General inference goes farther: it rises to universals beyond experience; for nobody can experience all the members of a class, or all the cases of a law in nature. What goes furthest of all is deduction, because it carries the mind from universals to new particulars beyond experience; in the 
future, as when we deduce a particular eclipse; in the past, as when we deduce a particular animal from fossil remains : in the present, as when we deduce that this glass of water contains an innumerable number of molecules of oxygen and hydrogen. 'It is not by induction but by deduction that we know definite particulars beyond experience in the future, in the past, and even in the present.s

- All inference then begins from, and proceeds beyond, experience. Yet it is common among philosophers to reduce knowledge to mere experience, and define science as systematic experience. System, yes : the very difference between ordinary and scientific knowledge is that while the former is sporadic, the latter is systematic. As Aristotle said, every science selects a subject, such as number in arithmetic, magnitudes in geometry, investigates as many propositions of its subject as possible, and aims at last in bringing the multitude of propositions under a few principles, in order to convert its whole knowledge of the subject into a system. 'Science then is systematization. But it is not mere systematic experience, but also systematic inference from, and beyond, experience. . The science of the moon, for example, must start from its visible face. But, as the moon rotates only once in a revolution round the earth, it shows us only one face, of which alone we have experience. How then do we know that it has another side always turned from us, and in fact that it is a sphere? Not by experience, but by inference from experience of other spheres. ${ }^{2}$ Science then is systematic ${ }_{k}$ knowledge by experience and inference.:

Now ${ }^{c}$ scientific method is merely the way, or ways, of using different orders of inference in investigating any subject of science with a view to its system., Sometimes, 
indeed, we have to be content with mere analogy. Sometimes we can use induction, and sometimes deduction. There is no one rule. For, if man shows to advantage in the possession of reason, he is equally at a disadvantage in his scientific investigation of Nature. Man was, as it were, born too late. He came into the world long after the beginning; in a very small world, in a planet of the sun which is not a star of the first magnitude; when this planet had gone through much evolution before it became adapted to him; and within the limits of a puny organism, with imperfect senses, which are however his only direct avenues to the external universe, and therefore the necessary conditions of his reason. In these circumstances he has to fasten on Nature as best he can. He knows more about present $x$. facts than about past causes; and when he knows causes, it is sometimes the efficient cause, as in the mechanics of the forces by which bodies act on one another; sometimes the material cause, as when the chemist knows the elements out. of which a body is composed without having discovered the force of chemical affinity so-called by which they compose it; sometimes again the final cause, as in morals, where we know for what end we act without fully understanding the voluntary, nervous, and muscular forces by which we perform our actions.

Aristotle perceived this defect of human science when he enunciated the fundamental principle of methodWe must proceed from the better known to us. As what is best known to us is various, the starting-point is + various, the processes of inference are various, and therefore scientific methods are various. Aristotle long ago said that methods vary with the subject-matter. Mill, the best logician of our times, may be said to have written the commentary on this text. He distinguishes 
the mathematical, the empirical, the explanatory, and the verificatory method, and shows to what subjects each of these four methods must be applied. We shall roughly follow him, but endeavour also to show that his account of deductive methods is imperfect.

The first method is the mathematical, which consists $\checkmark$ in the main of principles and deductions about numbers in arithmetic and algebra, and about magnitudes in geometry. Mathematical principles are so obvious as to seem a priori. It is so quickly perceived that one and one are two, and a triangle is a three-sided rectilineal figure, that philosophers have been led to suppose that the mind knows these principles by adding something to experience. Connected with this a priori hypothesis is another-a constructive hypothesis that the mind beginning arithmetic with abstract units constructs numbers, and beginning geometry with points constructs lines, surfaces and solids of three dimensions, and might according to some have constructed magnitudes with less or more dimensions. These views have the history of mathematics against them. Man began arithmetic with experience of the number of his fingers and toes, and geometry with experience of the magnitude of his hands, feet, and arms. He went on to use these concrete bodies as standards to measure other bodies. Geometry means the measurement of lands; and the most ancient Egyptian book of mathematics, the papyrus of Ahmes, about I 700 B.C. ${ }^{1}$, measures barns, pyramids, and obelisks, and treats solid bodies before proceeding to abstract surfaces. Mathematics, in short, began with concrete bodies, such as could only be reached by means of experience, and only gradually receded

${ }^{1}$ See Gow's History of Greek Mathematics, pp. I 6 seq., 126 seq. 
from the concrete to the abstract, to the units of abstract arithmetic, and the points of abstract geometry. The Greeks achieved this analysis from concrete to abstract, and thus converted mathematics from analysis to synthesis, which begins with the abstract unit as origin of number, and with the abstract point as simpler than the line. But the order of discovery was from the concrete and analytical, although afterwards the order of development was from the abstract and synthetic. Nor did the certainty of mathematical principles require an a priori origin. They are equations, or rather identical judgements ; and, after experience of their objects, the mind has the power of perceiving their identity. Thus once we have experienced two we perceive that it is the same as one and one; having experienced a triangle we perceive that there is no difference between it and a three-sided rectilineal figure; after experience of wholes we perceive that a whole must be greater than a part of it, otherwise it would not be a whole. Nothing is absolutely necessary except that a thing is itself. "The method then of mathematics consists of principles, discovered from experience and apprehended as certain by the power of perceiving identity, and of demonstrations or deductions of consequences from these identical and therefore necessary, principles. ,

Though we have the mathematical power of apprehending identical certainty, and demonstrating consequent certainties, this power is limited. It requires simple objects, such as numbers and figures, which after experience admit of great abstraction, and of such simplification that we are able to grasp the whole abstract object and so apprehend with certainty what it identically is. We may indeed extend this power of abstract simplification and identification from mathematics to physics, 
which is based on the identical principle-bodies as interresistant, or mutually impenetrable, must force one another apart. But as science proceeds, simplicity becomes a snare. Nature is complex. The complexity of nature, which is the limit of mathematical method, requires us to go back to the experience of concrete things. The merit of ancient science was its mathematical form : its weakness was its mathematical exaggeration, feigning simplicity and demonstration, when the former was absent and the latter impossible. For example, Aristotle endeavoured to support the old circular astronomy by deductive inferences, one of which is as follows. He supposed, without proof, that stars are divine and eternal, and must have eternal motion. He supposed, again without proof, that the only eternal motion is circular, always returning on itself without terminus. From these two hypothetical premisses he deduced that the motion of the stars, being eternal, must be circular. No better example can be found of the abuse of the deductive method when applied too directly to concrete Nature. It is a case of two hypothetical premisses leading to a false conclusion. Every step is false. There is nothing for it but experience. The real question is how the stars move in point of fact; and when the question began to be rightly put, and the observations of Mars by Tycho Brahe and Kepler contributed the necessary experience, it was soon found to be the fact that the planets at all events do not move in circles or round the earth at all, but in elliptical figures and round the sun.

2) The second method is the empirical, which consists mainly of experience and induction. It is the Baconian method. Bacon saw the complexity of Nature and its subtlety far surpassing the subtlety of our sense and intellect. He concluded that the method of discovering 


\section{AS A MENTAL OPERATION}

the uniformities of Nature is not the deductive method of descending at once from principles easily found, but the inductive method of gradually ascending to principles. What Aristotle did for the deductive method of the mathematician Bacon did for the inductive method of the physicist. Aristotle, it is true, had recognized experience and induction. But what is due to Bacon is the discrimination between the ordinary and the scientific use of these mental operations. While ordinary men content themselves with isolated experiences and inductions from a few instances, the scientific man requires system in both. He must, in the first place, use experiment as well as observation, because Nature yields her secrets when tortured and put into artificial conditions by man. He must make his experience quantitative. His experience must be registered in writing, because it consists of too many facts for mere memory. It must be communicated to others and be the result of associated labours, of a society, of a royal society. Secondly, as he passes from experience to induction, the man of science must tabulate or arrange his instances according as they are instances where the thing investigated is present, instances where it is absent in similar circumstances, instances where it varies in degree. When his instances have been tabulated in these three ways, he must first use elimination, by excluding anything which is absent when the thing investigated is present, anything which is present when it is absent, anything which does not vary concomitantly with it. Then, at last, he will reach the affirmative induction that whatever is constantly present, absent, and varying with the thing investigated is always essential to this thing. This systematic induction from systematic experience is what 
Bacon justly called the new instrument-the Novum Organim. He was its discoverer. Baconian induction is founded on the sound principle that things which in experience are always present, absent, and varying together, are universally connected. It is a principle which is morally certain, without pretending to be mathematically necessary. Once recognized, it passed into modern science and logic. Accordingly, we can trace Bacon's three tables of presence, absence, and comparison, founded on it, through the writings of Boyle, Keill, and Herschel, till finally they became the essence of Mill's logic of induction under the titles of agreement, difference, and concomitant variation ${ }^{1}$.

The power of the empirical method can readily be exemplified before as well as after the time of Bacon: Astronomy, of all sciences, requires registered experience of many observations in series and periods. Bacon had this precedent before him, and especially the observations of Tycho Brahe on the planet Mars. He knew also of the discoveries of Galileo, first announced by the discoverer in the Sidereus Nuncius ( 6 10), and peculiarly valuable as showing that experience and induction do not require hypothesis. Having constructed his own telescope, and directed it to the stars, Galileo found (I) the roughness of the moon, so different from the smooth sphere ordinarily supposed; (2) the stars of the Milky Way; (3) the stars of which nebulae consist; and (4) most wonderful of all, the satellites of Jupiter, of which he made a regular nightly register. Later, with the same instrument, he discovered Saturn's ring and

1 See Bacon, Novum Organum, ii, ro-20; Boyle, Experiments about the Mechanical Origine of divers particular Qualities. Advertisements; Keill, Introductio ad veram Physicam, Lect. viii, Ax. 7 ; Herschel, Preliminary Discourse on the Study of Natural Philosophy, § I 45; Mill's Logic, Book iii, chap. 8. 
the phases of Venus, solar spots, and finally the moon's librations. But he was no mere observer, and made experiments as well as observations on gravity. Before the close of the sixteenth century he dropped bodies of different weights from the Tower of Pisa, and found that they did not fall, as had been supposed by the Aristotelians, with velocities in proportion to their weights, but, as Bacon learnt from him, with velocities nearly uniform, though not quite so on account of the resistance of the air which opposes greater delay to lighter bodies. Many years afterwards, after the death of Bacon, he followed this discovery of the uniformity of Qgravity by that of its uniform acceleration. The latter discovery was, like the former, experimental. Galileo had smooth inclined planes made; and then, by rolling balls down them and measuring the times and spaces of descent, he discovered inductively that the space fallen is always as the square of the time of falling; so that, if a body in one second of time falls about 16 feet, in two seconds it will have fallen 64 feet, four times as far (time 2-squared), in three seconds 144 feet, nine times as far (time 3 -squared). Nothing could better illustrate the power of the empirical method than these quantitative experiments of Galileo. They may enable us to dispense with later examples, such as Newton's experimental discovery of the dispersion of light and the numerous experimental analyses of modern chemistry. Finally, Galileo's employment of the empirical method, even before the appearance of Bacon's Novum Organum, does not detract from the originality of the latter. Galileo used method by genius; but Bacon] made the first logical theory of the empirical method, and has enabled many ordinary men almost to riva Galileo by showing them how to use its rules. 
But like mere mathematical deduction, mere physical induction has its limits. The former breaks down on the subtlety of Nature, the latter on its imperceptibility. As we pass from the simple to the complex there are too many alternatives for us to deduce one; and the further we proceed from experience beyond it the more difficult is it to get instances to induce universals. Curiously enough the nature of heat, the very thing which Bacon took to illustrate his inductive method, is precisely a case in point : it is beyond induction. Induction will reveal external causes of heat, such as conduction, convection, friction, percussion. It will reveal the external effects of heat: Bacon himself made experiments with the air and water thermometer, and showed how air when heated expanded and depressed the water. But he did not in any of his instances show what heat is, because none of them contained that imperceptible oscillating motion of particles which, as Bacon knew but not by induction, is essentially the nature or essence of heat. Something more then is wanted. By the empirical method we know what are called the 'phenomena' of heat. Now these phenomena are similar to those which we otherwise know to be consequences of motion. By combining these two kinds of knowledge we concludé that, similarly, the phenomena of heat are consequences of heat being a mode of motion. In other words, we infer the nature of heat, not by induction, but by that kind of deduction which combines 'phenomena' with 'laws'.

Deduction, when based on scientific induction, has several superiorities over its basis. "We have already shown that it is more definite, discovering as it does particulars beyond experience which are inaccessible to the generalities of induction. WSecondly, we have 
just mentioned its combining power. By bringing inductive facts under principles it enables us to reach further than induction, as in the case of the nature of heat. ${ }^{\text {*) }}$ Thirdly, it gives us a greater power of discovering causation beyond experience, and that in two ways. Sometimes we start from the effect and deduce its cause, sometimes from the cause and deduce its effect : that is, deduction is either analytic or synthetic. Sometimes, again, it may consist wholly of facts without causes. In short, deduction is full of variety. But, as we are now about to treat it, it is no longer the simple deduction of pure mathematics, but is interlaced with physical experience and induction in such a way as to produce a mixed method.

The superiority of mixed method comes out conspicuously in mixed mathematics, where deduction has more empirical materials than in the pure mathematics of numbers and figures. For example, Galileo, as we have seen, had a genius for observation and experiment. But no amount of experience and induction could tell him that a projectile moves in a parabola, because the curve in which it moves is beyond experience. How then did he make this discovery? By combining the empirical and deductive methods. He had induced that a body falls in a straight line by gravity with a uniform acceleration, so that the space is as the time squared. He had experienced that a projectile is emitted in a straight line, with what he concluded to be a uniform velocity as the time. But this empirical knowledge could not tell him in what line a projectile moves under the combined action of both forces. He knew, however, by the composition of forces, and by the Greek laws of conic sections, that a body having a uniform velocity as the time, and a uniform acceleration as the time squared, 
moves in a parabola, whose ordinate is as the tangent squared. He therefore deduced that a projectile is such a body moving in a parabola. This is a synthetic deduction from cause to effect, from the forces acting on a projectile to its consequent motion, when this motion could not have been discovered empirically. Again, Kepler's discovery that Mars moves in an ellipse round the Sun in one of the foci, and so that the radius vector from planet to sun describes equal areas in equal times, is a deduction; but in this case not causal, but a pure deduction of simple facts. The observations of the planet Mars, bequeathed by Tycho, and continued by Kepler himself, gave him a great number of positions successively occupied by Mars as they appeared. But the confused dance of a planet as it appears to a spectator from the earth could not by itself discover to him how it moves in relation to the sun. He had to consider in what curve a body occupying such positions moves; and this he could only do by the Greek laws of conic sections, from which he deduced that it moves in an ellipse, so that the radius vector to the sun in a focus describes equal areas in equal times. As Laplace says in his System of the World, "Without the speculations of the Greeks on the curves formed from the section of a cone by a plane, these beautiful laws might have been still unknown.' Kepler thus discovered that Mars occupies positions such that it moves in an ellipse, not as Mill thought, by a mere colligation of facts, for the facts did not contain the curve round the sun; nor, as Whewell thought, by induction, for the curve is not a generalization from the facts; but by deduction in the following form :-

The orbit of Mars has certain positions (by observation). 
Such positions are the properties of an ellipse (by conic sections).

$\therefore$ the orbit of Mars is an ellipse.

The most important, however, of all these kinds of physico-mathematical deductions is that from facts to their causes, because man knows more facts than causes, which are, however, the ideal of knowledge and yet are frequently hidden from experience. Newton's Principia is a conspicuous instance of this method. The full title, Philosophiae Naturalis Principia Mathematica, implies a combination of induction and deduction. It is also a combination of analysis and synthesis: it proceeds from facts to causes as well as from causes to facts. It contains, as everybody knows, the discovery that the planets gravitate to the sun with a force varying inversely to the square of their distance from it, and that all particles of ponderable bodies so gravitate to one another by the same law. But nobody ever experienced a planet gravitating to the sun. How then did Newton make the discovery? Let us go to the Principia for our answer.

Newton begins with the three axioms or laws of motion; the law of inertia of rest and motion, the law of proportion of force to effect, and the law of reciprocity, or equal action and reaction. He gives inductive evidence of these three laws. Though we know of no body moving altogether unimpeded, yet there are in our experience many instances of bodies moving straight forward the more freely the less they are impeded, which enable us to induce the law of inertia in the form that every body perseveres in its state of rest or motion so far as it is not affected by an impressed force. Secondly, the proportion of force to effect is obvious to experience. The third law had been already induced 
for the force of impact by Sir Christopher Wren's experiments before the Royal Society, which proved that, if one body impinge upon another, and by its force change the motion of the other, that first body also, because of the equality of the mutual pressure, will undergo an equal change in its own motion towards the contrary part. Newton repeats Wren's experiments, and, having added fresh instances from magnetism and other attractions, extended the law from impact to all forces, and induced it in the universal form, action and reaction are always equal and opposite. Thus did Newton finally establish the main principles of dynamical mechanics by the empirical method of Bacon. But he went beyond induction, which never could have discovered the imperceptible link between the sun and its planets. The sequel is deduction; and even under the head of Axioms he immediately uses the third law, as induced from impact, to deduce that the whole momentum of bodies acting reciprocally on one another, together with the state of their centre of gravity, is conserved. So true is it that ${ }^{-}$induction leading to deduction is the most fruitful method of natural science.

In the first book of the Principia Newton, starting from the general laws of motion, proceeds to deduce a great number of derivative laws in dynamical mechanics. Two of these laws require special notice here: the first, that every body, which moves in a curve, and by a radius drawn to a point describes about that point areas proportional to the times, is urged by a centripetal force to that point ; and the second, that the centripetal forces of bodies, which by equable motion describe different circles, are to one another as the squares of the arcs described in equal times applied to the radii of the circles; while by a further corollary, if the squares of the 
periodic times are as the cubes of the radii, the centripetal forces of the bodies will be inversely as the squares of the radii (i.e. the distances from the centre); and vice versa. Further, he deduces these laws for all kinds of curves.

The derivative laws of dynamical mechanics thus deduced from the laws of motion served Newton in their turn as his major premisses for the deduction of the cause of planetary motions in ellipses round the sun. But first he wanted these facts themselves as his minor premisses. He derives them from Kepler's laws, which he calls Phaenomena. They are three: planets move in ellipses round the sun in one of the foci; they describe, by the radii drawn, not to the earth, but to the sun, areas proportional to the times of description; and the squares of their periodic times are as the cubes of their mean distances from the sun. Starting then from mechanical laws in the major, and from astronomical phenomena in the minor, Newton was ready to deduce the real cause of planetary motions in ellipses round the sun; namely, their centripetal force, or gravitation to the sun.

His deductions, which are formal analytic syllogisms from fact to cause, are as follows :-

(I) Every body which moves in a curve, and by a radius drawn to a point describes about that point areas proportional to the times, is urged by a centripetal force to that point (Principia, Book I, Prop. 2).

The planets by the radii, drawn, not to the earth, but to the sun, describe areas proportional to the times of description (Ibid., Book III, Phenomenon 5: Kepler's Second Law).

Therefore, the planets are urged by a centripetal force to the sun (Ibid., Book III, Prop. 2). 
(2) When the squares of the times are as the cubes of the radii, i. e. the distances, the centripetal forces of the bodies will be inversely as the squares of the radii, i.e. the distances (Principia, Book I, Prop. 4, Cor. 6).

The squares of the periodic times of the planets are as the cubes of their mean distances from the sun ${ }^{1}$ (Ibid., Book III, Phen. 4 : Kepler's Third Law).

Therefore, the centripetal forces of the planets tending to the sun are inversely as the squares of their distances from the sun (Ibid., Book III, Prop. 2).

Thus one of the greatest discoveries of all time, the application of mechanics to astronomy, the identification of the laws of motion and gravitation in the sublunary and stellar worlds, the revelation of the real cause which makes the planets revolve, not round the earth, but round the sun, the final confirmation of the Copernican system and Kepler's Laws by explaining them, was found from experience and after induction, but beyond both these narrower operations by the comprehensive power of analytic deduction from the facts of planetary motion to their cause, centripetal force tending to the sun. Moreover, this deduction was essentially syllogistic, from a mechanical major and an astronomical minor to an astronomical conclusion, by a method fundamentally Aristotelian. It was a 'syllogism of fact', as Aristotle would call it; that is, not from cause to effect, but from fact to cause. It was an analytic syllogism, in which the fact is in astronomy, the cause in mathematical mechanics; and Aristotle, who had already ${ }^{2}$ made this distinction, saw that the fact may be in physics and the cause in mathematics, many centuries before Newton entitled his book

1 For example, as compared with the earth, Jupiter's time squared, $12^{2}$, is nearly equal to his distance cubed, $5^{\frac{1}{4}}{ }^{3}$, from the sun.

${ }^{2}$ Aristotle, Posterior Analytics, i. 13. 
Philosophiae Naturalis Principia Mathematica. So far Newton justified the deductive logic of Aristotle, and so far he transcended the inductive logic of Bacon. He found that induction is not the sole avenue to all the uniformities of Nature, because from inductive principles deduction goes on to derivative uniformities. At the same time, Newton's example did not prove Aristotle wholly right, or Bacon wholly wrong. His method goes beyond both. Having further deduced that the satellites of planets and the moon in relation to the earth revolve by the same centripetal force of gravitation, he returned from deduction to induction. Using earthly bodies, moon, planets, and satellites as great groups of instances, he induced the universal law of gravitation, and that all the particles of ponderable matter gravitate to one another by the same law. This is exactly a Baconian induction of a uniform principle from heterogeneous instances, from instances however gained not by experience, but by deduction. But, in fact, the scientific method of Newton's Principia, viewed as a whole, is neither the deductive Aristotelian, nor the inductive Baconian, but both : it is the interaction of induction and deduction in a mixed method.

The method of analytical deduction from facts to causes, and the method of first describing facts and then explaining them by their causes ${ }^{1}$, had been anticipated by Aristotle, who, however, somewhat lost the fruit of his wisdom by too great an emphasis on synthetic mathematical demonstration. It remained for Mill in our times to revive the explanatory method, which consists mainly of facts and causes, and the explanation of the former by the latter. Mill, however, did not understand analysis,

1 See De Partibus Animalium, Book I. 
the most potent kind of explanation. He takes Newton's explanation of Kepler's Laws by gravitation as an example. But he misunderstood Newton's method, by describing it as if it explained the facts of planetary motion by making a hypothesis of gravitation as cause and then deducing from it the facts. Now, Newton may have made a hypothesis beforehand according to the story of the apple. But hypothesis of a cause and hypothetical deduction of facts are not the method of Newton's Principia, which makes no hypothesis of gravitation, and does not use gravity to the sun as cause to deduce planetary motions as facts, but, on the contrary, uses planetary motions as facts to deduce gravity to the sun as their cause. The explanatory method of Newton is neither hypothetical nor synthetic, but scientific and analytic.

Mill failed to discriminate between analytic and synthetic deduction, and as a consequence did not perceive that frequently in the history of science the original order of discovery is analytic and the order of teaching synthetic. Thus, when once Newton had used planetary motions to deduce gravitation, gravitation was then used to deduce planetary motions, inversely. But from imperfect appreciation of the extraordinary power of deduction, Mill misunderstood analysis and synthesis, as well as their connexion: On the one hand he substitutes hypothetical deduction for analysis. On the other hand, missing the analytic foundation of synthesis, he represents synthetic deduction as also hypothetical. He has had immense influence even on his opponents. The result is that, since the publication of his Logic, hypothesis has been ridden to death; and many scientific inferences are misrepresented as mere hypothesis to explain facts, which are really perfectly scientific deduc- 
tions either from facts to causes, or from causes to facts, or first one and then the other, because analysis is the ground of synthesis. The truth is that the explanatory method is various, being sometimes from facts to causes, and sometimes from causes to facts. Sometimes, again, the premisses are hypothetical ; but sometimes they are sound principles, as in Newton's Principia.

Besides the mathematical, the empirical, and the explanatory, Mill has yet a fourth method, the verificatory method, which consists of principles, or sometimes hypotheses, followed by deductions and verifications. It is another species of mixed induction and deduction, and a famous example is supplied by the discovery of the planet Neptune from the deflection of the planet Uranus from its orbit round the sun. Herschel in I 78I had found Uranus empirically by observing it through the telescope, and at least two of its satellites. But Neptune was afterwards discovered deductively. The law of gravitation, thanks to Newton, had become a principle so firmly established that it could be used to explain the deflections of planets from their orbits by their gravitation to one another. Thus some of the deflections of Uranus were explained by its gravitation to Jupiter and Saturn. But there remained other deflections on this or that side of its orbit. It was therefore inferred that, as deflections are caused by gravitation in the direction of deflection, and as Uranus deflects in a given direction, it must be gravitating to an unknown planet in the direction of deflection. So far deduction. Then came verification. Adams and Leverrier, having each calculated the direction, the planet, since called Neptune, was actually observed through the telescope in that direction. Thus deduction was verified empirically.

When we consider our liability to error both in 
original inductions and in subsequent deductions, and add our tendency to begin our deductions with hypotheses in want of principles, we realize the importance of verifying our conclusions. A curious example is afforded by the present condition of the theory of Free Trade, which Mill regarded as discovered and proved by the verificatory method. Adam Smith had begun with principles of human nature, such as that every man is a better judge of his own interest than any legislator can be for him, and had deduced a conclusion in favour of as much liberty as is consistent with right. The ultimate result was the partial adoption of Free Trade. Now Mill regarded this conclusion as verified by the prosperity of nations which had adopted this system. But unexpectedly, since his time, some nations which have remained protectionist have risen to great prosperity, especially in America and in Germany. What seemed proved has become matter of doubt and dispute. Not that Smith's conclusion has been proved false. Theoretically, it was a general presumption in favour of liberty from restrictions, deduced from premisses immersed in the complex matter of human affairs, and applicable only according to circumstances. In passing into practice, it became an absolute dogma, the exaggerations of which cannot be verified. The problem should be referred back from politicians to philosophers, in order to distinguish the limits within which the theory of Free Trade is applicable, and capable of being verified by historical facts without exception.

We have reviewed some of the methods of science. It appears that pure mathematical and pure empirical methods are clearly defined but limited. Mixed method, on the other hand, is far-reaching and complicated. The part of the logician is to recognize its various ramifi- 
cations. Sometimes it is induction and deduction of facts, and sometimes also of causes. Sometimes it is deduction from facts to causes, and sometimes deduction from causes to facts. Sometimes it is hypothetical, but not so often as is nowadays believed. The facts, again, may precede the hypothesis advanced to explain them; or the hypothesis may be advanced to deduce consequences to be afterwards verified. It may be remarked also that there is a consilience of induction and deduction; and that of two kinds. We may find a fact empirically and then deduce the same fact; or we may deduce it and then find it empirically to be a fact. As Aristotle said, reason seems to confirm phenomena, and phenomena reasoning. Moreover, as he also said, the very power of deducing many consequences from a premiss confirms it by a kind of reaction. Everything proves that induction and deduction are inextricably. interlaced in science: witness the conspicuous example of Newton first inducing 'axioms', next deducing conclusions from, but beyond experience, and then using those conclusions as though they were particular instances to induce the universal gravitation of ponderable bodies.

So various and subtle is this mixed method that it is difficult to classify it. It can hardly be confined within Mill's explanatory and verificatory methods, at any rate in the narrow sphere within which he restricted the former, and with the hypothetical turn which he gave to both methods. But are not explanation and verification, even at their widest extent, merely varieties of the mixed method of induction and deduction, always interacting and also confirming one another in many intricate ways? In conclusion, the lesson of all scientific method, and especially of mixed method, is this : start 
from experience and systematic experience; transcend experience by inference and the generalizing power of reason; but return to experience for fresh data to induce and deduce; in order to discover truth, which is the coincidence of what we judge and say with the objects capable of being apprehended by sense and reason united $^{1}$; in order to construct works, as monuments of the reign of man over nature; in order, above all, to act for the general good, and the reign of man over himself.

1 It has become a fashion to doubt the possibility of discovering the truth of things, on the Kantian ground that we cannot compare things known with things beyond knowledge. But truth is not in this parlous condition: it only requires us to compare our judgements with things known-a condition practicable enough. Sense and reason are the origin of our knowledge of things within and without; we constantly compare our judgements with what we discover by these faculties of apprehending reality; and there is an appeal from judgement to the things which become objects of sentient and rational knowledge, though without appeal, and without the necessity of it, to another set of things, ex hypothesi unknown, beyond. Truth, therefore, is not an impracticable agreement of knowledge with things beyond, but the actual coincidence of judgements and propositions with things capable of being apprehended by sense and reason united in knowledge and especially in scientific knowledge acquired by scientific methods. 


\section{II}

\section{ON SOME ASPECTS OF THE SCIENTIFIC METHOD}

(Professor F. Gotch, F.R.S.)

ThE scientific lectures of the Summer Meeting for this year have been arranged upon lines which differ essentially from those of preceding gatherings. They are all dominated by one leading idea, and have in consequence a unity of purpose which gives a special interest to each member of the series; this idea is to set forth the operation of the scientific method as exemplified in the scientific field of human knowledge and achievement. "Strictly speaking, the scientific method is a phase of rational thought applied more particularly to the phenomena of nature, and, in the first instance, to these only, but its scope is in reality more comprehensive, and it would be possible to bring within its operation the greater part of the subject-matter to be treated at this meeting. Upon various grounds its exemplification has been limited to certain specified portions of knowledge and research, chiefly in natural science.

Lectures in Physics, Chemistry, Biology, Physiology, and Psychology will set forth the value of the method in connexion with experimental science; others in Astronomy, Geology, Anthropology, Archaeology, and History will show its use in dealing with observed or recorded facts, and in framing from these scientific generalizations. 
The novelty and the somewhat ambitious character of the scheme rendered it desirable that it should be introduced by lectures of a general nature ; the scheme, being a nativity, should, it was urged, be vouched for by sponsors. The duties of sponsorship are responsible as well as honourable; and whilst acknowledging the honour of being selected to aid in discharging them, I am but too conscious of the responsibility. I feel, however, fortunate in that the most important part of these duties has been placed in other and abler hands; my friend and colleague, the President of Corpus, who has introduced the whole subject as an operation of the mind, has done so in such a lucid and instructive manner as to relieve me of much anxiety in this respect. Indeed, since the scientific method, whatever it may not be, is undoubtedly a mode of thought, it is open to question whether there is room for any further general introduction of the subject. This consideration has, I confess, somewhat embarrassed me, and other circumstances have contributed to my embarrassment; the necessity of treating the matter generally, and yet of bringing forward particulars to give the treatment interest and point, the extensive scope of the method even when limited to natural science, the difficulty of rendering myself intelligible in a subject which has so many side issues, and the difficulty of selecting an interesting line of treatment; above all, the difficulty of prefacing any line of treatment with some definite indication as to what this phrase, 'the scientific method', means to me.

One may be a scientific man, pursuing scientific investigations, and making from time to time definite contributions to scientific knowledge, without being able to answer shortly the question, What do you understand by this phrase, 'the scientific method'? How can it be 
expected that a few apt sentences should describe definitely the processes by which man achieves his destiny and slowly rises to a knowledge of his surroundings? At the risk, however, of being thought dogmatic, I venture to state, in one sentence, what, as a follower of Bacon, I believe to be the essence of my subject; the sentence which appeals to me as descriptive is, however, not Bacon's but Virgil's :-

Felix qui potuit rerum cognoscere causas ${ }^{1}$.

In this well-known and felicitous line I seem to grasp the core of my subject, for this describes the scientific spirit, happy only in so far as it strives to know the causes of things. The scientific method comprises all those modes of mental activity which are best adapted to attain this end. 'It is not a hard-and-fast system of thought; it is not a particular method of observation or of experiment; it is not any special technique. In itself, the method is meaningless; it acquires merit through its aim, and is significant because of its purpose. Its form may, and indeed must, be plastic, varying with the condition of man and of nature, but its end remains throughout the, same-the revelation of the truth about things." Man is so constituted that he must strive to understand himself; his experience has to be made more intelligible to him; in natural science this intelligibility is bound up with the causation of those natural phenomena which surround him from the cradle to the grave. I use the term 'causation' here in the sense so definitely indicated by Mill, 'a cause which is itself a phenomenon without reference to the ultimate cause of anything,' what, in short, is spoken of in metaphysics as the 'physical', not the 'efficient' cause; the term is thus employed 'in that

1 Virgil, Georgics, ii. 490. 
sense alone in which one physical fact is said to be the cause of another'. ${ }^{1}$

The mere sensory recognition of any phenomenon does not constitute science; science is the causative arrangement of phenomena, and the recognition is but the first step towards its achievement. Science is thus an intellectual construction, the creation of man's reason, the point of view of those who strive to set all experience in this causative array. This particular point of view, handed down from one generation to another in scientific literature, is being continually modified in detail and enlarged in scope to meet the mental needs of those who feel this intellectual craving for intelligible explanation; but, however modified or enlarged, it is always directed towards the same goal, that of satisfying intelligent curiosity, of answering the question, Why?

The scientific method is only an implement for this travail of the scientific spirit, an implement with which mankind labours to erect a dwelling in which it may rest satisfied. What matter though the limitations of man's powers are such that this dwelling can never be completed? What matter though man realizes that he is constructing an insecure, and, from the nature of things, a merely temporary shelter? For the moment it is such a shelter, and, pausing under it, he renews his strength and plans how to give it a more permanent character and make its foundations more stable.

This spirit was present in the great men of antiquity; it languished during the middle ages, but was reincarnated in the fifteenth and sixteenth centuries, and, quickening in the seventeenth century, has since that time never languished. From this period we date the scientific method as it exists to-day.

${ }^{1}$ Mill, System of Logic, Book III, chapter v. 
Is it possible to give general expression to the prominent features which give the method its peculiar potency? And if so, what line of treatment would be most suitable for the purpose?

A convincing line, but one necessitating special knowledge on the part of my audience, would be to select groups of scientific problems and attempt to show how they have been investigated. I feel that I must leave this to the eminent scientific men who are to deal with such problems later.

After much consideration, I have decided to adopt a line of treatment which may, at least, have the charm of novelty. The rise and development of any new method which transforms human thought, is always subjected to the fierce fire of hostile criticism; and it may be fairly assumed that the grounds of such attacks involve the more prominent characteristics of the new departure in thought. Bearing this in mind, we may, I think, arrive at the general features of the scientific method by an examination of some of the grounds upon which it was assailed in the seventeenth and eighteenth centuries. The assault has continued on more or less the same lines to the present day, indicating that the features selected for attack were not extrinsic ones belonging to the period, but are intrinsic to the method itself; they constitute, in short, some of its more essential characteristics.

These characteristics are displayed in the attacks made upon the early work of the Royal Society, which was founded in the seventeenth century. The birth of this celebrated society is a landmark in the history of the scientific method. One of the first-fruits of the reincarnation of the true scientific spirit was the rapid growth of an enthusiastic interest in the study of nature. Drawn together by similar interests and intellectual desires, men 
formed themselves into groups for pursuing this study, and among such groups two were conspicuous. One of these was a small society meeting in Gresham College, London, the other an Oxford society which met from I649 onwards in the lodgings first of Dr. Petty of Christ Church, and later in those of Dr. Wilkins, Warden of Wadham College. ${ }^{1}$ These two private societies were in active correspondence, and constituted together what Robert Boyle termed 'the Invisible College'; from these sprang in I66I the Royal Society, the object of which, as set forth by its original members, was as follows: The Society was to be a collection of 'divers worthy persons inquisitive into natural philosophy and other parts of human learning, and particularly of what hath been called the New Philosophy or Experimental Philosophy, which, from the times of Galileo at Florence and Sir Francis Bacon in England, hath been much cultivated in Italy, France, Germany, and other parts abroad as well as with us in England'.

At the meetings of the Society discussions were to be held on a large variety of the above subjects, and demonstrations were to be given of natural phenomena, with special reference to their causation. The New Philosophy had for its aim the expression of causation and its instrument was the scientific method.

The Royal Society, with this aim and method, was regarded at first as a mere eccentricity, but soon it was

${ }^{1}$ Dr. Petty, afterwards Sir William Petty, is referred to by Wallis who wrote $A$ Defence of the Royal Society, published in 1678 . In the account given by Wallis of the 'first ground and foundation of the Royal Society' it is stated that the Oxford Society comprised Dr. Wilkins, Dr. Wallis, Dr. Goddard, Dr. Ward, Dr. Bathurst, Dr. Petty, Dr. Willis and divers others, who 'brought these studies into fashion there ; meeting first at Dr. Petty's lodgings (in an apothecarie's house) because of the convenience of inspecting drugs, and the like'. 
fiercely attacked, and it is possible to distinguish in the character of these attacks the special features of the Society's work which were assailed; these I now propose to consider under different heads.

\section{The freedom and sceptical character of the experimental} inquiry into Nature.

The whole realm of Nature was regarded as lying open to investigation, and all its phenomena were considered as fit subjects for scientific inquiry; but to most men of that time Nature was something to be accepted, not inquired into. It was felt to be indecent, almost immoral, to probe the secrets of what many men still regarded as a glorified human being. What right had any one to enter Nature's private dwelling and ruthlessly examine the character and arrangement of her furniture, or the rules which governed her domestic economy.

That Nature was commonly regarded in this anthromorphic light and even endowed with such human attributes as reason, emotion, volition, and morality, is shown by an examination of Boyle's Free inquiry into the vulgar notion of Nature. He states that by the term Nature 'was commonly meant a semi-deity or other strange kind of being'. In support of this he cites the chief axioms as to Nature which were current in his day. 'Nature is exceedingly wise and all her works are performed with understanding. Nature does nothing in vain. Nature never fails of her purpose. Nature always does what is best. Nature always acts in the shortest manner. Nature is never too lavish, never too sparing. Nature always preserves herself. Nature always watches to preserve the universe.' In addition to all these it appeared that she was susceptible to the emotion of fear. This was incorporated in the familiar axiom, 'Nature 
dreads a vacuum.' It was gravely asserted that, owing to this great and exceeding fear, she caused water to ascend in pumps; at all hazards a vacuum must be prevented, and Nature was ever on the watch to stop its occurrence, for if it did occur it would be an indescribable shock to her feelings.

The New Philosophy would have none of this; it displayed no regard to Nature's feelings; it did not enter the minds of its exponents that it was immoral to examine all her works; it actually defined her as 'the aggregate of all the bodies that make up the world'. To many it appeared that this last proceeding was the cruellest blow of all; it destroyed the personality of Nature; this disappeared sacrificed on the altar of the scientific method.

It was easy to show that a new philosophy which started so destructively was the invention of the devil, a weapon forged by him for the wreck of mankind; and this view was strengthened by the circumstance that the scientific method, which involved an investigation into all natural phenomena, advanced cautiously; for its exponents, realizing the errors of preceding centuries, enjoined upon those who used it a sceptical attitude of mind. 'I expect,' says Boyle in the preface to his philosophical works, 'it will be observed to my disadvantage that I speak doubtfully upon most occasions, which seems to argue a diffidence of the opinions I incline to, but having met with many things for which I could assign no probable cause, and with some for which many very different ones might be alleged, I dare speak positively and confidently of very few things except of matters of fact.'

This sceptical attitude provoked hostility from two sources. It was felt to be a dangerous habit of mind to 
speak doubtfully; if of nature then why not of other matters. It was felt to be humiliating that man with his intellectual powers should have to speak doubtfully; these scientific men, when pressed, are sure of nothing, not even of those things of which we are most firmly convinced, those which we can see, handle, and hear.

The hostility displayed in the seventeenth century to the freedom and sceptical character of scientific inquiry into Nature has diminished in intensity with educational advance, but it still persists. Even at the present time scientific investigation is considered as needing to be circumscribed, and its extension in certain fields of natural phenomena is regarded as immoral. Scientific men are still ridiculed because they refuse to give a categorical answer of the yes or no type, the sort of answer which the so-called practical man generally demands to his plain question. But in the words of an old writer, 'true knowledge is modest and wary, 'tis ignorance that is so bold and presuming ${ }^{1}$.'

The recognition of reason as the only ultimate authority.

The aim of the New Philosophy was to create an intelligible scheme of natural phenomena, these being set in orderly array from the point of view of their sequence in time and of their causative connexions. Every one of the presumed relationships between phenomena was thus brought before the bar of reason, and judgement was delivered upon it by this authority. With alarm even those who were themselves intellectual recognized that this newly set up authority of reason endangered that of tradition. There is no question that their fears were justified, for every scientific writer of the

${ }^{1}$ Glanville, The Vanity of Dogmatising. 
period casts off the shackles of such speculations into Nature as derived their sole authority from this source. 'But the mortallest enemy unto knowledge,' wrote Sir Thomas Browne in 1646 , ' and that which hath done the greatest execution upon truth hath been a peremptory adhesion unto authority and more especially the establishing of our belief upon the dictates of antiquity.'

The search after a causative explanation of natural phenomena was, therefore, represented by its opponents as hostile to the authority of the State and subversive of the Christian faith ; its pursuit must, it was said, inevitably lead to the extinction of the Universities, whose business it was to teach the wisdom of the past, and which, according to Sir Thomas Browne, were at this period 'though full of men oftentimes empty of learning'.

Tracts were written fulminating against the Royal Society, which was rightly regarded as the head quarters of the New Philosophy; attacks and rejoinders were as thick as leaves in June. Sprat found it desirable to write a history of the foundation and work of the Society in order to demonstrate that it did not exist for the purpose of upsetting Church and State, but that when fully understood the New Philosophy would be found to be a bulwark of Christianity, not its destroyer. In an article upon the Royal Society, included in the Quarrels of Authors, the elder Disraeli gives an interesting account of this literary controversy. From this it appears that the zeal of the opponents often outran their discretion; for not only the aims but many of the obvious practical results of scientific inquiry were inveighed against. Crosse, the vicar of Chew Magna in Somersetshire, anathematized the Royal Society as a Jesuitical conspiracy against both society and religion; he regarded the use of the newlyinvented optick glasses as immoral, since they perverted 
the natural sight and made all things appear in an unnatural and, therefore, false light. It was easy, he said, to prove the deceitful and pernicious character of spectacles; for take two different pairs of spectacle glasses and use them both at the same time, you will not see so well as with one singly; therefore your microscopes and telescopes, which have more than one glass, are impostors. Hostility went further than this; it was declared to be sinful to assist the eyes, which were adapted to the capacity of the individual, whether good, bad, or indifferent. It was argued that society at large would become demoralized by the use of spectacles; they would give one man an unfair advantage over his fellow, and every man an unfair advantage over every woman, who could not be expected, on aesthetic and intellectual grounds, to adopt the practice.

That it was the newly-erected tribunal of reason which was the mainspring of all this opposition is clear from the circumstance that the hostility proceeded not only from the extreme advocates of traditional authority, but also from those who demanded the release of man's thought from all authority, including that of reason. Men of this stamp, 'in whose breasts the embers of the revolution were still hot,' were panic-struck at the rapid advance of the New Philosophy, and imagined 'that the advocates of Popery and arbitrary power were returning upon them disguised as natural philosophers'. In this fear they were strengthened by the exclusive and, to them, consequently, sinister character of the deliberations of the Royal Society, and by the term used by Boyle to describe its members, 'the invisible college.' Advantage was taken of these fears by writers of repute; thus Stubbe, a physician at Warwick, wrote a pamphlet entitled 'Campanella revived, or an Enquiry into the 
History of the Royal Society; whether the Virtuosi there do not pursue the projects of Campanella for reducing England into Popery'.

The hostility to that authority of reason, which is the 'mainspring of the scientific method, has continued down to the present day. It was shown in the virulence with which natural selection and evolution were attacked; it displays itself in the peculiar temper of Christian scientists, and such other exponents of modern free thought, who regard with suspicion that appeal to reason which the scientific method demands.

\section{The particular character of scientific work.}

The enthusiasm of the scientific men of the seventeenth century made them inquire into the truth of current opinions, even those which were in themselves highly improbable. Impatient to sift the true from the false, some of the earlier scientific investigators lost their sobriety in the search for strange and extraordinary phenomena. 'There was,' says Disraeli, in the Miscellanies of Literature, 'an almost infantine simplicity in the work of some of the early members of the Royal Society.' 'In a memorial in Sprat's History, entitled 'Answers returned by Sir Philliberto Vernatti to certain inquiries sent by order of the 'Royal Society', are some

${ }^{1}$ Campanella, who was an Italian philosopher and a contemporary of Bruno, advocated the views of the universal vitalists. He considered the whole world, down to its simplest elements, to be capable of sensation and to be endowed with properties analogous to the consciousness of human beings. With these he combined extraordinarily restricted political views, for, although he desired to shake off the yoke of authority in philosophical matters, he strenuously upheld the necessity of establishing a universal autocracy of the most stringent type in all matters connected with conduct and social life. He spent almost his whole life in the prisons of the Inquisition. 
extraordinary questions and descriptions of nonentities. Question 8 ran as follows': 'What ground there may be for that relation concerning horns taking root and growing about Goa?' Sir Philliberto examined into the matter with due gravity, although from his reply it appeared that it was a coarse Portuguese jest characteristic of the age, and had reference to the morals of the inhabitants.

In these and like inquiries the Society made itself a target for the wits of the day to shoot at. It must be remembered in their defence that the current beliefs of even educated persons in regard to natural phenomena were often incredibly foolish; they were, however, so firmly held that rigorous logical proofs of their absurdity were necessary in order to dislodge them. In this respect I commend to your consideration that really amazing and most erudite compilation by Sir Thomas Browne, entitled Pseudodoxia Epidemica, or Enquiries into very many received tenets and commonly presumed truths. It is a delightful work, containing an enormous mass of fact and fancy, from which the author weaves elaborate arguments against the vulgar errors of the day. I select a very few of the errors cited by him in order to show the character of the current beliefs which he felt it necessary to examine into and to confute.

'That a loadstone held in the hand doth either cure or give great ease in the gout.'

'That a kingfisher hanged by the bill showeth in what quarter the wind is by an occult and secret property converting the breast to that point of the horizon from whence the wind doth blow.'

'That the diamond, which is the hardest of stones, not yielding unto emery or anything but its own powder is yet made soft or broken up by the blood of a goat.' 
- That there is a property in the Basil (plant) to propagate scorpions, and that by the smell of the plant they are bred in the brain of man.'

'That the elephant hath no joints, wherefore being unable to lie down he sleepeth against a tree, which the hunters observing do saw almost asunder, whereon the beast relying, by the fall of the tree falls down itself and is able to rise no more.'

Even in the present day it is astonishing how many current beliefs exist in regard to natural phenomena which have little or no foundation in fact; instances of such vulgar errors are familiar to us all.

The work of those scientific men who inquired into commonly-received opinions as to natural phenomena, although ridiculed, was in reality one aspect of the use of the scientific method, that, namely, which tests all things relating to nature.

But ridicule extended to other phases of scientific work, and particularly to biological study. ' There has,' says the author of the Miscellanies, 'always been a tendency for men of a meditative cast, so-called philosophers, to ridicule naturalists and their occupations.' The celebrated Dr. South, in his oration at Oxford, said, with reference to many members of the Royal Society of his day, 'they can admire nothing except fleas, lice, and themselves'. This contemptuous attitude of society is most divertingly displayed by Goldsmith in one of those inimitable letters which the Citizen of the World addresses to his friend Fum Hoan, first president of the Ceremonial Academy at Pekin :-

'I am amused, my dear Fum, with the labours of the learned here. One shall write you a whole folio on the dissection of a caterpillar. Another shall swell his works with a description of the plumage on the wing 
of a butterfly; a third shall see a little world on a peach leaf, and publish a book to describe what his readers might see more clearly in two minutes only by being furnished with eyes and a microscope. Yet, believe me, my friend, ridiculous as these men are to the world, they set up as objects of esteem for each other. They have particular places appointed for their meetings; in which one shows his cockle-shell and is praised by all the society, another produces his powder, makes some experiments that result in nothing, and comes off with admiration and applause; a third comes out with the important discovery of some new process in the skeleton of a mole, and is set down as the accurate and sensible; whilst one, still more fortunate than the rest, by pickling, potting, and preserving monsters, rises into unbounded reputation.'

'The labours of such men instead of being calculated to amuse the public are laid out only in diverting each other. The world becomes very little the better or wiser for knowing what is the peculiar food of an insect that is itself the food of another, which in its turn is eaten by a third; but there are men who have studied themselves into a habit of investigating and admiring such minutiae. To these such subjects are pleasing.'

There runs through the whole of this delicious passage that delicate humour which is so characteristic of Goldsmith's writings; as a satire, however, it is directed against the opinion of the town which the Citizen of the World is represented as describing. Goldsmith himself had some biological interests, as evidenced by his Familiar introduction to the study of Natural History and by his Animated Nature. ${ }^{1}$

${ }^{1}$ Goldsmith was, however, in no sense a naturalist; he was not even qualified to write about science, and his so-called scientific essays are 
It seemed to the wits of the day that the prolonged labour, necessitated by a detailed examination of the structure of minute natural objects, was childish and ridiculous; it seemed to society at large that it was ignoble and even contemptible; it seemed to practical men meaningless, much ado about nothing, labour of no practical utility to any living soul, and, therefore, worthless.

The ridicule extended to the experiments which formed such an important part of the New Philosophy; they were considered as laborious folly which could not possibly lead to any practical conclusion. In Gulliver's Voyage to Laputa, Swift has satirized the experimental work of the Royal Society. Gulliver is, you may remember, introduced into the learned academy of Lagado. There he finds the natural philosophers all busy with projects and honoured by the title of projectors. One had been for eight years engaged on a project for extracting sunbeams out of cucumbers, and placing them in hermetically-sealed vials in order to let them out in raw inclement summers. Other projects were calcining ice into gunpowder, softening marble into pillows and pincushions, petrifying the hoofs of a living horse to preserve them from foundering, whilst a famous project was that of a most ingenious architect for building houses by beginning at the roof and working downwards to the foundation.

The extent of the ridicule, satire, and invective poured upon scientific work is only faintly portrayed by the above samples; it will, however, be observed that out of many features presented by these attacks, I have

merely popularized excerpts; they contain ludicrous mistakes. Dr. Johnson said of him that 'if he can tell a horse from a cow, that is the extent of his knowledge of zoology'. 
selected three. First, the ridicule excited by the fact that detailed inquiry should be made into the truth of current beliefs and vulgar errors associated with natural phenomena. Secondly, the contemptuous feeling aroused by laborious work upon the minute characters of natural objects, work involving observations on materials regarded often as unpleasant and always as quite unimportant. Thirdly, the condemnation as ignoble trifling, and sheer waste of time and energy, of all scientific experiments which did not carry on their surface intelligible utility and which, being therefore pronounced as of no practical advantage to mankind, were satirized as eccentric folly.

These lampoons and satirical criticisms did not languish for want of reply, and one of the earliest serious rejoinders was that of the celebrated writer, Joseph Glanville, who matriculated at Exeter College, Oxford, in 1652. He became a Fellow of the Royal Society in I665, and had a wonderfully clear view of the range and profundity of the problems of science. In a work, entitled Plus ultra, or the progress and advancement of knowledge since the days of Aristotle, he contrasted the past with the age in which he wrote. This comparison brought out the obvious fact that however useless and absurd many of the investigations may seem to the generation in which they are undertaken, 'posterity has always found things which are at the start but rumours converted into practical realities.' It is, indeed, strange that the objection of inutility should survive, and still survives, in the face of the recurring demonstration that the very investigations once scoffed at as useless can, in the course of a few years, become highly honoured for their great value. Plus ultra was greatly appreciated by the Royal Society; Evelyn thought the argument conclusive, and exultingly exclaimed: 
'Now let the moon-dogs bark 'till their throats are dry.' 1 Glanville became actually prophetic in his survey. With the vision of a seer he anticipated 'that to confer at the distance of the Indies by sympathetic conveyances may be as usual to future times as to us in a literary correspondence.' His antagonist, Stubbe, covered him with ridicule for making such a preposterous statement.

The particular aspect of the scientific method which evoked all this criticism and ridicule was, and still is, a very real one. The goal towards which the scientific worker aspires is not increase of personal wealth-indeed, this is often diminished by his labour; his aim is to disclose scientific truth, and for this purpose every natural phenomenon, however minute or apparently useless, will suffice to kindle his enthusiasm and to employ all his faculties. His occupation is ennobled by its purpose, that of setting forth natural phenomena in causative array. To effect this purpose investigations have to be undertaken of the aspects which these phenomena present under all manner of conditions. It is instructive in this connexion to consider the significance of scientific experiment as contrasted with scientific observation. Science always begins with observation; many observed phenomena have occurred once for all time and cannot be observed again; others occur daily, hourly, when we please, and observations can then be repeated over and over again. As regards some of these, although the attendant conditions change, any alteration in such conditions is beyond our control, but in regard to a great mass of natural phenomena it lies within our power, not only to obtain their occurrence when we please, but also to alter the conditions under which such occurrence takes place; we can

${ }^{1}$ See Greenslet's Study of Joseph Glanville. 
now make fresh observations within a changed setting. This last procedure constitutes a genuine experiment ; by its means we are able to eliminate various antecedents, and to adjudicate upon their causal relationship to the observed phenomena. We thus approach more nearly to the attainment of the causative sequence, and for this reason experimental research is justly held as an invaluable technique; it constitutes the most potent of all the methods which are employed for the furtherance of scientific knowledge. Indeed, it frequently happens that no real advance is made as long as some definite procedure of this type, the crucial experiment, is lacking. As an illustration of this, I would refer you to a branch of scientific knowledge which is not included in the special lectures given at this meeting; I allude to the causation of disease by micro-organisms. It is not enough to observe that any given disease is, under certain conditions, communicable from one animal to another, and is therefore contagious; it is not enough to observe that the disease is associated with the presence and multiplication of special forms of micro-organisms in the body tissues; the crucial experiment is still wanting. This consists, first, in the isolation of the observed micro-organisms from all. others, and is attained by causing these, and these only, to develop outside the body in appropriate media. It consists, in the second place, in the introduction of such isolated micro-organisms into the tissues of a healthy susceptible animal. If, as the result of this introduction, the disease is developed, then the crucial experiment is decisive, the judgement is convinced, and the causative sequence is so far determined. It was the genius of Koch which, realizing the cogency of such experimental procedure in the case of anthrax (wool-sorter's disease), first proved that this disease was definitely due to the introduction 
into the body of a specific microbe (bacillus anthracis), and thus laid the foundations of the now extensive science of bacteriology in its application to the science of medicine.

The convincing nature of every such crucial experiment is dependent upon its freedom from sources of error, suspected or imperfectly known at the time; in the carrying out of such experiments the scientific man labours with a special sense of his intellectual and moral responsibility, and the fear of error thus necessitates that the same experiment should be repeated over and over again. In the example I have selected there are various considerations which make this responsibility obvious to the public at large, but a similar responsibility attaches to every experiment carried out for the furtherance of scientific knowledge, necessitating its frequent repetition under similar and under changed conditions. Nor does the responsible duty of the scientific man end here, for the fear of leading others astray is ever pressing upon him and demands that his description of his work shall be in language stripped of all ambiguity, language which shall set forth the experimental procedure and the results in their naked truth. The character of scientific work, with its expenditure of time, labour, and even wealth upon what the world regards as trivial detail, thus requires from him who employs the scientific method ungrudging service; thus only can he aid in constructing the slowly rising temple of science. 'The philosophy I recommend,' says Robert Boyle, 'is much more difficult, laborious, and expensive than that of Aristotle and the Schools.' In dignified language Boyle shows the world and all his carping critics that his own spirit was one prepared at all hazards to undertake its pursuit. 'I am content,' says he, 'provided experimental knowledge be really promoted to contribute to the meanest way to advance it, and had rather be an under-builder 
and even dig in the quarry for materials towards so useful a structure than not assist in erecting it.'

The extension of the field of science by logical inference from known phenomena.

It rarely, probably never, happens that all the members of any causative sequence become known; there remain gaps in the chain, but the scientific method, with its continual appeal to reason, not infrequently suggests the character of the unknown links. The field of science is thus extended and new methods of observation and experiment are devised in the confident expectation that hidden phenomena of the type inferred will be revealed by their means.

Sometimes, however, it happens that the known phenomena are of so cogent a character that a generalization, including those which are at the time unknown, can be made with logical certainty, a deduction sure to be verified when the appropriate means for further discovery become available.

It is at first sight curious that such convincing scientific demonstrations, with logical certainty as their intellectual guarantee, should arouse more intense if less general opposition than scientific theories or hypótheses.

But it must be remembered that this is, after all, a characteristic of human nature. It is referred to by Glanville in the Vanity of Dogmatising as 'men's backwardness to acknowledge their own ignorance and error though ready to find them in others'. So long as an element of doubt is admissible an opponent will suffer the inference to be drawn without a violent outburst; but when the inference is logically certain and the opponent is forced to admit his error or stultify himself, then he may, it is true, maintain a magnanimous 
silence, but generally he fails to do so; he becomes greatly perturbed, and denies everything, even the most demonstrable facts. There is no greater incentive to unreasonable anger than the conviction that our position has been shown to be erroneous, and that in our inmost souls we are fully conscious of its hopeless character.

Thus it was with many of the critics of the work of the scientific method; a scientific inference was first branded as a dangerous perversion of traditional truth; then it was lampooned as a ridiculous absurdity; then it was admitted to be plausible, but, after all, open to grave doubt. And, finally, should all doubt vanish, what course was left but to indicate politely that the reasoner was himself untrustworthy and the description of the phenomena which he adduced a tissue of falsehoods?

From many striking instances of this sort of hostility I select one in my own province of science, the opposition to the demonstration by the illustrious Harvey of the circulation of the blood. The details of this demonstration were set forth in 1628 in the celebrated disquisition upon the motion of the heart and blood in animals. In this Harvey ' marches victorious from position to position until the whole truth is clearly put before the reader'.' The stages in this demonstration are briefly these: the construction and action of the heart which must receive blood only by the veins and pump it out into the arteries; the direction of the actual blood flow in the arteries ever from those parts nearer the heart towards those parts farther off; the construction of the veins containing valves which permitted the blood to flow in one direction only, that being towards the heart. Since the flow of blood continues without cessation during the whole of life only one conclusion is logically admissible :

' See M. Foster, The History of Physiology'. 
this blood always flowing from the heart into an arterial system, and to the heart from a venous system, must find its way across from the arteries to the veins and thus circulate. Harvey's difficulty lay in the circumstance that as the microscope was not in use, no known path existed by which the blood could be conveyed from the smallest arteries into the smallest veins; there was a gap in the vascular series, but his demonstration made it a logical certainty that a bridge across this gap was in existence. The convincing nature of the argument must, one would have thought, have made the circulation of the blood a logical necessity to every thinking man; yet though Harvey lived to his eightieth year he hardly witnessed his great discovery established before he died; and it has been said that he was the only one of his contemporaries who lived to see it in some repute; no physician adopted it, and when it got into vogue they then disputed whether he was the discoverer.

Even men of great intellectual attainments such as Sir William Temple refused to credit the demonstration, ridiculed as absurd the doctrine of the circulation of the blood, and, to avoid the cogency of the argument, denied the demonstrable facts. Temple finally appealed from reason to that last resort of the baffled antagonist, the verdict of common sense, and on this quicksand based his disbelief. 'Sense,' he says, 'can hardly allow it, which must be satisfied as well as reason before mankind will concur ${ }^{1}$.'

The irony of Temple's appeal is shown by the circumstance that already, before he made it, Malpighi had bridged the gap by means of the microscope and actually seen the phenomenon which common sense would not allow. In I66I, writing to Borelli, Malpighi described

1 Disraeli's Miscellanies of Literature. 
how he had seen 'a certain great thing' in the lung of the frog; this was the blood flowing through transparent capillaries from the small arteries into the small veins.

In reflecting how it comes to pass that men of the intellectual capacity of Sir William Temple should thus run their heads against the brick wall of logical certainty a remark of Tyndall's is suggestive. He said with reference to the somewhat general opposition of clergymen to science, "the leaning towards belief in scientific truth is probably as strong in them as in other men, only the resistance to the bent is stronger; they do not lack the positive element, the love of truth, but the negative element, the fear of error, predominates.' It may have been this fear of error which made Temple appeal to common sense as against scientific reasoning; at any rate such an appeal is a familiar form of shirking personal intellectual responsibility.

It is worth while considering how it comes about that common sense can be appealed to in order to oppose the conclusions reached by the scientific method. By common sense is meant a general consensus of opinion, based upon either the current belief of the majority of mankind or upon apparently uniform sensory experience. When such current beliefs are concerned with the causation of natural phenomena it is notorious that they are accepted on most inadequate evidence, a few striking coincidences being considered as sufficient for a generalization. Moreover, it can be shown that sensory experience is often grossly misleading, and thus even its uniformity does not warrant its being the foundation for general statements as to natural phenomena.

In a very remarkable work published in $\mathrm{I} 66 \mathrm{I}$, Joseph Glanville discusses with great mental acumen the sources of the intellectual errors which mankind is subject to; 
these he regarded as the direct work of the devil and as evidence of his presence in our midst. The treatise is entitled The Vanity of Dogmatising or Confidence in Opinions, manifested in a discourse of the shortness and uncertainty of our knowledge and its causes. It sets forth the various defects in man's body and mind, which were, in his opinion, the necessary consequence of Adam's sin and the Fall. All mankind after the expulsion from Eden thus bears the mark of the serpent, and this is particularly shown by predisposition to error. Among the sources of error upon which he lays stress are, first, the circumstance that our mental powers are limited in as much as we can perceive nothing but by proportion to our senses, and, secondly, the misleading and erroneous character of the knowledge we thus obtain. By a number of apt illustrations he displays 'the imposture and fallacy of our senses which impose not only on common Heads, who scarce at all live to the higher principle, but even more refined Mercuries who, having the advantage of an improved reason to disabuse them, are yet frequently captivated by these deceiving Prepossessions.' Glanville instances as a 'sensitive deception' the quiescence of the earth. 'Sense,' he says, 'is the great inducement to its belief; its testimony deserves no credit in this case, though it do move, sense would present it as immovable.' This and other 'deceiving prepossessions' are 'in propriety of speech not due to the senses themselves being deceived, the senses only administer an occasion of deceit to the understanding.'

Modern physiology has revealed the complex source of erroneous sensory perceptions, which have their origin, not only in the delusive character of the mental mould causing an error of sensory judgement, but also in the imperfections of the sensory recipient organs; both the 
physiological response of the nervous system and the psychological setting of consciousness thus tend to deceive us. It is the remarkable characteristic of such sensory deceptions that they still persist even when we realize from other evidence that they cannot be in accord with the fact. This is particularly the case in regard to errors of visual perception. The sun or moon appears to be very much larger when near the horizon than when floating in the zenith, yet when the angular distance, subtended by its diameter, is measured under the two conditions there is no difference. The enlargement is not a real one, nor even due to such optical effects of the atmosphere as produce the familiar colour changes; it is an error of judgement arising from the fact that when near the horizon earthly objects of known size and at known distances are viewed at the same time, and a comparison is permitted which the zenith position does not allow. It is easy to prove this, for if the rising moon be viewed through a small hole in a card, the aperture being so fine that all earthly objects are excluded, then it appears to have its zenith size. There are many examples of visual deceptions in which the primary error is in the sense organ. Thus two lines of equal length can be made to appear unequal by putting arrow-heads on each end of one and arrow-tails at each end of the other; an arch can be distorted by placing over one of its segments an upright bar; two parallel lines can be bent in or out by placing an oblique series of short parallel stripes across each line, the series inclining in the opposite sense athwart each line.

Nor are such deceptions limited to visual impressions; the feeling of contact due to pressure is increased if the pressing object is cooled, decreased if it is warmed, so that if two pennies, one hot, the other cold, are placed 
on the forehead they appear to be of different weight, although we know them to be equal. These examples, out of many which might be cited, will be sufficient to show the fallibility of the senses. It is clear that we can only trust the evidence of sensation with great reserve, and it is, therefore, not surprising that the conclusions reached by the scientific method with its appeal to reason should, in many cases, be opposed to those derived from unsifted sensory impressions. There is in consequence a real basis for the conflict between scientific deductions and common-sense judgements, since the latter are largely the general opinion based on sensory perceptions, and accepted without that correction which mature reflection affords. As Glanville says, 'opinions are the rattles of immature intellects, but the advanced reasons have outgrown them.'

\section{Imagination as an aspect of the scientific method.}

I have reserved to the last this aspect of my subject; it is in many respects, and particularly in this age, the most interesting of all. The scientific method has been adversely criticized because, not content with observations and experiments on natural phenomena and with deducing from these a scheme of causative sequence, based on the authority of reason, it leaps beyond the domain of pure reason and enters that of the imagination. To those who have never read it let me commend such lectures as that 'On the scientific use of the imagination', delivered by Tyndall before the British Association in $1870^{1}$. I will quote a few scattered passages from this and other essays to give you a foretaste of their quality.

'Philosophers may be right in affirming that we cannot

1 Tyndall, Fragments of Science, vol. ii. 
transcend experience, but we can, at all events, carry it a long way from its origin.' 'We are gifted with the power of imagination, and by this power we can lighten the darkness which surrounds the world of the senses. Bounded and conditioned by co-operant reason imagination becomes the mightiest instrument of the physical discoverer.'

'There is in the human intellect a power of expansion, I might almost call it a power of creation, which is brought into play by simple brooding over facts,' 'the spirit brooding over chaos.'

Tyndall contrasts the unimaginative with the imaginative scientific investigator.

'To two men,' he says, 'one educated in the school of the senses, who has mainly occupied himself with observation, and the other educated in the school of imagination as well, a bit of matter will present itself differently. The one descends to it from his molar heights, the other climbs to it from his molecular lowlands. ... As regards the appreciation of those most minute forms of life revealed by the microscope, to one of these men they naturally appear conterminous with the ultimate particles of matter, and he readily figures the molecules from which they spring; with him there is but a step from the atom to the organism. The other discerns numberless organic gradations between both; compared with his atoms the smallest vibrios and bacteria of the microscopic field are as behemoth and leviathan.'

'It is plain that beyond the present outposts of microscopic inquiry lies an immense field for the exercise of the imagination. It is only, however, the privileged spirits who know how to use their liberty without abusing it and who are able to surround imagination by the firm 
frontiers of reason that are likely to work with any profit here.'

In this forcible and eloquent way Tyndall introduces his subject; but let me impress upon you that although the imaginative aspect becomes conspicuous in the scientific conceptions of specially gifted men, it is in reality an essential feature of all scientific research. Its scope may be limited, as in ascertaining the more immediate relationships of some restricted group of natural phenomena, but since this involves passing from the known to the unknown, even though the passage is a step, the imaginative gift which enables the passage to be made is essential.

When, however, the passage from the known to the unknown is of a character which far transcends present or even possible experience, then the daring nature of the flight, impressing in its turn the imagination of mankind, exacts homage for the creative power thus revealed.

I select, in order to illustrate this aspect of the scientific method, one or two of those scientific conceptions (law, hypothesis and theory) which have changed the thought of mankind.

Newton's enunciation of the law of gravitation is so familiar that I might merely mention it, but I desire to do more than this, because I do not find myself in complete accord with the view already put before you by my colleague, Professor Case, who has selected this as a typical example of logical deduction. For my own part I regard Newton's statement that every particle of matter in the universe exercises an attractive force on every other particle which varies inversely as the square of the distance between them, as an induction, the conception $D$ of a creative mind gifted with imagination. Professor 
Case himself stated that Newton passed from terrestial to celestial mechanics. In the language of Tyndall, this 'passage from a falling apple to a falling moon' was a astupendous leap of the imagination, for his enunciated law applies in conception to the universe, thus extending into boundless space and persisting through endless time.

The hypothesis of the luminiferous ether is, without doubt, a conception of the imagination. It was first suggested by Hooke in 1682, but was set forth in its present form by the Dutch physicist, Huyghens, in 1690 . It postulates the existence of an all-pervading medium, having properties unlike those of gross matter; boundless in extent, it has no weight, and is in this sense immaterial, hence it offers no resistance to the passage through it of material bodies. It is further conceived as the seat of disturbances, which are propagated as etherial undulations or waves in all directions with enormous velocities and under varied complexities of form. Although absolutely a creature of the imagination, the hypothesis has acquired scientific validity through the circumstance that it renders the complex phenomena of light, heat and electricity susceptible of causative explanation, whilst a further guarantee of its possessing a rational basis is afforded by the innumerable instances of its employment having resulted in verified predictions.

The theory of the conservation of energy was first propounded by Mayer in 1842, and established on its present wide basis by Joule. It is a conception fraught with results of immense practical utility, whilst it has produced momentous changes in human thought. 'Broadly enunciated, the principle of the conservation of energy asserts that the quantity of what we term force in the universe is as unalterable as the quantity of matter; that it is alike impossible to create force or to 
annihilate it.' In order to realize the extent of the imaginative swoop which the intellect of Mayer made in formulating this conception, we have only to follow the history of its birth, as set forth by Tyndall ${ }^{1}$. In the summer of 1840 Mayer, practising medicine in Java, was struck with the brighter red colour of the venous blood of his patients. Reasoning on this he conceived it possible that the brighter colour was due to less bodily oxidation being necessary to keep up the body temperature in hot climates. This drew his attention to animal heat, thence to heat production in relation to mechanical work, and, finally, to all forms of force. From extensive researches along these lines he formulated the theory that throughout the universe, both in the inorganic and the organic world, there are forces which are convertible but are not destructible.

The theory has been extended since his day. Energy, formerly termed force, is now conceived as modes of (c) motion; differences in the mode affect our sense organs so that we distinguish one as heat, others as light, electricity, chemical action, the movement of masses, gravity, \&c. We further postulate the existence of modes which are unable to affect our sense organs; these we can appreciate when transformed into those modes which will affect us. This motion is not in itself perceptible; it is subtle, occurring, for instance, in such a medium as the hypothetical ether. Any given effect which its existence produces upon our senses may cease as a stone ceases to fall when it reaches the ground, or a lamp ceases to burn when the oil is all consumed, but in accordance with the conception of the conservation of energy there is no real cessation of energy motion, there is only an alteration in its mode; thus the sum total remains for ever the same,

${ }^{1}$ Tyndall, Fragments of Science, vol. i, 'The Copley Medalist of $187 \mathrm{r}$.' 
one mode changing to another without any energy ceasing or being lost in the transformation.

Such an imaginative flight is far beyond all sense experience. To the thought of a scientific man the universe, with all its suns and worlds, is throughout one seething welter of modes of motion, playing in space, playing in the ether, playing in all existing matter, playing in all living things, playing, therefore, in ourselves. Now locked together in more intimate embrace, potential energy, now unlocked and streaming as kinetic energy through space, continually alternating between these two settings, this eternal motion never ceases, is never dissipated, and is never recreated; it simply exists. The conception thrills the imagination like a poem. It is probably for this reason that it is always associated in my mind with the beautiful sonnet which Wordsworth composed as he walked the beach at Calais :-

It is a beauteous evening, calm and free,

The holy time is quiet as a Nun

Breathless with adoration; the broad sun

Is sinking down in its tranquillity;

The gentleness of heaven broods o'er the sea:

Listen! the mighty Being is awake

And doth with his eternal motion make

A sound like thunder everlastingly.

The use of the imagination in science has evoked criticism, not so much in the mind of the general public as in that of scientific men themselves. The passage, in a majestic swoop of almost boundless extent, from the known to the unknown is felt to be travelling so far outside present, and even possible, experience as to run the risk of losing that sure foothold on the solid ground of phenomena from which the daring leap was made; it 
seems to many an entry into the misty maze of metaphysics. Until, by the help of the new conception, hitherto unperceived causative sequences are brought to light, this criticism is probably justified.

It is quite otherwise with the generality of mankind, for, provided such creations of the imagination are sufficiently impressive and sufficiently understood, they secure the acceptance of scientific generalizations even by those who do not pretend to a knowledge of scientific methods. The greater the immensity of the conception the more potent its sway over the thought of mankind. The sun moves visibly around us, and night follows day, yet all educated people, however hostile to science, accept the belief that this is because the earth revolves on its axis once in twenty-four hours. Natural selection and evolution, not recognized at first as involving imaginative conceptions of a most impressive kind, passed through the entire gamut of hostile criticism; they were against nature, subversive of traditional authority, ridiculous, opposed to common sense, and based on erroneous data. Finally, when their imaginative aspects were realized, although still criticized by some scientific men as involving too heavy a call on time and matter, they have been adopted so extensively by the public at large that they have become familiar aspects of all modern thought and expression. The conceptions, which science owes to the genius of Darwin, are used to indicate the predominant feature in the history of races, nations, society, religions, professions, trades, commerce, and manufactures. I have even seen the phrase, 'evolution through the survival of the fittest,' applied to the free-wheel bicycle and the rubber-cored golf ball. This use, I might almost say this misuse, of the terms which express definite scientific conceptions, affords a 
striking illustration of the extent to which emotion rather than reason sways the mass of mankind. Science, being pre-eminently a rational system of thought, does not as a rule influence men's actions or determine their beliefs; but when, though still based on reason, it becomes glorified by the touch of imagination and extends our mental view of the world beyond the limits of possible experience, then mankind acknowledges its authority and utilizes its conceptions. Imagination is the rider on the white horse; there is given unto him a crown, and he rides forth conquering and to conquer.

In conclusion, let me briefly summarize those features of the scientific method to which I have drawn your attention. The scientific method is the means best adapted to secure the end for which the scientific spirit strives, the setting forth of an intelligible causative explanation of natural phenomena. The use of the method demands from him who would employ it aright, that he should regard Nature as an aggregate of phenomena all potentially capable of such causative explanation, that he should deem no natural phenomenon too insignificant or too ignoble for investigation, that he should grudge neither time nor labour in making and repeating observations and experiments, that he should have the fear of error constantly before him, and that he should be unaffected by any consideration as to the immediate practical utility of his work. Free inquiry into Nature conducted along these lines, guided throughout by man's most priceless possession, reason, and illuminated by his gift of imagination, has advanced scientific knowledge in the past, and will surely continue to advance it in the future. Thus, through the scientific method, the scientific worker may attain the crown of labour and realize the joy of achievement. 


\section{III}

\section{PHYSIOLOGY; ITS SCOPE AND METHOD \\ (C. S. Sherrington, D.Sc., LL.D., \&c.)}

F IN Nature one of those rare places at which there seems, owing perhaps to the imperfection of our knowledge, a distinct gap in the long chain of creation, lies as a boundary between living things and things without life. Physiology may be described as the study of the working of living things: In this animate field of Nature the physiologist is a student somewhat as the engineer is a student of inanimate machines. The living machine, like those of the engineer, produces workmoving itself and things-and heat-hence our body's warmth-and electricity, \&c. The living machine wears too as it works, but, more complex than the machines of the engineer, it restores its parts as it works and much of its own labour goes in renewing its own living fabric from suitable dead material which we call food.

The engineer studies his machines that he may drive them to the best advantage, that he may if they break down repair them, that he may make new ones better than the old. The physiologist in reverent study of the living machine knows-no one so well-he cannot create another, let alone construct improvements on the old. Yet among his practical aims one is the 
acquisition of knowledge how best to keep the body from damage as it works, active and healthy.

The world of life falls into two provinces, plant and animal. In both the same principles underlie the running of the machinery. In detail they diverge and in practice physiology studies them to a large extent apart. But one truth emerges from these allied divisions of physiology taken together, and that is the manifold interlocking of the actions of plant and animal in the economy of nature. As the world stands the one kind of life seems impossible without the other. The evolution of each has been a condition of the evolution of the other. Their differences are to a large extent complemental. In a plantless world the animal would die of inanition. The crowning beauty of plant-life, the blossom, is meaningless without the animal to fertilize it.

Our glance at a great science must be brief. Perhaps we may win more of its significance if we confine our attempt to some single fractional problem and broadly examine that. It matters little which of the innumerable inquiries opened by Physiology we take. Reference was made just now to the warmth of the body, and we may consider that, since there is no more salient phenomenon in the physiology of animals.

Our bodily warmth is not constantly in evidence to our consciousness, but the simplest observation at any time suffices to demonstrate it to ourselves. We have simply to touch the forehead with the finger and we recognize that the body is warmer than the air in which it is habitually immersed. Physiology seeks to understand how this warmth of the body comes about, its meaning in the sense of its 'how'.

Heat is evolved in the majority of chemical reactions. Experiment finds evidence that the body is the scene 
of ceaseless chemical action; and in the animal body chemical changes which evolve heat-are exothermic-immensely preponderate in the total chemical activity. Closely bound up with the warmth of the body is 'respiration'. Now so cardinal are chemical data regarding the living body for the science of Physiology that physiological search actually foreran the course of purely chemical inquiry in regard to the nature of air. It discovered in air the all-important element oxygen a century before that element was otherwise discovered. It did so by investigating respiration. Mayow by his physiological experiments here in Oxford in 1674 showed air-until then accepted as a homogeneous entity, the second of the four natural elements of the ancients-to be a mixture of two aerial substances, one of them (that later known as oxygen) retained by animals in breathing and an essential support to life. Mayow also showed that it is this same aerial component which supports a flame in burning. He showed that both the animal in living and the flame in burning return to the air, in place of the component they remove, a third gaseous substance heavier than that, a substance unfit to support the flame or life, indeed on the contrary nocuous to animal life.

A century later the stupendous work of Lavoisier founded modern chemistry. Then it became clear that at the bottom of the lungs the blood as it streams past takes oxygen from the air. The fish in river and sea also take oxygen, drawing upon the relatively scant quantity of that gas dissolved in such water. But our blood and theirs take more than they can hold in mere solution. Minute globules suspended in the fluid of the blood have power in virtue of an iron-containing pigment to seize the oxygen and hold it chemically. 
This pigment changes from purple to scarlet as this occurs. Oxygen thus continually drawn from the environment is offered through the circulation of the blood to every organ in the body. Each organ takes it thence, and takes the more the greater its activity. The mere colour of the blood reveals whether it holds much oxygen or little. So great is the avidity of the organs for oxygen that they act as chemical reducers. Methylene blue loses its colour under the activity of reducing substances. If an animal into whose circulation methylene blue has been introduced be sacrificed, none of the pigment appears on first examination of the organs to have reached a number of them. Only after exposure to the air do they become blue. The pigment had reached them, but so great was their avidity for oxygen that it existed in them in its colourless reduced condition, until their surface was exposed directly to the air.

What is the meaning of this avidity for oxygen? The tie is close between the body's intake of oxygen and its warmth. The body is built of substances chemically unstable, and it is essential for life that they be so. They break down, and in doing so liberate energy engaged within them until then in so-called potential form. They are complex and they break down variously. They crumble gradatim. Every downward step in their decomposition liberates heat that contributes to the temperature of the body. Manifold substances arise from them in this degradation, and these vary in different organs and probably at different times. But the end-results of the decomposition are practically invariable. The end-products are relatively simple. It was one of these that John Mayow had before him in his heavy irrespirable gas, given out alike by the animal in living and by the flame in burning-carbon 
dioxide. Another is water. The large quantity of oxygen in these end-products suggests the rôle oxygen is playing in the decomposition of the living matter.

The need for oxygen-the oxygen hunger-is acute in ourselves and in all briskly living animals. Ten minutes, even five, without oxygen and certain organs, notably the brain, strike work-entailing in sequence the upset of other parts of all the linked machinery of the living body. Breathing ceases, and slowly there follows coldness, the absolutest sign of death. Oxygen being excluded from its part, the normal chemical decomposition ceases; and the complex material of the body will fall a prey to forms of decomposition other than those associated with life, though the end-products may be ultimately the same.

What, then, is the nature of this material that, give it oxygen, so composes and decomposes as to manifest the phenomena we sum as 'life'? Chemical physiology reveals a thing unexpected to many, though a fulfilment of much ancient figurative expression. Last century the chemist performed a mighty analysis, testing the nature of the materials of all the million-sided universe perceptible to man. He learned that despite all its diversity it is yet composed from relatively few essential substances, therefore called elemental. This analysis included living matter. It might have been thought that the material basis of life would contain elements peculiar to itself and different from those of the inanimate world. It is not so. Some few of the elements most widely spread in air and sea and rock make up the tale of those which everywhere compose the machinery of life in all its forms. The secret of the chemical difference between life and death lies therefore less in the nature of its elemental ingredients than 
in the manner of the compounding of them. Many illustrations arise. Carbon compounds preponderate not only in plants and animals but in many rocks and soils. Those of the latter dissolve and from their solvents can be obtained as crystals. Crystalline form is some guarantee of chemical purity, and helps the chemist in separating compounds. Now though the body consists of water to the extent of more than half its weight, its carbon-compounds are for the most part not dissolved but exist in a kind of particulate suspension. Unlike those of the rock, they will not crystallize out; and they contain not definite but variable proportions of water. They do not carry electrical charges in the same way, they do not exert the same pressures or exhibit the same tensions. They offer much greater difficulty to present methods of chemical research. They are in a state which is termed colloidal. Not that the living body is devoid of those simpler chemical compounds which are characteristic of the inanimate world. The living body contains colloids mixed with dilute solutions of crystalloids. And this commingling renders the matter still more intricate.

How largely the working of the body is a chemical problem is signalized by the vast effect upon it of minute quantities of certain substances acting chemically within it. A few drops of cobra-venom or of a solution of diphtheria poison, introduced through a hollow fang or a hollow needle, and the whole machinery perishes in a few hours or days. Such an effect emphasizes the delicacy of adjustment of the chemistry of life. We can conceive that the body, chemical machine as it is, may sometimes poison itself in its own working. Fatigue from muscular exertion seems such a case: poisons are produced from the superactive muscles more quickly 
than can be neutralized in the chemical laboratory of the body.

The heat of the body is traceable to chemical decomposition of its substance. The material whence the energy has been thus far extracted is no longer useful to the body. It is got rid of, for instance, in each breath we exhale. Yet our weight keeps up from week end to week end. While that is maintained and the body's average composition kept unaltered, every manifestation of activity by the body is traceable to the energy of its food. The body is thus a fuel-fed machine. The energy we dispense each day, not only as bodily warmth but in all our various activities, walking, talking, climbing omnibuses and taking cabs, comes in as the chemical energy of food. And the principle which is termed that of the 'equivalence of energy' allows us a precise quantitative idea of the value of food.

We can measure the energy that the food taken by the body sets free when burnt in the presence of oxygen. A representative sample of the total diet is placed in a steel shell which contains compressed oxygen. This shell is immersed in water contained in a vessel so jacketed as to avoid loss or gain of heat from the external air. When the temperature of the whole is constant the sample of food is fired electrically. In being totally burnt in the oxygen, it produces a certain amount of heat. This is caught by the surrounding water. The temperature of the water is taken before and after the combustion. The quantity of water is measured. The amount of heat produced is thus known from the amount communicated to the water. A measure is thus obtained of the quantity of energy available in the food. From this we subtract the energy of a residuum of the food not fully oxidized in its passage 
through the body; this also is estimated by sample in the calorimeter.

This subtraction gives the total energy an animal is extracting from its food. From the law proved by Lavoisier for the inanimate world, namely that in it matter is neither added to nor consumed, life offers no departure. The doctrine which states that energy, though it pass from form to form, can, like matter, neither be added to or consumed in any of its permutations, was likewise drawn in the first instance from study of the inanimate world. Now there is a great deficit of energy obvious when the energy of the waste matter leaving the body is compared with that of the food supplied. If the conservation of energy holds in vital reactions, this deficit will be exactly accounted for and balanced by energy expended by the body to which the food is furnished. The energy expended by the body can be measured. A calorimeter is used, more complex than the one for food values. In such a calorimeter a man may live days at a time and divide his energy between novels and a dummy bicycle. Investigation shows that the quantity of energy coming out of the body exactly equals the deficit of the energy of the material that passes through it. The conservation of energy obtains therefore in our own frame as in the tide, the waterfall, the furnace. It is a law in the living world as well as the inanimate. This it is which encourages the physiologist to feel that in studying the living machine he has assured at least the source of energy whence all its powers are drawn.

But the engineer regarding his machinery knows also 'how' those powers are drawn. Of this in the body we have far less knowledge. Food is not fuel in the ordinary sense. The fuel of the gas-engine becomes no part of the 
fabric of the machine, and is not built into it, repairing wear and tear. But food entering the body seems to be in large measure vivified before its energy is taken. It is built up into the living fabric of the body. In that sense the creation of living matter from dead-a literal though not the technical heterogenesis-is going on today, and in each one of us.

Seventy years ago the microscope made its greatest discovery. The old Greek simile of our school classic likened man's body to the body politic, the State, a corporate whole composed of individual members. Biology gives this a literal truth. The microscope reveals that plants and animals are literally commonwealths of individually living units, each unit a mere speck in size. Collectively in each one of us they outnumber the whole earth's human population. Thus the corporeal house of life is built of living stones. In that house each stone is a self-centred microcosm, individually born, breathing for itself, feeding itself, consuming its own substance in its living, renewing its substance to meet that consumption, harmonizing with its own inner life some special function for the benefit of the whole, and destined ultimately for an individual death. Day-long, nightlong, in this commonwealth that constitutes each one of us, there goes forward as in the body politic the subservience of many individual purposes to one, the sacrifice of individual lives for the advantage of the many, and the birth of new units which replace the dead. And in all this we ourselves do but resemble each plant and animal we see. And each of these living commonwealths began its individual existence as a single unit, whence arose the myriads that compose its adult being. Division of labour went on and with it differentiation of structure. A plan informed the mass that otherwise 
were a mere congeries of cells. There come thus to co-exist the lime-hardened tissues of our bones, the contractile cells of our muscles, the conductive cells of our nerves, and so forth.

And more than that, the living laboratory of the cell itself manufactures even the medium in which the cells are immersed, not only the fibrous and calcareous framework, but the very saps and juices of the body. We are learning from pathology that every species of animal produces an internal medium, un milieu interne, specific to itself. The difference between the child that, recovering from some disease, has become immune to that disease, and the child that, not having suffered, is not immune, seems perhaps chiefly expressed as a change in this 'internal medium' of its body. From this point of view, the processes of the body fall into two categories, those within the cells and those within the body but outside its cells-in its intercellular material.

And these two are connected by a third, namely by processes connected with cell boundaries. These boundaries limit more or less stringently separate fields of chemical operation: by them spheres of influence for ferment action are confined, osmotic pressures are banked up, ions are checked or sifted, electrical charges are accumulated, and so on. For instance, osmotic energy manifests itself when a soluble substance finds itself unequally distributed in a solvent. If in the living cell disintegration of complex substances go on under the influence of stimuli, in proportion as the number of molecules increases and as the limiting layer of the cytoplasm obstructs their passage osmotic pressure in the cell will rise. Water enters the cells. In proportion as the surface of separation is permeable molecules of chemical products of disintegration diffuse from the cell. 
It is physiological variations of osmotic pressure which determine, on the one hand, currents of water across the cells, and therefore across the tissues of the body, and, on the other, the diffusion of substances suitable for drafting off into definite channels of the body. It is noteworthy that the relation between osmotic energy and the products which the cells form takes no note as it were of the chemical nature of the products, but only of the number of their molecules present per unit of solvent. It has therefore no selective action in that sense. Y Yet it does act selectively in a certain sense, because the lower the stage to which a product belongs in the disintegration of a large complex molecule pari passu the greater the number of molecules of it which will arise from the parent compound. Therefore diffusion by osmotic energy will act more in regard to final products of protoplasmic disintegration than to earlier.

One consequence of the cell-theory is therefore a recognition of the construction of the body as including vast numbers of surfaces of separation, dividing minute chemical and physical fields one from another. Membranes and surfaces deserve special consideration therefore in physiology. In the limiting layer separating two media forces act which depend on mutual relations between the two media in contact. It is the internal energy of such a limiting layer which is usually called the energy of surface. Energy of surface is therefore a form of potential energy. It cannot therefore derive directly from chemical energy, which is also a form of potential energy. A free form of energy must intervene; perhaps heat; more probably electrical energy. Colloid solutions are not homogeneous systems, but suspensions, of ultra-microscopic fineness. That is to say, they are diphasic systems offering vast surface; the limiting surface between the two, the 
particles suspended and the fluid in which they are suspended, may allow the existence of a double electric layer. Such a system can hardly be considered a stable one. It has a tendency to pass into one offering the least possible surface. Why is it then that it does not deposit its suspension in flocks, as when we precipitate a proteid. It may be electrical difference which counteracts this. Surface tension would reach its maximal effect when the difference of potential in the double electric layer, fell lowest.

Solutions of salts usually precipitate colloids; one method of preparing these latter from their suspensions -often called solutions-is by salting them out. Various physiologists have suggested that in the limiting stratum between a living cell and the lymph which bathes it there exists a double electric layer. In the case of a contractile cell-an amoeboid blood-cell or a muscle-fibre, the double electric layer may be the condition which counteracts the tendency for the surface to reduce itself to the smallest possible. The shape of so-called repose of such a cell would be the expressionthe morphological expression-of an equilibrium between the difference of electric potential and surface tension. If a stimulus applied to the cell or muscle consisted-as many artificial stimuli do-in an electrical charge, if that charge lessened the difference of electric potential, the surface tension would act, and the contractile cell would, its volume remaining the same, approximate to that form with the smallest surface, the spherical form. That is, the muscle would contract. And here comes a difference between precipitation of a non-living colloid and the case of the contraction of a living cell. In the nonliving colloid there ensues no subsequent spontaneous resolution-the difference of electric potential is not re- 
generated. But in the living muscle-fibre its chemical action, its metabolism, furnishes energy by which the potential can be re-established to its primitive valuenamely, there is a return to the less stable condition which we in the case of the muscle term its resting condition. To us here the interest of such statements as these is the broad one that, partially speculative though they may be, they serve to illustrate the essentially chemical and physical character of the considerations upon which physiology is based, and the enormous and still almost virgin wealth of opportunity which the cell-theory reveals in the body's structure as a field for the play of chemical and physical reactions of a certain kind.

The cell-theory impresses on us that the chemistry of each organ, let alone of the body as a whole, must be a chemistry resultant from ten myriad tiny foci, each an individual seat of oxygenation, reduction, polymerization, hydrolysis, katalysis, and what not. And returning to the warmth of the body, we see that in it the student has to deal not with one seat of heat production, but with a countless number of microscopic furnaces, with conditions and rate of action very different in some from that which they are in others. The matter is therefore complex to unravel.

Yet certain points are clear. Since the body is warmer than its surroundings, its heat drains from it. The greater the difference in temperature between it and its surroundings the quicker will be the drain. That difference is measurable by thermometry. Thermometry shows that the temperature of the outer surface of the body differs from part to part. That of the face may differ considerably from the hand. But at a small depth below the surface the temperature is practically alike throughout 
the body. The heat produced in the organs is not only conducted through the body but conveyed by currents of the everywhere circulating blood. Just as the heating of a building by hot-water pipes is partly a mechanical problem, so in the warming of the body there is a mechanical factor, namely the circulation of the blood.

The uses of the body which physiology earliest glimpsed were those of mechanical design. The study of bodily form, being relatively simple, was early prosecuted. It was easy by dissection of the dead and examination of the shapes of bones and muscles to infer in outline what would be the mechanical adjustments of those parts in life. $U_{p}$ to the sixteenth century physiology followed merely that crude method, chemistry and physics hardly as yet existing. The form of the organs of the body is significant chiefly where they are machinery for the application of mechanical powers. But the greater part of the corporeal machinery is not mechanical but has its purpose in processes, chemical, thermal, electrical, \&c. Even with regard to muscle the study of its gross form can say nothing on the fundamental question, ' How is its force developed ?' The form of the liver or the pancreas tells us nothing of the function of those great chemical organs; their form fits into the space-conditions of the abdomen. Study of the gross form of the brain has never shed light on the functions of that organ, nor is it likely that it ever will.

But in the middle of the seventeenth century there came to physiology one discovery of a mechanical kind of importance. Besides the ordinary muscles such as move the bones there is one muscle which is hollow. Its cavity opens into the tubes of the arteries and receives opening into itself the tubes of the great veins. This muscle is the heart. Harvey by watching it at work and by experiments 
to test the direction of the-blood-flow along the vessels, and by mechanical inferences from the form of the heart and vessels, learned that the blood circulates throughout the body, passing and repassing through the heart ; and that the heart is a muscular pump which maintains the circulation of the blood.

To realize how the circulation equalizes the temperature through the body we have to remember that in certain organs the blood tends to be warmed whereas in others it is cooled. The blood that streams close under the surface of the skin-and lends that its rosy tint - is cooled in doing so. This on returning to the heart and large blood vessels is mixed with warmer blood from the rest of the body. Thus mixed it is redispersed again from the heart to all parts both of the interior and the skin. The circulation therefore diffuses the warmth equably through the body.

The thermometer shows that our surface temperature not only differs from part to part, but that it varies from time to time; for instance, it rises on our entering a warm room. But inside the body the temperature is found hardly to vary at all. We may travel from the pole to the equator without its varying. And in this we resemble all warm-blooded animals, better called animals of constant temperature.

How is our body-temperature thus kept constant? The temperature of the blood as a whole will depend on how large a fraction of it is cooled by streaming through the skin. The regulation of that quantity is a main means of regulating the deep temperature. It is common experience that we flush after entering a warm room. That is, the blood-vessels of the skin dilate. A larger portion of the total blood streams through the cool skin-zone of the body; the escape of heat from 
the body is more free; this counteracts the tendency for the internal temperature to rise in the warm surrounding. The skin becomes warmer but the internal temperature remains unaltered, or often is a little lowered from over compensation.

Moreover, when we are exposed to warmth the skin becomes suffused with moisture. In the little valleys crossing the ridges sculptured on the skin lie the mouths of tiny glands. Their deep ends lie coiled among the tiny blood-vessels in the deep layer of the skin. These glands constantly secrete water which they draw from the blood. Usually they secrete this so slowly that it evaporates as quickly as it is formed. The evaporation contributes to cool the surface of the skin. Whether water exists in the solid, liquid, or gaseous state of course depends only on the amount of heat in it. The water, as it evaporates from the skin, abstracts heat from it, large in quantity since the specific heat of water is so high. The skin becomes moist in a hot room because its glands become more active, so active that the supply of water is greater than the evaporation. But the evaporation is increased and increases the efficiency of the skin as a cooling zone for the circulating blood.

How is this mechanism worked? What is it causes the vessels to dilate, the glands to secrete more, when warm air plays upon the skin? Does the heat act directly on the vessels and the glands? It is found by experiment that certain nerves control the vessels and glands and cause them to dilate and secrete. These nerves run from nerve-centres in the depth of the body. These centres are too remote from the surface to be acted on by the warmth of the air directly. But the skin has also many nerves which run from it to the nerve-centres. If a lighted match is held near my skin 
and I have a sensation of warmth from it, that is good proof that a nerve-a so-called sensory nerve-runs from the skin there to my nerve-centres and that these are connected with my brain.

A region of skin, the nerve-fibres of which have been severed, does not flush with blood properly when exposed to warmth, nor does it become moist with perspiration. Therefore the course of events in the regulative mechanism is that the heat playing on the skin stimulates nerves that run to the nerve-centres, and that these in turn stimulate nerves which run thence to the bloodvessels and the glands. In fact the mechanism includes nervous reflex action.

Thus in our problem of the warmth of the body nerves are involved. I have spoken rather glibly about nerves, but our knowledge of them is meagre, especially as to how they work. Chemistry is here at a disadvantage, for its difficulties are enhanced by the minuteness of the quantity of material disbursed by nerves; the turn-over of material in them is relatively small.

The smallness of the turn-over of energy and material in the nerves depends largely on the fact that, spread like a delicate network through the body and furnishing as it were telephonic communication between the various organs and regions, their reactions act like releasing forces, themselves small though controlling machinery in which large quantities of force may run. A telephonic message expends little energy, but it may set a train in motion or stop the marching of an army corps. Suppose we knew nothing of the mode of action of telephones and knew concerning a telephonic system only its places of departure and its end-results at various stations. That would represent the position of physiology in regard to most of its observations on the nervous system 
were it not for help from physics. No visible or chemical or thermal sign measurable or even perceptible is given by the functional activity of nerve. The only change known in it is electrical. This in many of its forms is brief and extremely small. The instruments for its study are delicate and require a highly developed technique. To the study of the speed of travel, the period of duration, the intensity, the direction, and so on, of this electrical sign we owe most of what is known physiologically of the working of the nervous system. It is one of the distinctions of the famous physiological laboratory here in Oxford that it has contributed more than any other to the knowledge of this electrical sign of nerve-action. In doing. so it has laid under contribution phenomena from the conductivity of frog's nerve to, on the one hand, the discharging shocks of electric fishes, and, on the other, the nervous discharges of the convulsions of epilepsy. It has found significant parallel between nerve-action and the inter-connexions between distant organs in plants; though plants are beings devoid of nervous system in the ordinary sense.

It is of the highest interest to know what is the relation of this electrical sign to the real function of nerves. Is the real functional activity of nerve a process completely hidden from us except for a by-product, an accessory phenomenon, namely this electrical disturbance which happens to be accessible to us in virtue of superior delicacy in respect of our electrical methods of investigation as compared with other methods applicable in this case? Or is this electrical disturbance the real cardinal action which constitutes the main function which nerve has to perform? It seems-and many have thought it - that it may well be the latter, and that the comparative poverty of chemical findings in regard 
to nervous action may mean that the nervous activity is not immediately based on purely chemical change. In the concentration cell we have an instance of electrical energy which, though arising from chemical solutions, arises from them not from chemical reaction but in virtue of changes comparable with the simple expansion of a gas. If electrical disturbance is the cardinal function of nerve, we are not so little advanced in essential knowledge of the activity of the nervous system as would otherwise appear.

And the factor of nerves in the mechanism of our temperature regulation brings naturally the question; Are our sensations of temperature part of this mechanism?

Now since the temperature of all but the very surface of the body does not change whether we are exposed to heat or cold, it is obvious that when we feel hot or cold the sensation arises not from the depth but from the surface layer of the body. Again, though a man's internal temperature may not be affected one degree in travelling from the arctic region to the equator, yet it will alter much even while he sits at home if he 'catch cold'-he then becomes 'feverish'. At the outset of fever he feels 'chilly', although his internal temperature is higher than usual. A spasm of the blood-vessels of the skin makes the skin cold as it would be in a cold draught. It seems clear that our sensations of cold and warmth arise from nerves distributed to the skin and not to the deep organs.

But it would be wrong to suppose that it is these sensations which regulate the temperature of the body. That regulation, accurate and smoothly-working as it is, is not a volitional regulation. It is automatic. We can assist it, but we cannot govern it further. True, we are disinclined to take active exercise in extreme heat; and, 
conversely, are on a cold day impelled to move about. The mouse and sparrow, their surface whence bodily heat escapes being so large relatively to the small bulk meted to their furnace, exhibit in cool surroundings greater tendency to move about than do large animals such as the ox and vulture. And these movements have the appearance of a conscious motive. Yet the part of the brain employed in the automatic regulation of body temperature is not that part to whose activity the sensations and processes we can introspect are adjunct.

And this automatic unconscious work of regulation is typical of the greater part of the work of a large portion of our nervous system - a portion constantly active in processes quite imperceptible to our minds, imperceptible even if we try to direct the attention of our mind toward them.

But though this regulation of the body temperature is automatic, and occurs in us without our consciously guiding it, it nevertheless is obviously calculated to benefit and to protect the vital machinery from harm. The same is true of all physiological reactions. They are adjustments to environmental vicissitudes, and are adapted to conserve the well-being of the organism. Darwin showed how the wide-spread existence of this property of utility in bodily reactions can be explained as adaptations under what he termed 'natural selection'. To know somewhat of the 'purpose'-if I may be allowed that term in this connexion-which the reaction serves is of much help in studying a physiological reaction. But physiologists, though aware of the potency of such assistance, are rightly cautious in resorting to it, since the 'purpose' of the reaction is often, in our present meagreness of knowledge, very difficult to decipher, and mistakes may be made regarding it. 
A point, and this must be our final one, is however raised by the sensations connected with the body's temperature. Phenomena inseparable from changes in the temperature of the skin, we have met them as it were naturally and perforce in the course of our physiological inquiry. But what of our comparison between the physiologist and the engineer as both students of machines? Are sensations part of the output of the living corporeal machinery? Does, then, sensation come in under the conservation and equivalence of energy? That is a form assumed to-day by the perennial question of the relation of mind to matter. It is debatable whether such a question belongs to Physiology. I mention it merely to note that the very obviousness with which it intrudes itself in the pursuit of our physiological argument illustrates how boundaries of natural sciences exist, like those of natural species and descriptive provinces of all kinds, largely as human conventional distinctions, no more than and just so much worthy of observance as they may serve utility. 'Sensation' is a subject matter of Psychology, but it seems useful at this present that physiology and psychology should overlap to some extent in their investigations.

What then from our brief sketch may we conclude regarding Physiology, its scope and method? I think

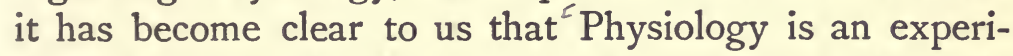
mental science, for by means of experiment she puts her conceptions to the touchstone of fact; and that Physiology is a branch of energetics, though morphology in many cases shapes her field. She traces the current of energy constantly flowing through that part of Nature which we term alive. She finds the individual life a sort of eddy or node in a great stream of energy, an eddy checking an infinitesimal fragment of that stream, for 


\section{PHYSIOLOGY; ITS SCOPE AND METHOD}

a space of time which is almost less than a moment compared with the cosmic period through which that stream in its relentless descent towards dissipation must run ere it reach the zero of the equilibrium that shall be final. In that eddy the energy is diverted to a course which taken in its totality has no terrestrial parallel. And each such eddy, though of brief existence, throws off from itself-and apparently this is one of its main 'purposes' - the beginnings of other eddies like to, but not identical with, itself. We can see that Physiology must, in order to study the properties of these eddies, be an eclectic study, borrowing her knowledge and her methods from many cognate studies. 'It can be said of her that she has no methods of her own, or that all methods are hers: either expression is true. What is hers and hers alone is the scope of her problem, namely, the riddle of how the living organism lives. $]$ 


\section{IV}

\section{INHERITANCE IN ANIMALS AND PLANTS}

(Professor W. F, R. Weldon)

I HAVE been asked to tell you something about the study of Inheritance in Animals and in Plants, about the difficulties students of Inheritance have to contend with, and the special ways in which they try to meet these difficulties. I shall try to tell you what I can about Inheritance presently; but before I try, I must ask you to think for a while about one or two preliminary matters.

'It is the first business of a scientific man to describe some portion of human experience as exactly as possible. It does not matter in the least what kind of experience he chooses to collect; his first business is to describe it. Those differences between the methods used in the different sciences which depend merely on the different kinds of experience with which they deal, and upon the different ways in which these kinds of experience have to be collected,--such differences are merely matters of technical detail, and we need not discuss them to-day. But there is a more important difference between the different kinds of experience which students of science collect, and that is, that some kinds of experience can be described more exactly, and can serve as a basis for more 
exact prediction of future experience, than others. This difference in the degree of accuracy with which different kinds of experience can be described has a really important influence on the methods employed in different sciences; and before I can attempt to show you how the methods employed in the study of organic Inheritance differ from those employed by an astronomer or a physicist, I must ask you to consider for a moment what experimental exactness means, and how nearly it is attainable by any one.

The only way in which I can show you what absolute exactness means, is by reference to a science which does not appeal directly to experience at all-the science of Pure Mathematics. There is a certain geometrical statement, familiar to all of you, to the effect that the three angles of a plane triangle, taken together, are equal to two right-angles. I dare not guess, nowadays, how you convinced yourselves that this statement is true; but I want you to think of the way in which Euclid shows you that you must believe it. He first gives you definitions of straight lines, planes, and right-angles. He does not tell you to get a plane and to draw straight lines and rightangles on it for yourselves, because you cannot draw the things he defines, and if you had drawn them you could not see them. But Euclid says that so long as he is speaking to you, his words will have the meaning given in his definitions; and although these definitions describe nothing within the range of your actual experience, you can follow their meaning sufficiently well to use them as instruments of thought, just as you can think of a mermaid, or a centaur, though you have never seen either. Well, the next thing Euclid does is to tell you a few truths so simple and so obvious that the moment they are uttered you recognize their truth, and agree that they are 


\section{AND PLANTS}

axiomatic $^{1}$. Having done all this, he makes you believe his story about the sum of the three angles of a triangle by showing you that unless you believe it you must either deny one of his axioms, which your instinctive recognition of its truth compels you to believe, or you must refuse to accept one of his definitions. Now, if you can deny the axioms to which Euclid appeals, the laws of your thought are so different from those which govern the thought of normal men and women, that you must make beliefs of your own: neither Euclid nor any normal person can help you. And if you refuse to accept the definitions, you do not invalidate Euclid's statement, you only insist upon talking about something else.

I am not going to discuss the very difficult question how it has come about that the truth of Euclid's axioms is self-evident to all of us, and I shall not discuss the way in which he was led to conceive his definitions of straight lines and the rest. But, however these things may have arisen, the definitions at least have now to be accepted without any conscious appeal to experience, and the process, by which we deduce the properties of a plane triangle from them, involves no appeal to experiment, either for its performance or its subsequent verification., Euclid's statement is a description of a mental process, and it would be just as true as it is, though it would not be quite so interesting to many of us, if there were nothing in our experience remotely like the plane triangles he describes: every time we went through the mental process he tells us to perform, we should still arrive at exactly the same result, and his proposition would still be exactly true.

1 Speaking broadly. I do not, of course, wish to enter here on a detailed discussion of the question whether they are all axiomatic. 
But the word Geometry emphasizes the fact that our experience does include a knowledge of things which are sometimes very nearly indeed like Euclid's triangles. Such experimental triangles play a great part in many scientific investigations, and I cannot find a better way of showing you the difference between attainable experimental exactness and the absolute exactness which results from following accepted definitions to their logical conclusion, than by saying a word or two about our knowledge of these experimental triangles. If we measure the three angles of one such triangle as accurately as we can, and add the results together, we shall find that the sum of these angles is very nearly indeed equal to two rightangles; but it will generally differ by some small amount from the exact sum which we find when we deal mentally with Euclid's triangles. The first case of such a measurement I found, when I looked for an example of this, was one in which the observed sum of the three angles contained $180^{\circ} \mathrm{O}^{\prime} \mathrm{I} 2^{\prime \prime}$, instead of exactly $180^{\circ}$. This is a very close approximation to Euclid's result, differing from it by only one part in 54,000 , but it is not exactly Euclid's result, and the question arises, whether this small difference between one result and the other depends on some difference between the properties of the two kinds of triangle or not. Now the natural thing for an observer to do, after he had found that his sum differed from Euclid's by twelve seconds of arc, would be to measure his triangle again, in order to see if he had made any mistake. If he did this, it is very unlikely that he would find exactly $180^{\circ}$, but it is also very unlikely that he would find exactly $180^{\circ} O^{\prime} \mathrm{I} 2^{\prime \prime}$. If he made a great number of measurements, he would find that some one or more of them gave every possible value within a small range, outside which none of them would fall; and within this small 
range he would be unable to predict the result of any new measurement, however often and however carefully he had measured his triangle before.

If I tried to show you the results of measuring the three angles of a triangle a great many times, I should probably tire you very soon: I shall therefore only ask you to look at measurements of a single angle, so that you may realize the sort of approximation to a constant result which skilled observers can reach when they make repeated measurements of what seems to be a constant angle.

Some of you know the Radcliffe Observatory in the Woodstock Road. In it there is a telescope, with which important observations have been made, and astronomers all over the world are anxious to know the position of this telescope on the earth's surface as exactly as possible, in order to compare their own observations with those made through it. Accordingly, successive observers have determined the latitude and longitude of that telescope with great care. You know what the latitude of that telescope is. It is the angle between two imaginary straight lines, which start from the centre of the Earth, and come to the surface in the meridian of Oxford, one coming out at the Equator, and the other through the telescope; so that determinations of the latitude of that telescope are attempts to measure an angle. In the Nautical Almanac, and in various other publications, the latitude of the Radcliffe telescope is given, together with that of many other instruments; but if you look at these various publications, you will find the value given for the latitude of the Radcliffe telescope is not always the same.

Between 1840 and 1887 the Radcliffe observers published their own estimates of their latitude, with this result :- 
Determinations of the Latitude of the Radcliffe Transit Circle.

Year. Latitude.

I $840-5 \mathrm{I}^{\circ} 45^{\prime} 35^{\prime \prime} \cdot 85$

I84 I — " \# $35 \cdot 97$

$1842-", 35 \cdot 70$

r843-", "35.82

1854-”, $35 \cdot 31$

$186 \mathrm{I}-", \quad, 35 \cdot 88$

I862- ", 35.85

1863- " " $35 \cdot 73$

I864- " " $35 \cdot 50$
Year. Latitude.

$$
\text { I } 865-5 \mathrm{I}^{\circ} 45^{\prime} 35^{\prime \prime} \cdot 28
$$

I880- " " $35 \cdot 17$

I88ז- " " $34 \cdot 95$

r 882 - " " $35 \cdot 24$

I $883-", \quad 35 \cdot 23$

I884-", "35 49

$1885-", \quad 35 \cdot 43$

1886- " " 35.67

1887- " " $35 \cdot 73$

You see that up to a certain point, all the experience of this angle, acquired and recorded by highly trained observers during nearly half a century, is constant. The latitude is always greater than $51^{\circ} 45^{\prime}$, and nearly always greater than $51^{\circ} 45^{\prime} 35^{\prime \prime}$; but when these men try to record observations of less than a second, their successive records do not agree. The difference between the highest and the lowest values of the latitude observed is $\mathrm{I}^{\prime \prime} \cdot \mathrm{O} 2$, which corresponds to a distance of about thirty-four yards measured on the Earth's surface, North and South through the telescope; and the remaining values are scattered between these limits.

Now although thirty-four yards is a very sensible distance, considered in itself, it is a very small fraction of the distance on the Earth's surface from the Radcliffe telescope to the Equator. A line drawn due south from that telescope to the Equator would be about 6,300,000 yards long, so that the extreme range over which uncertainty about the result of any one measurement has been shown to extend is less than one part in 180,000 of the whole magnitude measured. This may be taken as a type of a very excellent experimental approximation to a constant result. We can predict the result which any properly trained person will get, if he measures this angle of latitude again, to within a very small fraction of the 
whole angle: but beyond a certain limit, we cannot do so.

For most purposes, the people who want to know the latitude of the Radcliffe telescope are content to take a value somewhere within these very narrow limits, and to neglect the observed discrepancies. The value chosen for the latitude published in the General Catalogue of Stars (1890) is about fifteen yards North of the lowest observed latitude, about nineteen yards South of the highest. This value was selected by a well-known process, but I want you to realize clearly what we do when we adopt it. We reject a carefully made record of a variable experience, and substitute for it an ideal record of a constant experience which we have not attained. Why do we dare to do this? If you ask an astronomer this question, I suppose he will answer you by saying that he wants to use the position of the Radcliffe telescope in calculations which enable him to predict some future experience, say of the relative positions of stars. But he will not be able to measure that experience accurately, and the differences in his predictions, involved by taking into account all the various observed values of this latitude, would be so small that he could not verify or disprove them by his subsequent measurements, and therefore it is not worth while, as a practical matter, to take them into account. This is a perfectly reasonable statement, and its justification lies in the accuracy with which future experience can be predicted by the process adopted. But if you ask your astronomer whether he believes that the constant value he has chosen to adopt represents a real constancy in the angle of latitude itself, his answer will be more difficult to follow. Some of the discrepancies between the observations he will certainly attribute to the observers ; and if you think 
this implies that they are incompetent, I advise you to measure a few angles so that you can be sure of the minutes; you will then begin to have some idea what measuring an angle to a hundredth of a second means. But while some of the uncertainty about any particular determination of latitude is due to the slight inaccuracy of the instruments, and to the fact that no one can read them quite exactly, yet by carefully comparing the observations of latitude made at different places at the same time, it has been shown that the position of the axis about which the earth rotates, and therefore the position of the equator, does in fact change to some small extent from time to time, so that when we replace the record of variable experience by a constant, we are attributing to the latitude of the Radcliffe telescope a constancy it does not really possess.

Now, all experimental constants whatever are determined in essentially the same way as the latitude of the Radcliffe telescope is determined. Men measure a certain thing, and find that up to a certain point their measurements agree with each other, and their experience is uniform; but beyond that point, their experience is contradictory. Having made this contradictory record with as much care as they can, they substitute for it one constant value of the thing measured. But the question always arises, how far this proceeding is justified,-how far the variability of the actual experience depends upon imperfect observation, and how far it is a true record of differences in the thing measured. It is just this region of uncertainty, fringing our experience with a haze of doubt, which fascinates the real experimenter. $\mathrm{He}$ is always striving to reduce it within narrower limits, and to enlarge the region of experimental certainty as much as he can. I want to show you one case in which such an 
earnest effort to reduce the margin of experimental uncertainty led to an important and quite unexpected discovery.

A dozen years ago, Lord Rayleigh had a certain glass flask, which he weighed and measured very carefully, by means I cannot stop to describe. When he had done this, he weighed the quantity of oxygen this flask would hold at a temperature of $15^{\circ} \mathrm{C}$. and a pressure corresponding to a barometric height of $760 \mathrm{~mm}$. of mercury. Here are his final results. You see that if he had only kept four figures in his records, they would be in absolute agreement: but the fifth figure begins to show slight discrepancies.

Weight of Oxygen in a Flask at $15^{\circ} \mathrm{C}$. and $760 \mathrm{~mm}$. (Rayleigh).

$\begin{array}{lc}2.6272 & \text { grammes } \\ 2.6271 & , \\ 2.6269 & ", \\ 2.6269 & ", \\ 2.6271 & \text { ", }\end{array}$

In these records, each unit of the fifth figure means a ten-thousandth part of a gramme, which is a weight too small for any of us to perceive with our unaided senses ; it can only be detected by a skilful observer provided with a delicate balance. The differences between these results are therefore very small indeed; but the whole quantity measured is also small, and the range over which uncertainty is shown to extend is two or three parts in 20,000, a very much larger fraction of the whole result than is affected when we measure an angle of latitude, so that the accuracy attainable in the one case is much greater than in the other. This is due to the very complicated nature of the process by which oxygen must be 
prepared and weighed. It is impossible in the short time available to give you any adequate idea of the work involved in such a weighing. Most of you have probably not tried to weigh anything accurately. If you will try to prepare oxygen so that it is sensibly pure, and then to weigh a bottle of it at known temperature and pressure until your records agree to three figures, you will understand some of the reasons why there is a margin of uncertainty to our knowledge about the weight of a known volume of oxygen, and you will understand what it means to have reduced this margin of uncertainty within the narrow limits indicated by Lord Rayleigh's record.

When he had made this series of weighings, Lord Rayleigh tried to determine the weight of pure nitrogen contained in the same bottle at the same temperature and pressure : and some of the results he obtained are given here :-

Weight of the Nitrogen contained in a certain Flask at ${ }^{\circ} 5^{\circ}$. and $760 \mathrm{~mm}$. Barometric pressure. (Rayleigh.)

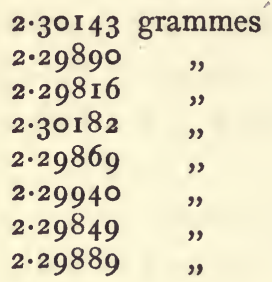

$2 \cdot 31017$ grammes

2.3100I

$2 \cdot 31024$,

2.31010 ,

$2 \cdot 31028$,

2.31035 ,

$2 \cdot 31026$,

$2 \cdot 31034$,

I have arranged these results in two columns. You notice that the numbers in each column agree with each other very well, though not so remarkably well as those obtained for oxygen : but any number in one column differs from those in the other by about one-hundredth of a gramme; and to a skilful physicist such a difference 
between successive weighings of the same volume of the same gas seemed altogether outside the possible range of errors in weighing. Lord Rayleigh therefore considered the possibility that the thing he had called nitrogen, and spent all this care in weighing, might not always be the same thing. Now the numbers in the first column were obtained by weighing nitrogen liberated from certain of its compounds: those in the second column, which are about one-half per cent. higher than those in the first, were obtained by weighing the gas left after a portion of atmospheric air had been deprived of its oxygen, its traces of carbonic acid and other known impurities, and its water. When this work was done, all chemists believed that the residue, left after treating air so as to deprive it of the constituents I have mentioned, was pure nitrogen: and the reason why they believed this affords us an instructive lesson in the care with which experimental discrepancies should be treated, before we neglect them. In the year 1785 , Cavendish published an account of his analysis of air. He found that in every hundred volumes of air there were nearly twenty-one volumes of oxygen, with a small quantity of carbonic acid, and he tried to find out whether the rest was pure nitrogen. His way of doing this was to confine the nitrogen in a glass tube like the tube of a barometer, the open end of the tube dipping into mercury, and to convert it into an oxide which would readily dissolve in a solution of potash floating on the mercury. If the whole volume of gas left after the removal of the oxygen and carbonic acid had been made up of pure nitrogen, and if Cavendish had been able to oxidize it all, the gas in his tube should have been completely absorbed. The process by which the nitrogen was oxidized in this experiment took a very long time, and at the end there was a small bubble of 
gas remaining. Cavendish was not sure what this small bubble of gas was. It might have been introduced by accident during the manipulations, but he said that if this bubble represented some constituent of the atmosphere, which was neither oxygen nor nitrogen, the amount of it was about one volume to every hundred volumes of atmospheric nitrogen. For a whole century no one seems to have repeated Cavendish's experiment with such care as to make sure about this residual bubble. Other means were adopted for the analysis of the air, and since these did not show the presence of any gas besides oxygen, nitrogen, and carbonic acid, Cavendish's residual bubble was forgotten or neglected.

But Lord Rayleigh and Sir William Ramsay, when they took counsel together how they might account for the peculiarity of atmospheric nitrogen, remembered this bubble; and Sir William Ramsay determined to find out what it really contained. By a variety of ingenious methods we cannot stop to consider, he did find out. He found that about one volume in every hundred of the gas in the atmosphere which had been regarded as pure nitrogen was not nitrogen at all, but was formed of a group of elementary gases, the chief of which is that called argon. Argon is about one-and-a-half times as heavy as nitrogen, so that the presence of about one volume of argon in every hundred volumes of the gas called atmospheric nitrogen renders this gas about onehalf per cent. heavier than pure nitrogen, and so accounts for the discrepancy between Lord Rayleigh's two groups of weighings.

The discovery of argon was, therefore, directly due to a refusal to replace the variable and discordant experience of the weight of nitrogen by an ideal uniformity based on the mean of the actual experiences. If Lord Rayleigh had 
replaced all the results in his table by a single compromise between them, and had been content to stop there, we should not know the existence of argon to-day. On the other hand, if the chemists of the nineteenth century had paid more attention to the discrepancies in their own experience of nitrogen, if they had not been content to replace their experience and that of Cavendish by a compromise which neglected his residual bubble, argon must have been discovered long ago.

Just in the same way, the slight changes in the position of the Earth's axis, by which the latitude of a telescope is changed, not by a change in the position of the telescope but by a shifting of the Equator,-these changes have only been discovered by examining all the records of experience, and refusing to replace discordant observations by an imaginary uniformity.

As it is in the two cases we have spoken of, so it is in others. The ideal description of every experience, the description which alone makes further progress possible, is a description of all the results obtained, and not a statement which largely ignores the inconsistencies observed. The reason why astronomers, and physicists, and chemists can so often afford to neglect the inconsistencies of their experience without making themselves ridiculous is that by great labour they have already succeeded in confining the limits within which these inconsistencies occur, so that the proportion of the whole experience affected by them is very small. But biologists have not yet advanced so far as this: the margin of uncertainty in their experience is still so large that they are obliged to take account of it in every statement they make. I tell you that the distance from the Radcliffe Observatory to the Equator is 6,300,000 yards, and you do not care whether the next measurement makes it a 
dozen yards more or less; the difference is too small to matter for most purposes. But if I tell you that Englishmen are 5 feet $7 \frac{1}{2}$ inches high, you remember your father who is five feet ten, and your cousin who is over six feet, and you think I am talking nonsense. The discrepancies between various observations of the height of Englishmen are so great that we cannot replace them by a single statement of this kind without immediate and palpable absurdity. If we want to make a statement about the stature of Englishmen, we must find a way of describing our whole experience; we must find some simple way of describing our whole experience, so that we can easily remember and explain to others how many men of any given height we find among a thousand Englishmen. We must give up the attempt to replace all our experiences by a single average value, and try to describe the whole series of results our observation has yielded. I want to show you how this can be done, and in order to show you, I will choose a kind of experience in which the uncertainty affects not merely a part of the result of any single observation, but the whole of it.

Suppose I take twelve dice and toss them. I do not know how many will show me more than three points in any one trial; none of them may show me more than three points, or one, or two, or any number up to the whole twelve may do so. If I make only a few tosses, I can find no sort of regularity among the results; but as the number of trials increases, I find that the frequency with which each of the thirteen possible results occurs approaches a constant and predicable percentage of the whole number. With my wife's help I have made three independent series of such tosses, each series containing $2^{12}=4,096$ trials, and here are the results :- 
Results of Tossing twelve dice $\mathbf{2}^{12}$ times.

\begin{tabular}{|c|c|c|c|c|}
\hline $\begin{array}{l}\text { No. of } \\
\text { Dice with } \\
\text { more than } \\
3 \text { points. }\end{array}$ & $\begin{array}{c}\text { First } \\
\text { Series. }\end{array}$ & $\begin{array}{l}\text { Second } \\
\text { Series. }\end{array}$ & $\begin{array}{l}\text { Third } \\
\text { Series. }\end{array}$ & Theory ${ }^{1}$. \\
\hline I 2 & $\mathbf{I}$ & 0 & $\mathbf{I}$ & $I \cdot 2$ \\
\hline I I & 13 & 8 & I 4 & I $4 \cdot 3$ \\
\hline Io & 86 & $6 I$ & 66 & $76 \cdot 1$ \\
\hline 9 & 246 & $24 I$ & $24 I$ & $244 \cdot 6$ \\
\hline 8 & 540 & $5^{\mathrm{I}} 3$ & 568 & 530.9 \\
\hline 7 & 836 & 856 & $86 I$ & 819.6 \\
\hline 6 & 913 & 948 & 866 & $922 \cdot 2$ \\
\hline 5 & $75^{\circ}$ & 802 & 728 & $762 \cdot 5$ \\
\hline 4 & 446 & 420 & 474 & $459 \cdot 7$ \\
\hline 3 & 198 & 182 & 204 & $197 \cdot \mathrm{I}$ \\
\hline 2 & 55 & $5^{I}$ & 67 & $57 \cdot 0$ \\
\hline I & 12 & I 3 & 6 & 10.0 \\
\hline 0 & 0 & I & 0 & 0.8 \\
\hline
\end{tabular}

You see that in each series of trials the individual results occur with a frequency in rough agreement with that given in the fourth column of the table. We cannot predict the order in which these results will occur, but we can make at least a rough prediction of the relative frequency with which any one of them will occur, in a sufficiently long series of trials. We can predict that one result will occur more frequently than any of the rest, and we can predict the way in which the other possible results will group themselves around it.

We cannot replace our variable experience of the results obtained from individual throws of dice by a statement of an ideal and constant experience, without getting into serious difficulties, because the margin of uncertainty is too great; but we can describe the whole series of

1 No dice are symmetrical. The entries in this column are the terms of $4,096(0.509+0.491)^{12}$, based on the observed mean chance of throwing more than 3 points with the dice used. 
experiences, with very fair accuracy, by a theory which enables us to predict the general characters of any long series of similar experiences.

Now, if we attempt to describe our experience of any character of living things, we find the same difficulties with individual cases, the same regularity when we deal with large series of cases, as we find when we attempt to describe the results of games of chance. In each case we are unable to predict the result of any single observation, before we have made it, but we can predict, with very considerable accuracy, the result of a long series.

The first important difference between the methods used by most astronomers, by most chemists and physicists, and the methods which ought to be used by students of biology, is determined by this fact that while astronomers, physicists, and chemists have by great and successful efforts reduced the limits of uncertainty concerning the results of their observations until for many purposes they can neglect the discrepancies between the results of individual experiments, and treat their experience as uniform, biologists have not yet gone so far, and they are still forced to base such general statements as they can make on the characters of long series of observations.

The method by which long series of variable results can be described, in such a way that the mind can easily remember and form a useful picture of each series as a whole, is provided by the science of statistics, and the application of these methods to biological problems, commenced by Quetelet, has been enormously extended of late years by the work of Francis Galton and Karl Pearson. The details of the method are logically unimportant, and I shall not trouble you by asking you to consider them, except in so far as it is necessary to do so in order to put before you one of the generalizations 
concerning inheritance which have been reached by its means.

All experience, which we are obliged to deal with statistically, is experience of results which depend upon a great number of complicated conditions, so many and so difficult to observe that we cannot tell in any given case what their effect will be. Consider the height of a man. This is a result depending on the length of each of a very large number of bones, cartilages, and other structures, and the length of each of these is determined in its turn by a large number of conditions, among which are the length of the corresponding part in the father and in the mother. When you know the height of a man's father, you know one out of a number of sets of conditions which have helped to determine the height of the man himself, but you are still in ignorance of the rest : and the student of inheritance is always in this position, that he is trying to find out how much he can learn to predict about results, when he knows only a few of the conditions on which these results depend.

In order to show you the kind of knowledge one can obtain in this way, and the limits to its exactness, I must ask you to let me trouble you once more with dice. I have here two dice, one white and the other red. Suppose I want to get more than three points with each when I throw them. I throw them, and try. I get a certain result. If I throw them both a second time, I am no more and no less likely to get more than three points with each of them than I was the first time, no matter what the result of the first throw may have been. The result of the second throw is quite independent of the first result.

Now I want to make the result of the second throw partly dependent on the first, and I do so in this way. s. 2 
After making my first throw with both dice, I pick up only the white die, letting the red pass over into the second throw, exactly as it was when it affected the result of the first throw. Here I make a first throw, and it happens that both the dice show more than three points; the red one shows four, and the white one six. That means that one of the dice I shall count in my second throw will certainly show more than three points, and all my uncertainty about the second result is due to my ignorance of what the other will show me. I now pick up the white die and toss it again, it shows two points only, so that for what I call a second result I have one die with more than three points, and one with less.

Just consider for a moment how much the knowledge, that one of the two elements on which the result of the first throw depended will pass unchanged into the second throw, has diminished my uncertainty about the result of the second throw. Whenever I throw two dice, both of them may show more than three points, or only one of them, or neither, may do so. A simple calculation shows you that if I make a long series of such throws, throwing both dice every time, both the dice will show more than three points about once in four trials, one of them only will show more than three points twice, and once in four trials both the dice will fail to show the points I am looking for. But if I know that both my dice showed more than three points in the first throw, and if I know that one of these will pass unchanged into the second throw, then I am sure that one of the two elements on which my second result depends will give more than three points, and my only uncertainty about the second result arises from my inability to predict what the second die will do. The single die I toss again to form my second result will show more than three points about 


\section{AND PLANTS}

once in every two throws; so that if I know that both dice in the first throw gave more than three points, I may expect that both of them will do so in a dependent second throw once in two trials, instead of once in four.

Now those of you who know Weismann's theory of the mechanism, if I may use that word for want of a better, by which characters are transmitted from parent to offspring, will recognize that the relation I have established between my first and my second result with these dice is roughly analogous to the relation he indicates between parent and offspring.

Weismann supposes that the characters, which a parent can transmit, are represented in the young germ-cell, whether male or female, by a certain number of elements, roughly comparable with the dice I have used. Before a germ-cell becomes ripe, so that it can fuse with another germ-cell of opposite sex, half the elements it contains are thrown out of it by what Weismann considers to be a purely random selection; so that if the constitution of a young germ-cell be represented by the result of a first throw of my two dice, the random removal of half its determinant elements will be represented by the removal of the white die. When a ripe germ-cell fuses with another of opposite sex, a number of elements, equal to the number it has lost in the process of maturation, is restored to it, and the characters of the embryo are determined by these two sets of elements, one set chosen at random from a young germ-cell of each parent. In making my second result with the dice, I have taken one element at random from the two which determined the first result, this process being roughly analogous to the loss of half the determinants during maturation, and I have restored the original number by adding to the one element which remains from the first result a second 
element taken at random; this addition is roughly analogous to the addition of elements during fertilization; so that the relation I have established between my second result and my first is roughly analogous to that which Weismann's theory leads us to expect between a child and one of its parents. It is still more closely analogous to the relation indicated as a result of direct statistical study of inheritance by Mr. Francis Galton.

I mention this to show you that the illustration of heredity by such a rough experiment as I have described has more justification than some of you may realize at first.

The experiment itself must be repeated many times, and on a larger scale, in order to illustrate the points I want to show you. With my wife's help, this has been done. She and I took not two only but twelve dice, and painted six of these red. Tossing all the twelve, we counted the number which showed more than three points, and so obtained a first result. The six red dice were then left undisturbed, the white dice were thrown again, and we had a second set of twelve, six carried over unchanged from the first throw, and six in a condition independent of that observed after the first throw: the number of dice with more than three points in this set of twelve gave us our second result. In this way we obtained a pair of results, half the elements which determined either member of the pair being common to it and to the other member. When we had obtained such a pair of correlated results, we tossed all the dice thoroughly, so as to destroy any effect of their previous arrangements, and went through the whole process again, until we had a second pair. We did this $2^{12}=4,096$ times, until we had 4,096 pairs of results, each result determined by some elements common to itself and to the other member of 
the pair to which it belonged, but quite independent of any other result in the series.

The results of our experiment are entered in the table

Result of First Throws $\rightarrow$

\begin{tabular}{|c|c|c|c|c|c|c|c|c|c|c|c|c|c|c|}
\hline & 0 & I & 2 & 3 & 4 & 5 & 6 & 7 & 8 & 9 & 10 & I I & I 2 & Totals \\
\hline I 2 & & & & & & & & & & & & & & 0 \\
\hline I I & & & & & & & $\mathbf{I}$ & I & 5 & $\mathbf{I}$ & & $\mathbf{I}$ & & 9 \\
\hline Io & & & & & & 2 & 6 & 28 & 27 & r 9 & 2 & & & 84 \\
\hline 9 & & & & $\mathbf{I}$ & 2 & I I & 43 & 76 & 57 & 54 & I 5 & 4 & & 263 \\
\hline 8 & & & & 6 & I 8 & 49 & I 16 & I 38 & I I 8 & 59 & 25 & 5 & & 534 \\
\hline 7 & & & & 12 & 47 & 109 & 208 & $2 I_{3}$ & 118 & 71 & 23 & I & & 802 \\
\hline 6 & & & 9 & 29 & 77 & 199 & 244 & 198 & 121 & $3^{2}$ & 3 & & & 912 \\
\hline 5 & & 3 & I 2 & $5 x$ & II 9 & I $8 I$ & 200 & I 29 & 69 & I 8 & 3 & & & 785 \\
\hline 4 & & 2 & r 6 & 55 & 100 & I I 7 & 9 I & 46 & 19 & 3 & & & & 449 \\
\hline 3 & & 2 & I 4 & 28 & 53 & 43 & 34 & 17 & I & & & & & 192 \\
\hline 2 & & & 7 & 12 & $\mathbf{I} 3$ & I 8 & 4 & $\mathbf{I}$ & I & & & & & $5^{6}$ \\
\hline $\mathbf{I}$ & & & 2 & 4 & $\mathbf{I}$ & 2 & $\mathbf{I}$ & & & & & & & 10 \\
\hline 0 & & & & & & & & & & & & & & 0 \\
\hline Totals & 0 & 7 & 60 & 198 & 430 & $73^{I}$ & 948 & 847 & $53^{6}$ & 257 & $7 I$ & I I & 0 & $4, \circ 96$ \\
\hline
\end{tabular}

Correlation between successive Throws of twelve Dice.

I now ask you to look at. It is just the kind of table you get when you describe the relation between parental and filial characters, so it is worth a little attention. You see 
that the table has 13 columns, numbered in order from $O$ to $I 2$, and 13 lines, numbered in order in the same way. The result of every first throw of a pair was entered in a column, the number of the column showing the number of dice with more than three points in the result recorded; those cases, for example, in which only one of the dice in a first throw gave the number of points recorded are entered in the column labelled $\mathrm{I}$, and so on. The number of dice which gave the points we were looking for in the second result of a pair determined the line in which an entry was made. In the table as it is printed, the entries in every square formed by the intersection of a line and a column are added together, and the totals only are given; thus in the square common to the line labelled 5 and the column labelled $\mathrm{I}$ the number 3 is entered, showing that there were three pairs of throws in which the first result was that one die gave the number of points sought, while in the second result five dice did so. Looking through column I, we see that a first throw which yielded only one die with more than three points was twice followed by a second throw containing three successful dice, twice by a second throw containing four, and three times by a second throw containing five.

The sum of all the entries in a column gives the whole number of first results of a particular kind; so that if you look at the sum of all the entries in the column labelled I I, and see that this sum is II, you know that out of the whole 4,096 first throws, there were II in which eleven dice showed more than three points. Similarly, the sum of the entries in each line shows you how often a second throw gave a particular result; in the row labelled I you find Io entries, showing that out of the 4,096 second results there were ten in which only one die gave more than three points, and by looking at the columns in which 
these ten entries are distributed we can see the character of the first throw which each of them followed.

Without much trouble you can see that a low number of successful dice in either a first or a second throw is generally associated with a low number in the other, and

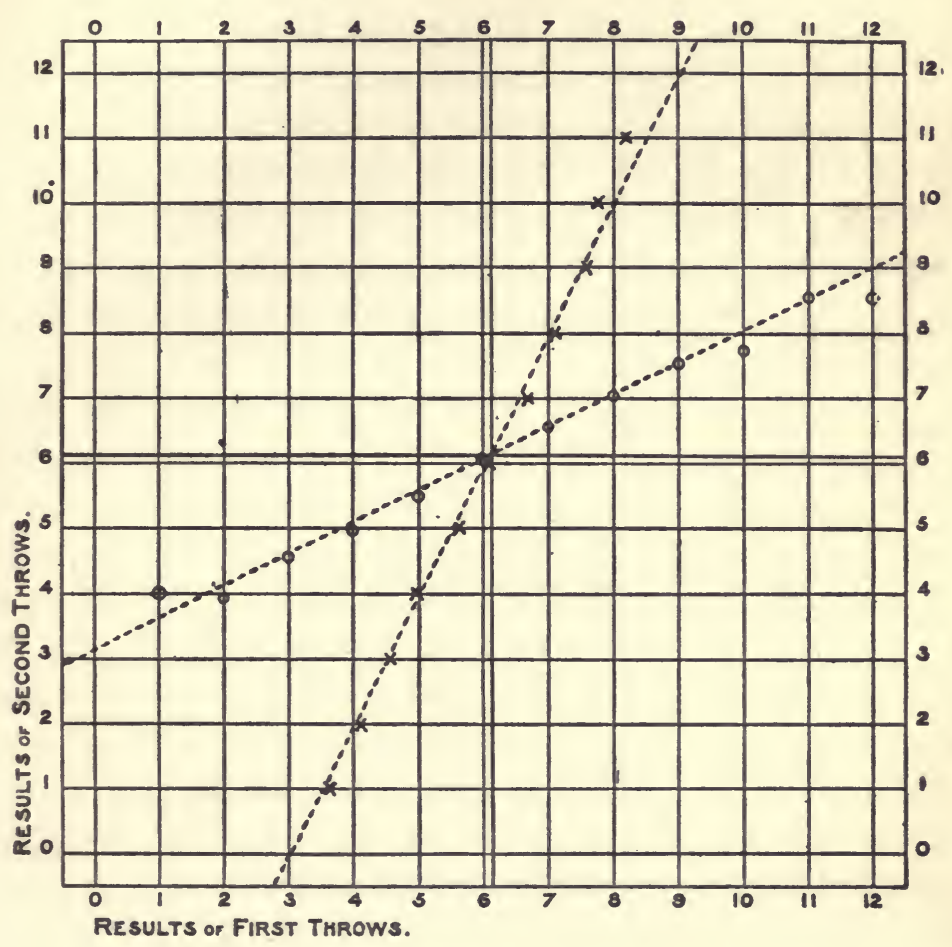

FIG. I.

a high number in one with a high number in the other. In line I I, recording second throws with II successful dice, all the entries are in columns which tell us that the preceding first throws had contained from 6 to. II successful dice, while in row I the entries are in columns numbered from 6 to 2, and tell us that when the second 
throw gave only one successful die, the number of successes in the preceding first throw was never more than 6 .

You see, then, that first results, which deviate from the average in either direction, are generally followed by second results, which deviate from their average in the same direction. I want to show you how we can measure the relation between first and second throws a little more closely; and for this purpose we will consider only the mean values of the entries in every row and in every column. It would be perfectly easy to consider the whole series of numbers as they stand, and students of heredity are obliged to consider quite similar series; but to do so would take too long.

In the diagram fig. I, every cross shows you the mean value of all the entries in a line of the table, every circle shows you the mean value of all the entries in a column. Every circle therefore shows you the average character of those second throws which followed first throws of a certain known character; every cross shows you the average character of those first throws which preceded second throws of known character. You see that each series, whether of circles or of crosses, lies fairly well upon one of the two dotted lines in the diagram. Be good enough to look first at the series of circles, and to begin by looking at the middle of it ; that is, consider the average results of those second throws which have followed first throws yielding six dice with more than three points, or first throws of average character. You see the position of the circle in the middle column shows that the second throws which followed these average first throws were themselves on the whole of average character, containing six successful dice. If you look at the cross in line 6, you find in a similar way that the first results, 
which preceded second results of average character, were also, on the whole, average. The circle of the sixth column, and the cross of the sixth line, lie almost one upon the other. Therefore, when either member of a pair gives an average result, the other member in the long run does so too.

Starting from the average in either direction, we may call the difference between any given result and the average of all of them, the deviation of that result. So, the average number of dice with more than three points in a first throw being 6 , when we find 9 dice with more than three points in such a throw, we may call the deviation of that result 3. Now the circle in the column 9 is very nearly indeed half-way between lines 7 and 8, or at the position $7 \frac{1}{2}$, showing that first throws which contained 9 successful dice were followed by second throws which contained on an average $7 \frac{1}{2}$ such dice. The deviation of these second throws from the average 6 is $\mathrm{I} \frac{1}{2}$, or half the deviation of the first throws which preceded them : and if you look carefully at all the circles, you will see that each of them is very nearly twice as far from the position which represents the average of all first results as it is from that which represents the average of second results. So that if we take any set of first results, whose deviation is the same in direction and in amount, the mean deviation of the associated second results will be half that of the first. If we know that a first result has given two dice more or less than the average number, with the points sought, the most probable prediction we can make about the associated second result is that it will give one more or less than the average number of successful dice.

This quantity $\frac{1}{2}$, which expresses the ratio between the mean deviation of a series of second results, and the known deviation of the first results which preceded them, 
is what Mr. Galton has called the 'Regression' of second results on first results. We may express the facts illustrated by the line of circles on our diagram by saying that the regression of second throws on first throws is $\frac{1}{2}$. If you examine the line of crosses as we have examined the line of circles, you will easily see that the regression of first throws upon second throws is also $\frac{1}{2}$.

If we had made our second result depend entirely upon the first, if we had carried all twelve dice unchanged from the first result into the second, simply counting them over again, without tossing them at all, the two results of each pair would have been identical, and if we tried to indicate that on such a diagram as fig. I, we should have to make the circles and the crosses of that diagram all lie on one straight line, inclined to the axes at an angle of $45^{\circ}$. The regression of either set of results upon the other would then be unity. If, on the other hand, both results had been entirely independent, the line of circles would become horizontal, the line of crosses vertical, and the regression of either first or second results upon the other would be zero.

I have been anxious to show you this phenomenon of regression first of all in an experiment with dice, because you see more clearly from such an experiment, the way in which it is brought about. You see that we are obliged to express the relation between our correlated sets of results with dice in terms of this kind, because when we know the result of a first throw, we know some of the elements which determine a second throw, but not all of them. There is nothing peculiarly vital in this phenomenon of regression, and I have been anxious to make that point clear to you, because it is not generally understood. The term Regression was introduced into biological work by Mr. Galton, in his discussion of the relation between the stature of human parents and the stature of their children. 
In his statements about regression, Mr. Galton always dealt with the phenomena of inheritance by a beautiful application of the mathematical theory of chance, which he was the first to use in treating biological problems : but very few of those biologists who have tried to use his methods have taken the trouble to understand the process by which he was led to adopt them, and we constantly find regression spoken of as a peculiar property of living things, by virtue of which variations are diminished in intensity during their transmission from parent to child, and the species is kept true to its type. This view may seem plausible to those who simply consider that the mean deviation of children is less than that of their fathers : but if such persons would remember the equally obvious fact that there is also a regression of fathers on children, so that the fathers of abnormal children are on the whole less abnormal than their children, they would either have to attribute this feature of regression to a vital property by which children are able to reduce the abnormality of their parents, or else to recognize the real nature of the phenomenon they are trying to discuss.

Our business to-day is with methods rather than with results. I will therefore only show you one example of regression between parent and child, and I choose the relation between the breadth of span in mothers and in daughters, which has lately been determined by Professor Pearson. Without giving you any more detailed numbers, the circles on the diagram (fig. 2) will show you how nearly the relation between the mean span of daughters and the known span of mothers is given by a regression line with the same slope as that giving the relation between the first and second results in our experiment with dice. The regression of second throws on first throws of dice is very nearly exactly $\frac{1}{2}$. Professor Pearson's value for the regression of daughter's span upon mother's span is 0.46 . 
I hope I have succeeded in showing you something of the special difficulties associated with the study of inheritance, and in making clear to you one of the methods by which experience of hereditary phenomena must be recorded. I will only add a word to show you the kind of general statement to which work of this kind seems to lead. It seems likely that in cases where the mating of parents is not determined to any serious extent by their likeness or unlikeness in the character discussed, the regression of children on parents has a value very nearly the same, and very nearly equal to $\frac{1}{2}$, for a large series of characters, mental as well as physical, in human beings, and for a large series of characters in the higher animals, at all events, if not in animals generally. It seems therefore likely that we shall soon be able to know with some accuracy how much we can predict about any group of unborn animals from a mere study of one parent. By such a study, we learn some of the conditions on which the characters of the young depend: if we examine both parents, we learn more of these conditions ; if we study not only the parents but the grandparents we learn still more; and so on. The results so far achieved make it probable that Mr. Galton's original prediction will be verified for the large class of cases to which he intended it to apply, and that the influence of the different generations of ancestors, as measured by the regression coefficients between these and existing individuals, will be found to diminish with the remoteness of the ancestors, according to the terms of a simple geometric series, which is sensibly the same at least for all those characters among the higher animals which have been properly examined.

I should transgress the limits prescribed for me if I said any more about the results which seem already within reach : fascinating as the subject is, I must leave you to picture to yourselves, as you will easily do, the important 
consequences which may follow from such knowledge as is promised: I can only hope that I have helped you to realize a little of the difficulty which besets this form of experimental inquiry, and the way in which some part of this may be met and overcome.

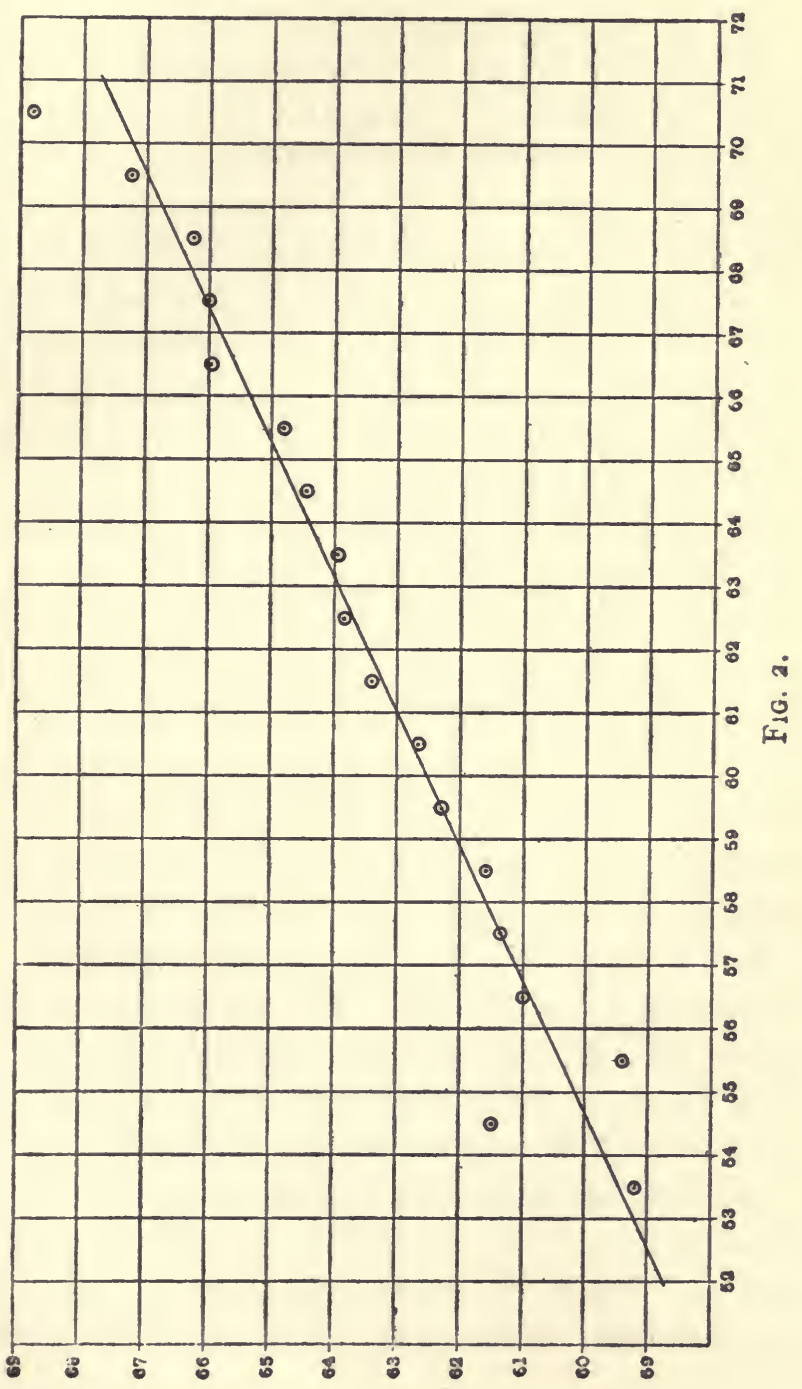




\section{V \\ PSYCHO-PHYSICAL METHOD \\ (W. McDougall)}

IT is my business to illustrate for you the application of the scientific method to that highly specialized and difficult branch of science known as psycho-physics. I propose to do this by defining one particular psychophysical problem, typical of many psycho-physical problems, and showing you the difficulties in the way of its solution and the manner in which these difficulties may be, in large part at least, overcome.

But it is perhaps desirable that, before I do this, I should say something of the nature of psycho-physical science in general, of its scope and aims, and of its relations to other branches of science. This is necessary because, unlike most of the departments of science that you will hear discussed in this course of lectures, psycho-physics is a very young science. It came into existence hardly half a century ago, and therefore is still somewhat amorphous; its boundaries cannot be sharply drawn, its methods are still in process of formation, and even its most fundamental conceptions are in an unstable state and are the subject of perpetual discussions which reveal wide differences of opinion among the workers.

It is for these reasons that, even in addressing a cultured audience such as this, it is desirable to attempt 
to define briefly the scope and aims of the science; especially in this country, in which this branch of science has not been pursued with the same ardour and has not attained to the same degree of popularity as in some other countries, notably in Germany and in America.

In the second lecture of this course, Professor Gotch put before you in glowing language that scientific conception of the universe to which the researches of the great physicists and mathematicians have led us; the conception of a universe consisting of matter and ether in ceaseless motion; a universe in which all events, the smallest and the greatest alike, are to be regarded as phases in the motion of matter and ether, resulting from, or wholly caused by, the preceding phases of the motion of matter and ether according to rigid unchanging laws, laws discoverable and in part already discovered by the application of scientific method to the study of phenomena. And Professor Gotch, like the majority of scientific men, did'not hesitate to include within this great scheme of things the bodies of animals and men and all the phenomena presented by them to our senses.

This conception is one that fascinates by its simplicity, and by the grandeur of its comprehensive sweep. But is this great conceptual scheme all-inclusive? Has science no other task than to fill in its details? Is there no province, no form of existence, that does not fall within its scope and that is yet capable of being studied by the scientific method?

There are some who to this question reply, 'Yes, there is no such province; when, or if ever, the physical universe shall be completely described in terms of matter and motion science will have achieved its task, and 
the universe will have been rendered completely intelligible.'

This view, first clearly propounded in ancient times by Democritus, flourished considerably in the middle part of the last century. But few scientific men will now subscribe to it. You will have noticed that Professor Gotch claimed only that this great scheme of things is that which scientific men have evolved in aiming to render intelligible the phenomenal world by means of the principle of physical causation. In saying that, he marked off, as falling within this scheme, one great group of sciences, the sciences that deal with the world of phenomena; with the things that we apprehend by means of the senses, the things we see and hear and touch and taste; with the objective world, in short.

But it is obvious that there is known by each of us another order of existence, a world of events not apprehended by means of the senses, a world not phenomenal and not objective in character, but subjective, the world of his own immediate experience, made up of his sensations and ideas, his pains and pleasures, his emotions and his strivings, and all the ever-varying states of his consciousness.

That great scheme, by means of which the physicist endeavours to render intelligible the phenomenal world, is reached by inference from the changes of phenomena to underlying causes and conditions, the properties, the motions and the collocations of matter and ether, the sum of which is conceived as making up the whole physical universe. Clearly, therefore, in that scheme there is no place for these facts of immediate experience, these states of our own consciousness. The universe we know seems, then, to consist of two orders of things, two orders 
of events; on the one hand the world of matter and motion, and on the other the world of consciousness, the physical and the psychical worlds.

Are we then to exclude the study of the facts of immediate experience, the states of our own consciousness, from the realm of science?

At one time this was the prevalent view and practice; the physical world was regarded as the exclusive object of scientific study, and the realm of psychical events was the field of metaphysics, i.e. the study of that which is not physical. So that we had an opposition of physics, or science on the one hand and metaphysics on the other.

But this usage of the term metaphysics, and this exclusive application of the term science to the study of the physical world, has now passed away. It is recognized that a branch of study should be classed as a science, not in virtue of the nature of the things with which it is concerned, but rather in virtue of the method by which it pursues knowledge.

Shortly after Bacon and his followers, the founders of the Royal Society, had established physical science on an empirical basis, by applying the scientific method of observation, induction, deduction and verification, John Locke, one of the brightest ornaments of this University, being imbued with the same scientific spirit, introduced this same empirical method into the study of that other great realm, the psychical world, and so carved out from the body of speculative opinion the science of mind or empirical psychology. Then for nearly two hundred years these two great orders of existence, the physical and the psychical, continued to be studied by the scientific method, but independently of one another, as though the two realms, the 
physical and the psychical, were in no way connected or related.

But, although science treated them as unrelated, it had long been clear to all men that the two orders of events, the physical and the psychical, are in some way intimately related. Nothing is more certain or more obvious. A solid body strikes sharply against your hand and you at once experience pain, a ray of sunlight falls on your face and you at once experience sensations of light and warmth, or you feel hungry and at once stretch out your hand to take food, you feel pain or discomfort and you at once move your limbs in order to get rid of it. We observe, in fact, a constant concurrence or concomitance of events of the two orders, the physical and the psychical, and this constant concomitance leads even the most unreflecting man to assume some orderly relation between them.

The fact of the relation has therefore always been recognized since men first began to reflect. But the nature of this relation, that so clearly obtains between the physical and the psychical worlds, remained a subject of speculation only until long after the scientific method had been applied with success to each of these realms independently. In fact, it was not until the middle of the nineteenth century that the scientific method was brought to bear upon the problem of the nature of this relation; and it was this, the application of the scientific method to this problem, that led to the development of that youngest branch of science known as psycho-physics.

For psycho-physics may be broadly defined as the application of the scientific method to the investigation of the relation between the psychical and the physical.

This step was taken, and this new branch of science was founded, by Gustav Theodor Fechner, Professor of 
Physics at Leipzig, with full consciousness of the nature and importance of the step. In his celebrated work, Elemente der Psycho-physik, published in 1860, Fechner says, 'By psycho-physics is to be understood an exact study of the functional relations, or relations of dependence, between body and soul, or, in more general terms, between the bodily and the mental, the physical and the psychical worlds.' Fechner, who first used the name psycho-physics and who is justly called the father of the science, was led to undertake its foundation by the desire to establish by the scientific method on an empirical basis a certain view of the relation of the physical to the psychical world, a view which he had long held as an essential part of a speculative philosophical system.

Putting aside the extreme idealists, who would resolve the physical universe that each man seems to see about him into a construction of the mind that has no existence save in the mind, we may recognize two great and opposed doctrines of the relation of the physical to the psychical. These two took definite form in the seventeenth century, in the hands of Descartes and of Spinoza respectively. Descartes's doctrine was dualistic. $\mathrm{He}$ held that there are two fundamental substances or enduring realities, matter and spirit, or extended substance and thinking substance-that the two worlds constituted of these two substances are for the most part independent of one another, but that they come together, have points of contact or interaction, in the brains of men, this interaction giving rise to the conscious life of man.

Spinoza's doctrine, on the other hand, was monistic. He taught that there is only one enduring substance or ground of all appearances, and that this is revealed 
to us under two aspects, the physical or material and the psychical or mental. Everything has these two aspects, if we could but discern them. Hence everything, not only the animals and plants, but all those objects which we are accustomed to regard as inanimate and purely material, have, like ourselves, their inner psychical aspect as well as the physical aspect which alone we apprehend through the senses.

It was this doctrine of universal animation or pan-psychism that Fechner accepted as an essential part of his speculative system. He could not bring himself to believe that the whole beautiful world was a soulless mechanism. The commonly accepted view of science, which regards the physical universe as merely a swarm of atoms in never-ending motion, among which here and there little points of light, the consciousness of men, appear for a few brief moments, only to disappear once more, leaving the meaningless swarming of the atoms to continue through the ages - this view he called the Night-view of nature; and over against it he set what he called the Day-view of nature, the view that every part of the whole universe not only exists as atoms swarming in scattered groups, but has also the feeling of own its existence and a joy in its own activity, and that this consciousness of each part is but an element in the universal world-consciousness.

It is the peculiar distinction of Fechner that, having accepted this Day-view of the world on purely speculative, aesthetic and religious grounds, he was not content to leave the matter there, like all his predecessors, but had the immense courage to set out to prove it by the application of the scientific method to the investigation of the relation of the physical to the psychical. How exactly he hoped to prove the truth of his view 
I have no time to show you. And that is now a matter of historical interest only, because it is now generally admitted that he failed to find the proof he sought. The road proved longer, the task more arduous, than Fechner had hoped; and we realize now that generations of workers, even of workers as keen and industrious and as bold as Fechner himself, must pass away before the issue of this great dispute can be decided. We recognize that the work of psycho-physics, like that of all other branches of science, must involve a vast amount of patient, laborious investigation of minute problems, investigations which, taken individually, may well seem trivial, and which perhaps, even more than the detailed work of other sciences, are apt to seem to the vulgar utterly useless and therefore absurd.

But for some minds the work has this great fascination-that, however small the facts investigated may be, however useless the results may seem from the point of view of practical application, they all in some degree bear upon, and contribute towards the solution of, a problem which, though it lies altogether outside the scope and beyond the purview of physical science, is yet profoundly interesting, because its solution is essential for the understanding of man's position and destiny in the universe; I mean, of course, the problem of the relation of the physical to the psychical, the psycho-physical problem par excellence.

Though Fechner failed to establish his view, he achieved much in founding the science of psycho-physics. It had often been asserted that mental states can never be subjected to exact and quantitative observation, and some, holding to the false dictum that science is measurement, had drawn the conclusion that the study of the mind can never become a science. 
Fechner boldly set to work to devise methods by which exact observations can be made, for he believed that the proof he sought would be yielded by an exact determination of the quantitative relations of the physical to the psychical. In attempting this he naturally turned to those states of consciousness which are most clearly and immediately related to the physical world, namely, the sensations. He sought to discover exact quantitative relations between sensations and the physical impressions, the stimuli to the sense-organs, by which they are excited.

That sensations have in some sense magnitude, and differ from one another quantitatively, seems clear. That an intense sensation, a bright coloursensation or a loud sound, is greater than a feeble sensation, a faint colour or a feeble sound, seems indisputable; but when we attempt to measure these magnitudes and to express them in numerical form we meet with great difficulties.

All physical measurement involves the comparison of the quantity to be measured with some accepted standard or sum of units of quantity. When, for example, we measure a quantity of length or of weight, we find how many times some conventional unit of length or of weight is contained in the quantity to be measured, and we express the quantity as a multiple of this unit. And in physical measurement we can adopt various expedients for reducing the equality or difference of the quantities to be compared to some form easily and certainly apprehended by the senses. Thus, in measuring weight, we are not content merely to compare the unknown quantity directly with the known standard of weight; but by means of a balance we render the difference or equality visible to the eye, which apprehends equality or 
difference of position and length with great accuracy. In a similar way, in measuring temperature we do not attempt to effect the measurement by a direct comparison, but by means of the thermometer we cause the changes and differences of temperature to appear as changes and differences of position apprehended by the eye. Almost all refined physical experiment consists in converting small differences of quantity inappreciable by the senses into some kind of difference more easily perceptible.

But in attempting to measure psychical quantities these two great resources are denied us. We have no means of procuring at any moment a sensation of any particular intensity that might serve as a standard unit of quantity. And even if we could do this, there would remain the further difficulty that we could not take a number of such units and, by adding them together, make a sum of units equal to the quantity to be measured, as when in physical measurement we take a sum of inches on the foot-rule or a sum of ounces in the pan of the balance. Nor can we convert the intensities of sensation that we wish to measure into any more easily measurable quantities, as we do in the case of physical measurement.

Fechner, looking round for means of measurement, noted four ways in which measurement can be made :-

(I) The just perceptible sensation-a very feeble stimulus excites no sensation, and by then gradually increasing the strength of the stimulus we can note the quantity of stimulus that just suffices to produce a perceptible sensation; that is then the measure of the threshold of sensation.

(2) We can compare two sensations together directly, and note their equality or difference.

(3) We can directly compare degrees of 
difference between pairs of sensations. (4) We can note the quantity of difference between two stimuli that just suffices to produce a perceptible difference between the corresponding sensations.

All such judgements of likeness and of difference are, however, liable to be affected and rendered variable by very many factors, both physiological and psychological, and Fechner therefore, starting out with these four possibilities of measurement, proceeded to devise and elaborate methods by which this variability and uncertainty might be eliminated. The methods which he devised are known as the psycho-physical methods; they are indispensable for almost all exact psycho-physical experiment, and they have been applied with success in a great number of instances.

I propose to try to make clear to you presently the nature of one of these methods by illustrating its application to a particular problem, with the aid of a simple apparatus. But before doing that, I must say a little about the relation of psycho-physics to its allied sciences.

Fechner's great enterprise failed for this reason chiefly. He was attempting to establish quantitative relations between psychical magnitudes and physical magnitudes with which they are related, between intensities of sensation and the magnitudes of the physical stimuli that excite them. But we know now that this relation is not a direct one. Between the physical stimulus, say the ray of light of given intensity falling on the eye, and the resulting sensation of colour there intervenes a long chain of processes that take place in the senseorgan and the nervous system. The ray of light induces some chemical or physico-chemical change in the retina; this in turn induces some chemical or physical change in the optic nerve, which change is propagated 
along the optic nerve to the brain; and it is not until this change, this nervous-impulse as it is called, reaches a particular spot on the surface of the brain that the sensation springs into existence. If the nervous elements in this part of the surface of the brain are destroyed, or the nerve fibres connecting them with the senseorgan are interrupted in any way, the physical stimulus will fail to excite the sensation.

The relation between the sensation and the physical stimulus that excites it is therefore, although fairly constant and definite, very indirect; it depends upon the integrity of groups of nervous elements in the sense-organ and in the brain, and of a chain of elements connecting them together; and the immediate psychophysical relation is that between the sensation and certain nervous processes in the substance of the brain.

Before, then, we can obtain any deep insight into the nature of this relation, or can reach any such conclusion as Fechner sought, we must know far more than we at present do of these delicate and complex processes that go on in the brain and in the nerves and in the senseorgans. Hence psycho-physics allies itself intimately with the study of these processes, the physiology of the nervous system and sense-organs, and thankfully accepts all the facts that the physiologists have been discovering at a constantly increasing rate ever since Johannes Müller in Germany and Sir Charles Bell in this country set this branch of research on the path of rapid progress nearly a century ago. And it is not content to receive only from physiology-the application of its methods has thrown new light on many purely physiological problems.

With empirical introspective psychology, for so long pursued as an isolated branch of science, psycho-physics 
maintains also intimate relations. Here, again, it not only makes use of the analytic descriptions of mental states effected by introspective psychology, but it has given, perhaps as much as it has received, for its adoption of experimental methods has led to the extension of these methods to the investigation of problems that are not strictly psycho-physical, but rather belong to pure psychology; it has thus raised empirical psychology from the level of a science of simple observation to the plane of experimental science; and at the same time it tends always to bring a larger part of the domain of pure psychology within its own boundaries, by seeking out the nervous events that are most intimately related with the various psychical states, and exhibiting the correlation of events of the two orders.

Thus it mediates between these two sciences, bringing the data of each to aid in the interpretation of the data of the other; and so, by bringing about co-operation between them, it is doing away with what has been nothing less than a scandal, the antagonism between the students of the material organism and the students of the psychical processes.

It seeks the aid also of comparative psychology, which is now being studied with a strictness of method that makes it very different from the mere collection of anecdotes that for long was all we had in this department. And it is beginning to render intelligible the nature of the instinctive actions of animals, those actions which for so long have seemed to be enveloped in impenetrable mystery, and to enable us to bridge in a vague and tentative way the immense gap between the human and the animal mind.

Again, it is beginning to bring within its sphere the whole problem of animal and especially human evolution, 
for it is realized now by an increasing number of thinkers that the theory of organic evolution cannot be treated as a branch of physical science; that if, in considering this great problem, we leave out of account the part played by the mind, by its feelings, its desires, its power of choice and its efforts, we are making an undue simplification which will render impossible an approximation to a complete theory of evolution; that, in fact, the problem of animal evolution is distinctly a psycho-physical problem - $a$ fact recognized by Darwin himself in his doctrine of sexual selection and in his treatment of instinct.

Lastly, just because psycho-physics is the application of the scientific method to the problem of the relation of the physical and psychical worlds, a problem which necessarily is dealt with by almost every speculative or metaphysical writer, the psycho-physicist has to make himself familiar with all such speculations, he has to keep them in mind, and from time to time has to ponder the bearing upon them of the positive results achieved by his science.

We turn now to illustrate the application of psychophysical method to a particular problem.

The characters of a sensation excited by a stimulus depend not only upon the quality and the intensity of the stimulus, but also upon its duration, and the problem I have chosen for illustration is this-How does the intensity of a visual sensation depend upon the duration of the stimulus? How does it vary with it?

If a ray of light of given intensity is allowed to act on the retina for a brief moment only, the sensation it excites is much less intense than if it is allowed to act for a longer period; i.e. the light must act upon the retina a certain length of time in order that it shall excite its 
full effect in consciousness. Let us call this duration, necessary for the production of the full effect, the action-time of the light. This time is by no means extremely brief; it is many hundred times longer than the duration of a flash of lightning or of an electric spark. That fact I can easily demonstrate to you presently. What then is this duration in the case of a light of any given intensity? If we can find the answer to this question, other problems open up and can be solved by modifications of the procedure. But we will confine our attention to this first step of the whole problem before us, the variation of the sensation $x$ with the duration of the stimulus.

It was first attacked by Helmholtz fifty years ago. The plan he adopted was to take a disc of light, divided into two equal parts of equal physical brightness, and to expose both halves for a brief moment simultaneously to the eye of the observer, the duration of the exposure of the one half being slightly longer than that of the other half. Then so long as the duration of exposure of both halves is less than the action-time, the half which is the longer exposed should appear the brighter, for it has the longer time in which to produce its effects in the retina, and will therefore more nearly produce its full effect. If this is found to be the case, let the duration of exposure of both halves be increased step by step in successive experiments, until the difference of brightness no longer appears. Then we may assume that the duration reached when the difference of brightness ceases to be perceptible is approximately the actiontime of that light.

By this method a result was reached which, however, was far from a correct measure of the action-time. To what, then, was the error due? You may be sure that 
the error was not due to any defect in the construction or working of the apparatus. As we should expect, the physical conditions of the experiment were faultless.

But it is a peculiarity of psycho-physical experiment that in every case you have to satisfy conditions of three kinds, namely, physical, physiological and psychological conditions. Perfection of the physical conditions avails nothing if these other conditions are faulty. And by Helmholtz's procedure the physiological and the psychological conditions were not satisfied.

The physiological conditions were unfavourable because, as we now know, when two parts of the retina are simultaneously but unequally excited, there takes place an interference between the two nervous processes, and this results in modification of the intensities of the resulting sensations, the one being rendered more, the other less, intense, an effect which we call a contrasteffect.

The psychological conditions were unfavourable because, in order to effect an accurate comparison of the intensities of two sensations, it is necessary to turn the attention first to one then to the other, and this was impossible when the two momentary sensations were simultaneously excited.

The imperfection of the procedure illustrates a truth of great importance. It may seem to you strange that the genius of Helmholtz and his great experience and ingenuity in all kinds of optical experiment should not have enabled him to devise at once a perfect method. But every man, no matter how great his genius, is more or less dependent upon the state of science and of scientific method that has been worked out by his predecessors. And when Helmholtz devised these experiments the psycho-physical methods of experiment 
were in their earliest infancy. Fechner had not then published his Elemente der Psycho-physik.

Helmholtz's results were accepted and no material advance was made with the subject, in spite of the work of one or two observers, until after fifty years Professor Martius of Kiel arrived at a result very different from Helmholtz's by means of a modification of his method. The modification consisted essentially in this, that instead of exhibiting two semicircles of light simultaneously for comparison, he exposed side by side two circles of light of equal physical brightness. Of these one was fixed and enduring, the other of brief duration only and recurring at regular intervals of some seconds. The observer had then to compare the brightness of the momentarily appearing circle with that of the enduring circle, and the duration of the exposure of the former was increased step by step until it appeared to be equally bright with the latter. This duration was then accepted as the action-time of light of that particular intensity. And this was about one-tenth only of the figure for the action-time found by Helmholtz's procedure.

Was then this procedure faultless, and was Helmholtz's result so very far wrong as Martius would have it?

Martius' procedure is as perfect as Helmholtz's physically, and it satisfies better the psychological conditions, because the attention is turned directly from the one impression to the other. Again, it avoids the error due to contrast, but on the other hand a new flaw is introduced into the physiological conditions of the experiment. When a ray of light falls on the eye the sensation it excites is brightest in the first moment of its appearance, and declines very rapidly in brightness owing to fatigue of the nervous substance, so that even within half a 
second a very considerable decline takes place; and this decline affects one's judgement of the brightness of the total impression, although one may be unable to distinguish the phases of declining brightness. Now in Martius' experiments one of the two impressions to be compared was in each case one of considerable duration, i.e. it appeared less bright than it should have done, with the result that a duration was assigned to the action-time much briefer than its true duration.

We have, then, to devise a procedure which shall avoid all these three sources of error, and this seems to be accomplished by the aid of the simple apparatus that we have here-a rough imitation of the laboratory apparatus. A large disc, with two windows of variable angular width at opposite ends of one diameter, rotates at a constant rate in the focus of a ray of light from the lantern, and in the plane perpendicular to the path of the ray.

As each window passes across the ray the light passes through it, falls upon the screen, and is reflected to your eyes during a fraction of a second, whose duration is easily calculated when we know the rate of rotation of the disc and the angular width of the window; e. g. if the disc makes I revolution in 3 seconds, and the angular width of the window is $10^{\circ}$, then the duration of exposure and of the action of the light on your eyes is $\frac{3 \times 10}{360}=\frac{I}{12}$ sec. By altering the width of the windows and reading off their width on a scale of degrees, the durations of the alternate flashes may be modified to any required extent.

The first point I want to demonstrate is that a flash of very brief duration has not time to develop its full effect in the retina of the eye. We make the windows 
$2^{\circ}$ and $10^{\circ}$ in width respectively. The light of alternate flashes is then of the same physical intensity but of different duration, and you see that the flashes appear alternately brighter and less bright.

Now for the procedure to determine the action-time of light of this particular intensity and quality.

We make both windows so narrow that the duration of each flash shall be less than the action-time, which we first determine very roughly by trial and error. And we make one window just so much narrower than the other that the difference of brightness of the two flashes is just distinctly perceptible.

We then increase the width of both by small steps of equal proportional extent, until we reach such a width that the longer flash no longer appears the brighter.

We know then that the duration of the longer flash slightly exceeds the action-time; for as soon as the duration of the longer flash equals the action-time, a further increase of its duration no longer increases its brightness, whereas a similar increase of duration of the briefer flash still increases its brightness; therefore, when this stage has been reached, the just perceptible difference of brightness between the two flashes is abolished by a further small increment of duration of each flash.

Here we must note an important feature of psychophysical method by means of which we can further refine our determination of the action-time.

We cannot arrive at a certain conclusion as to the presence or absence of a just perceptible difference of brightness by a single comparison. The internal factors that affect the accuracy of our judgement are so many and so variable, that a difference of stimulus which at one moment suffices to determine a judgement of difference may fail to do so at another moment; e.g. the attention 
may be not fully prepared, or the accommodation of the lens of the eye may be imperfect at the moment of observation.

Hence, in order to refine our result we must repeat the comparison many times at each step of increase of width of our windows. We shall then find that, when the difference of brightness is very slight, we shall fail to detect it in a certain proportion of cases, while in other cases we do detect it. But so long as we truly detect the difference we shall find in a given number of judgements, say 20 , an excess of judgements of difference over judgements of no difference, and so we can go on diminishing the difference of brightness of the two flashes until the stage is reached at which this excess of judgements of difference is abolished.

By means of this procedure we reach a much more accurate determination of the action-time than is possible by means of a single comparison or of comparisons repeated two or three times only. This is known as the method of right and wrong cases. It was devised by Fechner to eliminate the uncertainties arising from the variability of judgement due to the many factors by which it is liable to be favourably or unfavourably affected.

This procedure seems, then, to be satisfactory. The physical conditions seem good, and we avoid the error due to continued exposure of one of the circles to be compared, and also that due to physiological interference or contrast; the psychological conditions are good because the successive flashes come at intervals of $1 \frac{1}{2}$ seconds, and that, as we know from experience, is an interval very favourable to exact comparison of any two impressions; lastly, by applying the method of right and wrong cases we still further refine the result. And the figure we find in this way lies about midway between the extremely divergent 
figures assigned as the measure of the action-time by Helmholtz and by Martius respectively.

I have not time to show you how, having devised what seems to be a satisfactory procedure, one may go on to determine a number of interesting points. But I would like to draw your attention to the fact that the results of these psycho-physical experiments have an important practical application.

Such experiments may seem to you very remote from any bearing upon practical life, and to the vulgar mind they might seem for that reason to be useless. Now, if they had no such bearing, that would be no reason for pursuing such researches with any less ardour. For all such results of experiment go to the building up of psychophysical science; they are essential elements of the complete structure, though they may be neither foundation-stones nor head-stones of the corners.

Nevertheless, it is a legitimate source of satisfactiona satisfaction of which no scientific worker need feel ashamed-when the results of researches, undertaken from the point of view of pure science, are found to have practical applications.

It is, therefore, interesting to note that these psychophysical experiments have an important bearing upon the work of the lighthouse-engineer. Engineers have recently realized that by reducing the duration of the flash of light sent out from a lighthouse down to the physiologically permissible minimum, they effect a great economy of luminous energy, i.e. they can with the same source of light, the same lamp, make the ray sent out penetrate to, and be visible at, a very much greater distance than if the flash is made of longer duration, because they concentrate a wide beam into a narrow pencil of intense light. 
Hence you find that in many lighthouses, especially those on the coast of France, where the engineers have taken up this improvement eagerly, the flashes of the revolving lights are very brief. But in order that the maximum economy and efficiency of the light may be achieved, the engineer must know just what we have called the action-time of the light, the duration of the flash necessary for the production of its full effect on the eye and the consciousness of the observer. This knowledge has only been put at the disposal of the engineers by the development of psychophysical method; and this development we owe entirely to the spirit of pure science which, embodied in its loftiest form in Fechner and his successors, set out to apply the principles of scientific method to the investigation of the relation of the physical to the psychical world: that great problem which for so long had remained as a subject of speculation only, but which is now being brought within the pale of science by methods of which I have tried to give you a glimpse in this lecture. 


\section{VI}

\section{THE EVOLUTION OF DOUBLE STARS}

\section{(A. H. Fison)}

IN the astronomical discovery of the last twenty years, an increasingly important position has been assumed by the phenomena of double stars. Double stars are so richly distributed over the heavens as to indicate that the cosmic process by which they have been developed is a common one and that it has taken place in all parts of the physical universe. In the present lecture I propose to describe the general results of some recent researches that appear to suggest what this process has been, and, although it is certain that the actual conditions existing in nature are far from being so simple as those it is necessary to assume as a basis for mathematical reasoning, we meet with so many features of systems of double stars that are in such admirable harmony with the deductions from theory, that it is impossible not to regard the theory as expressing in its essential features an important cosmical process.

The discovery of double stars followed as a necessary consequence of the invention of the telescope. It was soon found that there were many stars that, while seeming single to the naked eye, appeared in the field of the telescope as two stars close together. Such an appearance may obviously be due to either of two causes. The two stars may be really close together, in which case 


\section{THE EVOLUTION OF DOUBLE STARS 133}

the pair are known as a binary star, and binary stars are the only true doubles, or they may actually be very far apart, their seeming proximity being apparent only, and due to their lying by chance nearly in the same direction in space. In this case they are said to form an optical double, and have no interest in the present study. The two conditions are illustrated in figs. I and 2. If it were

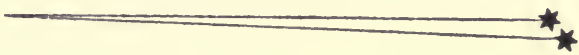

Fig. I. A binary star.

possible to measure the distances of the components of a double star from the Earth, it would obviously be possible to determine in any case to which of the two classes it belonged, but unfortunately it is only in rare instances that the measurement of the distance of a star is possible, and in no case has the application of this method to the

Fig. 2. An optical double.

examination of the binary character of double stars been of practical use.

There is, however, another way by which the binary character of a double star may be recognized. If the component stars are actually close together, and if the laws of motion and gravitation that control the movements of the bodies composing the solar system are also applicable to them, they must attract each other with a force varying inversely as the square of the distance between them, and each must in consequence describe an orbit round the other. The form of the orbit must 
be either an ellipse or some other of the conic sections, and each star must be in a focus of the orbit that the other describes relatively to it.

It is very important to realize clearly the nature of this motion. If we imagine a body, free to move and initially at rest, to be continually attracted towards a fixed centre, it will clearly move directly towards it, and, if the fixed centre is that of an attracting body, a collision is inevitable. If, however, the attracted body has an independent motion that is not directly towards or away from the centre of attraction, it will not move directly towards the centre of attraction, but it will pass round it in a conic section, and if, in addition, its independent motion is not excessive, the particular conic section traced will be either an ellipse or a circle, which last may indeed be regarded as an ellipse in which the longest and shortest diameters have become equal. This movement is almost exactly followed in the revolutions of the planets round the Sun.

So far we have assumed the centre of attraction to be fixed, but force is a mutual influence exerted between two bodies, and, in accordance with Newton's third law of motion, whatever force one body exerts upon another, this other must exert an equal and opposite force upon the first. Hence the two components of a double star exert equal forces of attraction upon each other, and both must move in consequence. The general solution of this problem is that the two bodies must describe similar conic sections round a certain point between them that is a focus of each orbit. This point is the centre of mass of the pair. If the speeds of the bodies are not excessive both orbits are either ellipses or circles. These laws were first established by Newton towards the end of the seventeenth century. 
A particular simple case of the problem is represented in fig. I2 (p. 162). $A$ and $C$ are two stars. In consequence of their mutual attraction and the motions that they possess independently of it, they describe similar orbits, which are in this case circles, about $O$, their centre of mass. If their masses were equal the point $O$ would lie midway between their centres, but in the case represented it is assumed that $A$ has twice the mass of $C$, and in consequence, the point $O$ divides the line joining their centres, so that its distance from the centre of $A$ is onehalf its distance from the centre of $C$. $A$ consequently describes a small orbit round $O$, while $C$, always lying upon the opposite side of $O$, describes a larger one. The case is precisely analogous to that presented by the Earth and Moon. Under the controlling influence of the Earth's attraction, the Moon is commonly regarded as describing a circular orbit round the centre of the Earth. This however is not a complete statement of the fact. The Earth attracts the Moon, but the Moon must necessarily attract the Earth with equal force. Consequently, both Earth and Moon describe orbits in the same time round their centre of mass. Since the mass of the Earth is eighty times that of the Moon, the centre of mass of the pair is quite close to the centre of the Earth, and the orbit described by the Earth is very small. In this case the orbits are not true circles, but are slightly elliptical. They are however precisely similar in form.

A case in which the ellipticities of the orbits are very pronounced is illustrated in fig. 4 (p. I 39). Here $S$ and $Z$ are two stars, describing, in consequence of their mutual attraction, similarly elliptical orbits round their centre of mass $O$. The mass of $S$ is twice that of $Z$, and consequently $O S$ is one-half of $O Z$. As the revolutions proceed the line $S Z$ continually passes through $O$, and 
the proportion between $O S$ and $O Z$ is accurately preserved. If the system were uninfluenced by any external body, $O$ would remain absolutely at rest. It is a focus of each of the two elliptical orbits.

If, in any case similar to those we have imagined, we were to select arbitrarily either of the two bodies, and if

\section{c. (1905).}

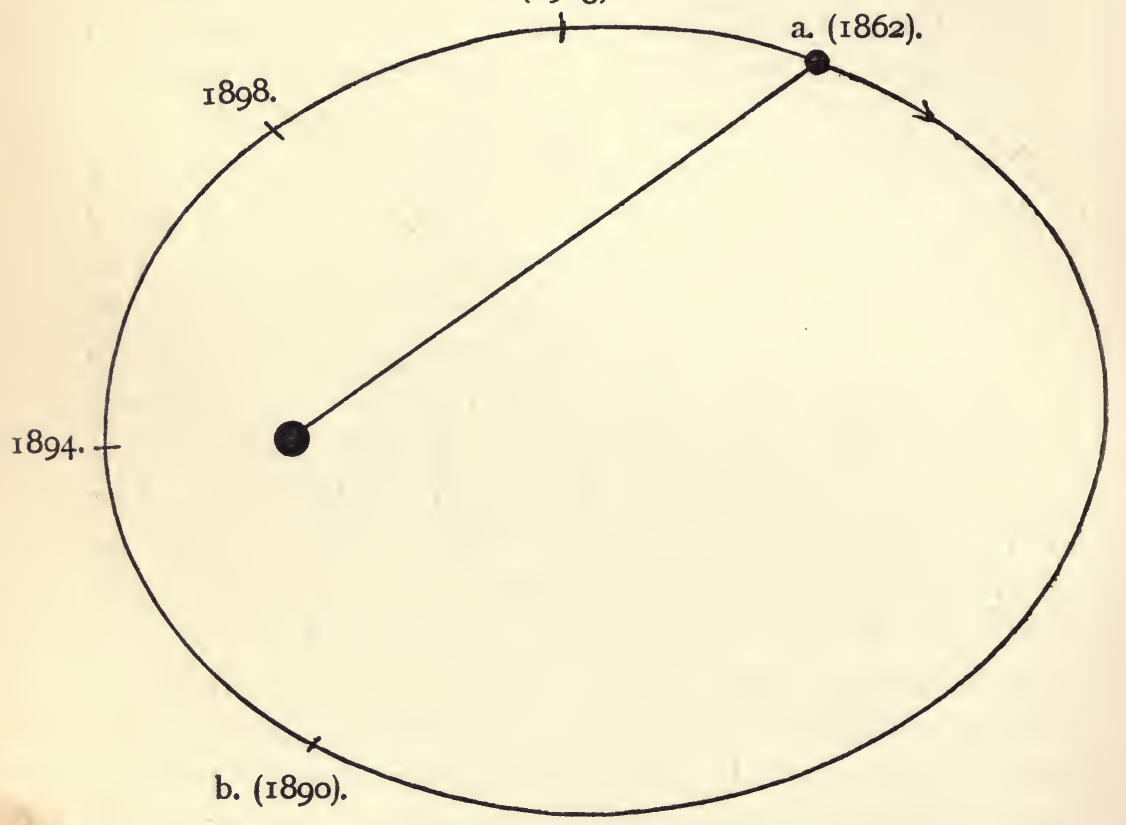

FIG. 3. Sirius, and relative orbit of companion.

we were then to measure the distance and the direction of the other from it from time to time, and if we were then to trace the orbit of the second body from these observations, assuming the first to be at rest, we should obtain a curve similar in form to either of the orbits, but larger. Its linear dimensions would in fact be the sum of those of the separate orbits. Such a curve is called the relative 
orbit of the second body. In the study of double stars it is, except in a very few instances, the only one that it is possible to obtain, and for many purposes it is as valuable as a record of the separate orbits. As illustrations of relative orbits the reader may refer to fig. Io (p. 160), in which the orbit traced is the relative orbit of the star $C$ in fig. I 2. Again in fig. 3 (p. I 36) the stars are those of fig. 4 , but the ellipse drawn is the relative orbit of $Z$. In comparing the corresponding figures it should be noticed that the same scale has been retained in the actual and relative orbits, and that the distances between the stars, as well as the directions in which either would appear if seen from the other, are the same whether the actual orbits of the pair, or the orbit described by one of them relatively to the other, are taken to represent the motion.

If then the components of a double star are in actual and not merely apparent proximity, each should, in obedience to the laws of motion and gravitation, describe an elliptical orbit (the relative orbit) round the other, or, more definitely, both should describe elliptical orbits round their centre of mass as a common focus. In our illustrations we have already anticipated that this mutual revolution is actually observed. It was first detected by Herschel in 1802 in the components of Castor, and at the present time several hundreds of mutually revolving pairs are known. It will be sufficient to illustrate these by the detailed description of one case, that of Sirius.

In 1862 a small star was detected by Alvan Clark in the immediate neighbourhood of Sirius. Observations have since shown that it is in motion, following an elliptical path round Sirius, and from repeated measurements of its distance and direction from Sirius it has been possible to trace its relative orbit with considerable 
accuracy. It is actually represented in fig. $3^{1}$. The period of revolution is fifty-two years. When first discovered the companion was approximately in the position $a$ in the figure. For many years it remained visible through telescopes of high quality as it traced the part of its orbit remote from Sirius, but in the year 1890 as it passed $b$ it disappeared to the finest instruments in the world as its light was lost in the brilliant rays of the star. From these it has now emerged and is again visible at $c$.

By repeatedly measuring the position of the companion with respect to Sirius we have obtained its relative orbit. If, however, we record the positions of both star and companion with reference to fixed stars in the same telescopic field, we may obtain the actual orbit of each orbit. This has been done, with the result shown in fig. 4. It appears from this that the distance of Sirius from. the common focus $O$ of the ellipses is at every instant one half that of the companion, from which we arrive at the conclusion, assumed in our previous study of the figure, that the mass of Sirius is double that of the companion. The result is remarkable in view of the fact that the star gives more than 30,000 times the light of the companion.

The period of mutual revolution of two attracting masses increases with their distance apart, or, more accurately,

1 The apparent orbit as actually traced from observation is not that of fig. 3 . In the case of a body describing an elliptical orbit round a centre of attraction as focus, the actual form of the orbit as seen from a distance will only be preserved if it is presented squarely to the line of vision. If oblique, the orbit is seen in perspective. It still, however, appears elliptical, but the degree of ellipticity is changed. Happily, however, the attracting star no longer appears in the focus, and this fact enables us to tell whether the orbit is tilted, while the displacement of the star from the focus of the perspective ellipse enables us to determine the extent of tilt and to allow for it. The figures illustrating the lecture are those of the actual orbits, the distortion due to tilt having been corrected. 
with the major axis of the relative ellipse, though not in simple proportion. This law is illustrated in the increasing lengths of the periods of revolution of the planets of the solar system with their increasing distances from the Sun. The period also depends upon the masses of the stars. From these considerations we should expect that, though irregularities would appear due to different systems of double stars possessing different masses, upon the whole, the closer the components of a double star, the

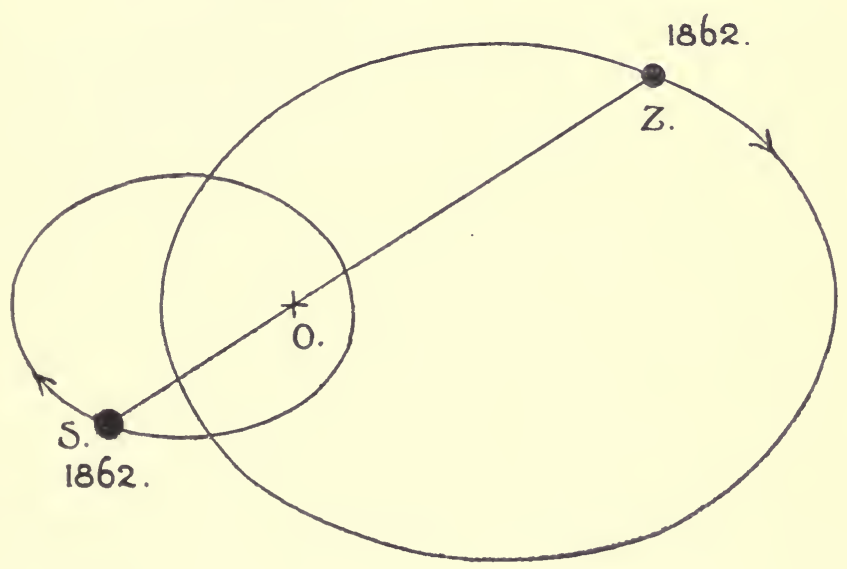

FIG. 4. Orbits of Sirius and companion.

more rapid would be its revolution, and this is actually found to be the case. The first binary systems discovered, being naturally those in which the components are widely separated, had long periods of revolution, and in many instances no trace of relative motion has so far become apparent, though other considerations leave little doubt that the systems concerned are true binaries. With increasing telescopic power, double stars with closer and closer components have been recognized, and with these discoveries correspondingly shorter periods of revolution 
have appeared. With one possible exception the shortest period so far recognized among double stars whose components are separately visible in the telescope is that of $\delta$ Equuilei, which is completed in 5.7 years. From this evidence there can be no doubt that if it were possible to effect further refinement in the construction of telescopes still closer binaries with shorter periods would be discovered. We shall presently find very complete evidence of the existence of binary systems, consisting of pairs of stars so close as to lie far beyond the possibility of direct observation.

Before proceeding, however, it will be of interest to inquire how we are to interpret the phenomenon of a double star.

When, as a consequence of the establishment of the Copernican system of astronomy, it was clearly recognized that the stars were suns, it was confidently believed that each was, like the Sun, the centre of a planetary system of its own, giving life to a system of revolving worlds. It is difficult, however, to find a place for a double star in such a scheme. It seems impossible for a planet to accompany a pair of suns, and at the same time to follow so regular an orbit as to experience the slightly varying conditions of temperature that are necessary to maintain life. While but few double stars were known it was possible to regard them as celestial monstrosities, objects out of accord with the harmonious universe in which they found a place, but in view of the increasing numbers that have been discovered, their exceptional character can no longer be maintained. A recent high authority has gone so far as to regard a double star as the normal condition of stellar existence, and single stars, like the Sun, as exceptions.

How then have double stars come into existence? A 
view that is at first naturally suggested sees in them two stars, originally independent, that, happening to approach each other, have been since kept together by their mutual attraction. In such a case, however, the stars would gain as the result of their attraction during their approach sufficient velocities to carry them back to their original positions, however remote these were, a law illustrated in the rush of comets through the solar system. Further, the chance of two stars isolated in space being drawn together at all seems far too small to account for the existence of so large a proportion of doubles, while the same process would lead to a rich distribution of triple, quadruple, and more complicated systems, and all these are extremely rare. We are therefore led to inquire whether the components of a double star may not have been physically related from the first, and to endeavour to imagine some process by which, in accordance with known physical laws, they may have been developed from a parent mass.

In 1755, the German philosopher Immanuel Kant, descending for a time from the sublime heights of the Critical Philosophy, imagined a scheme by which the Solar System might have been developed by the operation of natural law upon a vast cosmic cloud. Forty-one years later, a speculation of essentially the same character was suggested by Laplace, and, owing to his higher authority as a mathematician and as an astronomer, it has since been generally associated with his name. According to Laplace, the matter now composing the Sun and planets existed at one time as an intensely heated mist, which extended beyond the present limits of the system. This nebula would have been spherical, but for the fact that it possessed a motion of rotation round a diameter, and this, by the action of centrifugal force, caused it to become spheroidal, flattened along the axis of 
rotation and extended round the Equator. As the nebula parted with its heat by radiation into space, it contracted, and so, by the operation of a well-known mechanical law, the motion of all its parts became accelerated. The increased rate of rotation developed a greater centrifugal force, and this, in its turn, caused a further emphasis of the spheroidal form. After a time a stage was reached at which centrifugal force, always greatest at the Equator, had there acquired a value at which it balanced the gravitation of the mass. Consequently, upon further shrinkage, the equatorial parts were left behind in the form of a ring, still retaining the motion of rotation that they possessed while forming a part of the parent mass. By the repetition of the process a number of revolving rings were shed in succession. The central nucleus of the shrunken nebula now forms the Sun, and the rings, breaking and condensing, have formed the planets and their satellites. To account for the small mass of the whole planetary system in comparison with the Sun, and also for the very moderate speed of rotation of the Sun itself, Laplace found it necessary to assume that the nebula was very strongly condensed towards its centre. It was regarded, indeed, as little more than a tenuous atmosphere of the Sun pervading the space now occupied by the planetary system.

The Nebular Hypothesis has always been regarded, as it was regarded by Laplace himself, as a speculation rather than as a scientific theory. Whether the nebula would develop in the manner imagined, whether it would shed rings, and whether, if they were shed, they would condense into planetary globes, are problems that might conceivably be matters of mechanical investigation, but they have so far proved to bebeyond the powers of modern mathematics. The fact, however, that the process does appear to be, in its essential character, a probable one, while its results 
are in such admirable harmony with the leading features of the Solar System, has caused it to be generally regarded as representing, at any rate approximately, the manner in which the planets came into existence.

The phenomena of double stars suggest, however, that another mode of development is possible from a nebula, and in its attack upon this problem it seems possible that mathematical science has been more successful. It is true that the solution of the problem is still far from complete, and that it cannot be regarded as having passed beyond the limits of speculation. It rests, however, upon two independent researches, that, whatever application they may have to double stars, are in themselves of very great interest. One of these, starting from a study of the mutual relations of the movements of the Earth and Moon, is due to Professor George Darwin; the other, which is a masterly study of the changes experienced by certain rotating masses of fluid, has been developed by M. Poincaré. We shall first sketch very briefly the leading points of Darwin's investigation of the past history of the Moon.

From the fact that the intensity of the Moon's attraction is greater upon the parts of the Earth that are nearer to it than upon the parts that are more remote, there arises a tendency for the Earth to become stretched along the diameter that is at any particular instant directed towards the Moon ${ }^{1}$. If the Earth were fluid, it would yield to this tendency, but, as it is in the main solid, it is unable to do so. The waters upon its surface are however free, and they consequently flow, continually tending to accumulate in two high tides, one immediately under

1 The stretching tendency is accentuated by another cause, the convergence of the attraction exercised upon different parts of the Earth by the Moon towards the Moon's centre. 
the Moon, and the other at the part of the Earth's surface that is most remote from it. If the period of the Earth's rotation was the same as that of the Moon's revolution round it, the Moon would continually face the same regions of the Earth, and in the course of time, possibly in a few months or years, the water would reach a position of equilibrium, forming permanent high tides at the opposite ends of the diameter that would then be permanently directed to the Moon.

This simple condition is, however, profoundly modified by the Earth's rotation. As the Earth turns under the Moon in a period of slightly less than twenty-five hours, the regions presented to the Moon-those at which the water tends to accumulate-are continually changing, and before any portion of water could move appreciably towards them, the forces acting upon it would change and it would be urged in some other direction. The problem thus becomes extremely complicated. The general result, however, is, that in its continual endeavour to move towards the ends of the terrestrial diameter that is at each given instant pointing to the Moon, the water on the Earth's surface is thrown into the continual motion that we recognize as tidal ebb and flow.

If the movement of water were unresisted by friction, tidal ebb and flow would possess no cosmical significance, but friction is experienced in the motion of the tidal wave over the surfaces of shores and estuaries, and in internal motions of water itself. The destruction of motion by friction develops heat, and the Earth is consequently warmed by its tides; moreover, since heat is a form of energy, some other form of energy, equivalent in amount, must disappear in producing it. From considerations of a not very difficult nature, it can be shown that this energy is that of the Earth's rotation, so that we are 
presented with the remarkable fact that the speed of the Earth's rotation is being reduced in consequence of the tides. The period of the Earth's rotation determines the day, and consequently the day must be increasing in length. No doubt the rate of increase is now very slight, but there can be little doubt that this has not always been the case. The Earth was at one time a mass of molten rock, in which bodily tides must have been formed, while friction itself must have been far greater in the case of such a viscous mass than in water. Further, as we shall see, the Moon must have been nearer the Earth than it is now, and its tide-producing power consequently more intense. Under these conditions we can well imagine that the loss of rotation proceeded at a comparatively rapid pace, and that the day was formerly far shorter than it is at the present time.

The slackening of the Earth's rotation is not, however, the only result of tidal friction. A reaction upon the Moon is inevitable, and it appears that, as a necessary consequence, the Moon must recede from the Earth, its orbital speed decreasing at the same time. Its period of revolution round the Earth, which we may define as the month, is therefore increasing, so that in consequence of the tides, the day and the month are both becoming longer. It follows, however, from simple considerations that this cannot continue indefinitely. The day is increasing, and so also is the month, but there must come a time when the day must increase more rapidly than the month ${ }^{1}$, and it must ultimately overtake it. The length of each will then be fifty-five of our present days. The Earth, then rotating in the same period as that of the Moon's revolution round it, will continually present the

1 This time has already been passed in the case of the Earth-Moon system. 
same regions to the Moon, as the Moon already presents the same face to it. At the ends of the terrestrial diameter that will then be constantly pointing towards the Moon, permanent high tides will accumulate; ebb and flow, and with it tidal friction will cease, and a state of stable equilibrium will be reached ${ }^{1}$. It is impossible to determine the epoch of this stage, but under the most favourable conditions it must be measured by hundreds of millions of years from the present time.

There can be little doubt that we have in these actions an explanation of the fact that the Moon continually presents the same face to the Earth. The mass of the Earth, which determines its attractive power, is eighty times that of the Moon. Its tide-generating power is therefore eighty times as great. There is no water upon the Moon, so that tidal friction seems to have been sufficient to bring its periods of rotation and revolution into coincidence while it was still in a molten condition.

It is interesting to follow the future of the Earth-Moon system ; but it is of greater interest to trace the past. In the past the Earth must have rotated more rapidly, the Moon must have been nearer, and it must have revolved in a shorter period than at present. From the application of mathematics to the problem, Darwin has shown that there must have been a time when the Moon was quite close to the surface of the Earth, and, when in this condition, the further suggestive fact appears that its period of revolution, the month, coincided, as it will again coincide in the last stage, with that of the Earth's rotation, the day. Both must then have been between

1 The argument assumes that the terrestrial oceans will remain fluid. If owing to decrease in solar radiation the oceans should become frozen, tidal friction will cease before the final condition described above is reached, and, with it, further development of the process. 
three and five hours in length. In this first, as in the last condition, we see the Earth and Moon rotating as a whole about their common centre of mass, each continually presenting the same face to the other. While, however, in the first condition they are nearly in contact and represent a passing phase, in the last they are far apart and their condition is permanent ${ }^{1}$. It is possible to show that the first stage could not have occurred less than $50,000,000$ years ago.

By the application of the laws of mechanics to the phenomena of the tides, it is thus possible to trace the history of the Moon backward to a time when it revolved in close proximity to the Earth's surface. At this point the investigation indicates the beginning of tidal friction between the two bodies, and beyond it the mathematical expressions have no physical meaning. Speculation, however, cannot rest satisfied without an attempt to imagine a still earlier condition of the system, and there is an obvious direction for it to take. It is certain that, when rotating in a period of between three and five hours, the Earth could not have been far removed from a condition of instability, roughly resembling a grindstone on the point of breaking into fragments through the violence of its rotation. It is an obvious suggestion that the Moon is a part of the Earth detached from its parent by the rapidity of its rotation, and there can be little doubt that it actually came into existence in this way.

It is probable that, at the time of their proximity and their probable separation, the Earth and the Moon were both in a liquid, or even in a partially gaseous, condition.

1 In this study of the Earth-Moon system, a lesser tidal effect caused by the Sun has been ignored. The consideration of the solar tidal effect would slightly affect the forms of some of the above statements, but would not affect the general conclusions, so far as they are applicable to the study of this lecture. 
It is interesting to notice that under these conditions neither could have been spherical. Their rapid rotation would of itself cause each to tend to a spheroidal form, while a further modification would result from the tendency of each to become elongated by tidal action along the diameter directed to the other. Darwin has determined the forms assumed by two equal masses of fluid of uniform density throughout when rotating as a whole and almost in contact, and a section of the pair through their common axis of rotation is given in fig. 5 .

Axis of rotation.

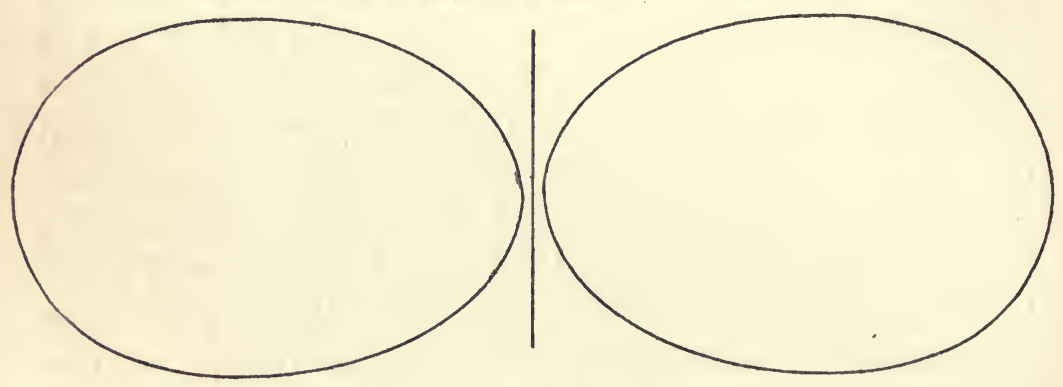

FIG. 5.

The forms of the bodies are not described by any simple geometrical figure.

At a time in the remote past, the Earth and the Moon were almost in contact and they were then revolving round their centre of mass precisely as if they were connected together by a rigid bar. From this it seems an almost unavoidable inference that they were formed by the division of a single mass, and that this mass was either fluid or gaseous. It may appear to those who are uninitiated in the mysteries of the science of hydrodynamics that it should be a simple matter to decide whether or not such a division of a rotating fluid mass is in accordance 
with the known laws of mechanics, and, if it is in accordance with these laws, to trace the manner in which it may be effected. Unfortunately, however, the difficulties presented by the problem are so great that it has so far defied the power of modern mathematics. Recently, however, certain remarkable investigations have been made upon the behaviour of rotating masses of gravitating fluid, which, while not supplying an actual solution of the problem, do undoubtedly appear to throw some light upon it. The results of these investigations appear to me to be so interesting, and so suggestive in their astronomical application, that I propose to consider them in some detail.

Let us imagine a mass of fluid of any form to exist in celestial space, and to be so far removed from other bodies as not to be appreciably affected by their gravitational attraction. Let us further assume that the fluid is incompressible, and that it is of uniform density throughout. By the law of gravitation each portion of it will attract every other portion with a force that is inversely proportional to the square of the distance between them. Under the influence of these attractions between its parts the mass will tend to acquire the form of a sphere. If, at the instant at which our attention is first directed to it, every part is at rest, the mass will in fact ultimately assume a spherical form, in which all its parts are at rest. The sphere is therefore regarded as a figure of equilibrium for the gravitating fluid. Further, if the spherical mass is subjected to any accidental disturbance, if for instance, it were drawn out of form by the tidal action of a passing planet, it would, when the disturbing influence has ceased, reassume the spherical form. For this reason the figure is regarded as one of stable equilibrium.

Next, let us suppose that a motion of rotation is imparted 
to the sphere. By the action of centrifugal force, every part exerts an outward pull on the mass, a pull directed from the axis of rotation. Yielding to these pulls, the figure ceases to be spherical and becomes a spheroid-the form of an orange-flattened along its axis of rotation. This is the actual form of the Earth. The spheroid is therefore a figure of equilibrium of the rotating mass, and, if the speed of rotation is not excessive, it is a figure of stable equilibrium, for it again tends to reassume its form after being disturbed. As the speed of rotation is increased, the centrifugal forces increase, and a further flattening is the result, but for a time the stable spheroidal form is retained. The section through the equator remains a circle, and every section through the axis of rotation is an ellipse of the same form. The laws regulating the spheroidal form of a mass of rotating fluid were investigated by Maclaurin about the year I 750.

We have supposed the liquid to be initially at rest, and have assumed that a rotation is impressed upon it after it has acquired a spherical form, the sphere passing in consequence of the rotation into the spheroid. If, however, the different portions of the liquid are not initially at rest, the mass may, under simple conditions, though it need not under more complicated ones, assume the form of the rotating spheroid directly, and it is more natural to regard the spheroid as having been formed in this way. In the time of Laplace the spheroid was the only figure of equilibrium known for a mass of rotating fluid, and the spheroidal form was naturally adopted by him as the initial state of the nebula of his hypothesis. Since then, however, other possible forms of equilibrium have been discovered, and these are connected with Maclaurin's spheroid in a very interesting manner.

We have left the spheroidal nebula rotating with 
moderate speed. Let us now imagine a steady twist ${ }^{1}$ to be applied to it. It will rotate faster and faster, and it will in consequence become more and more flattened. It is, however, now known that this process will not continue indefinitely. A stage will be reached at which the spheroid will have the greatest flattening that is practic-

Axis of rotation.

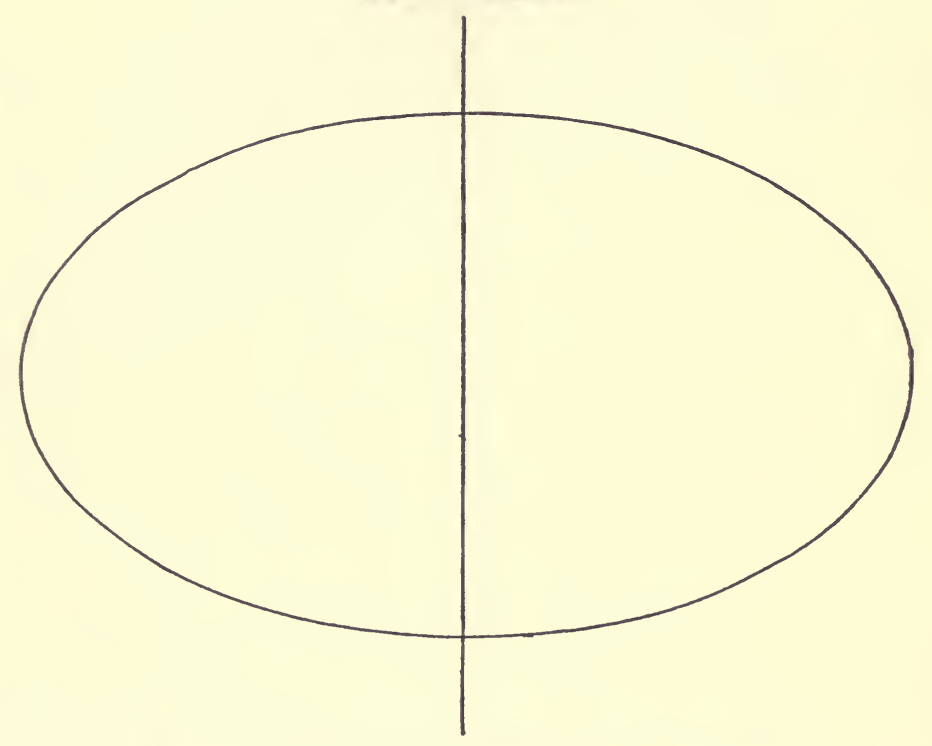

FIG. 6. Critical form of Maclaurin's spheroid.

ally possible. Its euqatorial diameter is now $\mathrm{I} \cdot 7$ times its polar axis, and its form is shown in fig 6 . Upon continuing the twist, the form of the mass ceases to be spheroidal. It is true that spheroids with a greater flattening are conceivable as figures of equilibrium, but beyond this point they are in an unstable condition. Like

1 The term 'twist', in its meaning as adopted in scientific language, is not used accurately here, but it will convey a more definite impression to the general reader than the more scientifically accurate expression. 
a needle balanced upon its point on a plate of glass, they would, upon the slightest disturbance, instead of tending to revert to their original condition, tend to diverge still further from it, and, this action continuing, the whole mass would rapidly break up in some unpredictable manner, unless it happened to fall into some other form that was stable. Outside mathematical analysis permanent unstable forms of equilibrium are impossible.

On passing this first critical stage in its history, the mass of rotating liquid ceases to be a spheroid. It acquires Axis of rotation.

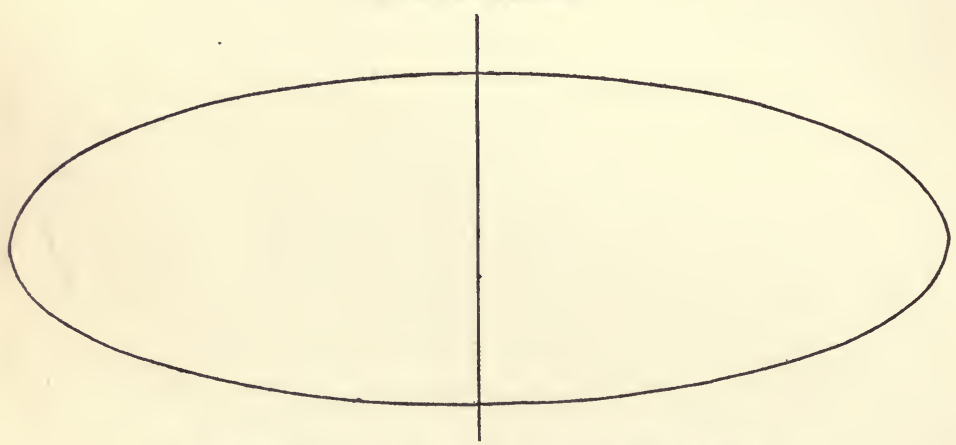

Fig. 7. Critical form of Jacobi's ellipsoid.

a figure in which the equatorial section is no longer a circle. This section becomes, like the one through the polar axis, an ellipse. The figure is now known as an ellipsoid, and it was found to be a figure of equilibrium of a rotating liquid mass by Jacobi about the year I 850 . Upon continued application of the twist the ellipsoidal form becomes more and more developed, the polar axis becoming shorter, the greater equatorial diameter increasing still further, and the one at right angles to it becoming less. The remarkable fact, however, appears, that with continued application of twist the period of rotation, instead of becoming shorter, as had previously been the 
case, now becomes increasingly greater. For a time the condition of the ellipsoidal form of the liquid mass is stable, and all continues well, but at length it reaches a second crisis in its career, beyond which, if it were to continue as an ellipsoid, it would become unstable and would meet with inevitable destruction. The section through its polar axis and its greatest equatorial diameter is now represented in fig. 7. Denoting its polar axis by I, the greatest equatorial diameter is $2 \cdot 9$, and the least equatorial diameter, drawn through the centre and at right angles to the paper, is $\mathrm{I} \cdot 25$. Its period of rotation is now $I \frac{1}{3}$ times that of the spheroid in fig. 6 .

A third form of stable equilibrium which the rotating fluid mass may assume after passing the limiting form of stability of Jacobi's ellipsoid, has been recently discovered by Poincaré. It has somewhat the form of a pear. Upon one side of the axis of rotation of Jacobi's ellipsoid, the longer equatorial axis becomes shorter, and on this side the figure becomes fatter, while upon the other side the long equatorial diameter becomes still longer, and upon that side the figure becomes thinner. There is, further, a suggestion of constriction or waist round the figure not far from its middle. M. Poincaré's first attempt to trace the form is shown in fig. 8 , but Darwin has recently shown that the resemblance to a pear is not so strongly marked as Poincaré had supposed. This point marks the limit to which it has so far been possible to apply mathematical methods to the problem, and the further development of the pear-shaped figure is unknown. Although, however, we can only proceed by speculation, it is impossible to restrain the imagination in its attempt to trace the course of development further. Darwin and Poincaré are both of opinion that the resemblance to a pear would become more pronounced by the further development of the waist, 
and that ultimately the mass would divide into two parts, the parts rotating as a whole, and necessarily round the original axis passing through the centre of mass of the two. If this should be so, we should be presented with a condition closely analogous to the one that Darwin has shown to have been that of the Earth and Moon at an early stage of their history.

In tracing the development of the fluid mass, we have

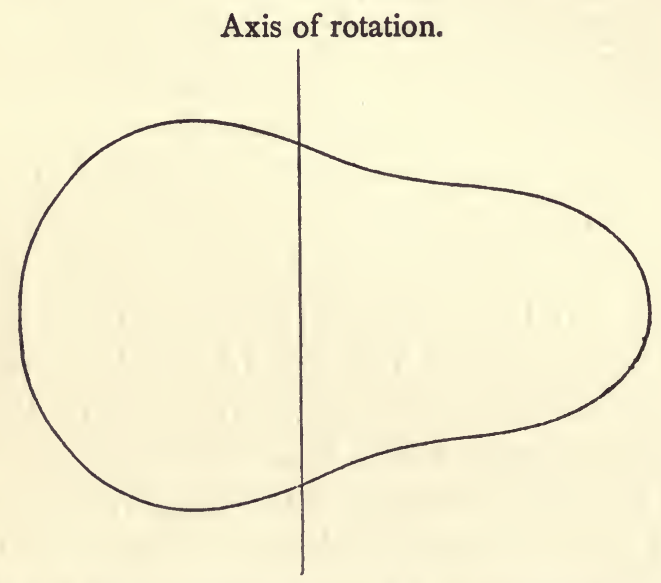

FIG. 8. Poincaré's pear-shaped figure of equilibrium.

supposed its various changes to have been caused by a continuously applied twist, which would, if the mass had been rigid and therefore incapable of undergoing change of form, have produced a continual increase in its rate of rotation. If, however, we assume an initial rotation, and if we suppose the fluid to shrink, as it would if it were gaseous through the radiation of its heat into space, the same series of changes would occur without the application of impressed force. Left to itself the spheroid would shrink, and its rotation would in consequence be accelerated. The polar flattening would therefore increase, and 
this would continue until the limiting form of Maclaurin's spheroid was reached. Beyond this, upon further contraction, the form of Jacobi's ellipsoid would be assumed and the period of rotation would increase. The ellipsoid would become longer in proportion to its girth, and on passing the critical condition of fig. 7 , would be replaced by the pear. In the development of the pear mathematical methods fail, but there can be little doubt that its form becomes more pronounced, and that a waist develops that ultimately divides the mass into two bodies, nearly in contact, and in rapid revolution. At this point exact methods resume their sway. Each of the separate masses produces tides in the other, their axial revolution is checked, and the distance between them increases. From this point their history is that of the Earth and Moon.

The reader will be inclined to ask whether this scheme may not replace that of Laplace, and whether the planets may not have been developed from the Sun, and subsequently thrown off to their present distances by tidal action, as the Moon appears to have been developed and thrown outward from the Earth. There are, however, fatal objections to this rather attractive view. By imagining their tidal effects undone, the Moon and the Earth have been traced back to a condition when they must have been very nearly in contact. A similar operation performed upon the Sun and Earth reduces the Earth's distance from the Sun by quite an insignificant amount. The Earth must have been formed not far from the present position of its orbit. A similar objection appears in any attempt to explain the origin of the satellites of any other planet in this way. The system of the Earth and Moon is unique in the solar system. In no other case does a satellite possess nearly so great a mass in proportion to that of its primary, and it is owing to 
this high proportion that so great a separation has been possible as the result of tidal friction.

The Earth-Moon scheme of development does not therefore replace that of Laplace. Neither does it, so far as is yet known, exclude it. Laplace imagined a nebula strongly condensed towards the centre; Poincaré one of uniform density throughout. It by no means follows that the developments of such different bodies will proceed along similar lines, but the relation between them may conceivably be a problem to be successfully attacked in the future.

There is, however, another problem to which we may reasonably hope to apply the Earth-Moon method of development with greater success. It is to systems of double stars. In all cases in which comparison is possible, the components of a double star do not differ greatly in mass, and therefore a wide separation by tidal friction is conceivable. If, in any double star, the rates of rotation and revolution of the components were known, and also the dimensions of the system, Darwin's method would enable us to trace it back to the first stage at which tidal friction became an operative factor, and, if the components should then appear to be in close proximity, their origin would not be doubtful ; but, unfortunately, it seems impossible to expect that such complete data will ever be at our disposal. There is, however, another way in which we may hope to test the theory. If it furnishes a true expression of the development of a double star, it is reasonable to expect that its different stages will be represented in existing systems, that there are, in addition to the cases revealed by the telescope, in which the separation is necessarily great, and in which accordingly we are tempted to see systems in an advanced stage of their life-histories, others in which the separation is small, and 
others again in which the components are nearly in contact. The theory will be further strengthened if in such cases there appears other and independent evidence of an early stage of stellar life. We have now to see how such systems have been discovered, and by what methods their dimensions have been made a matter of scientific investigation.

The first evidence of the existence of very close doubles was suggested by the behaviour of a certain class of variable stars. That a certain number of the stars vary in brightness from time to time has been known from very early times, and their number has been enormously increased by recent observations. In some cases, the variation is of an extremely irregular nature, the behaviour of the star in the past supplying no indication of the future course of its light changes. In the majority of cases, however, the variation displays at least an approximation to a definite periodicity, and, in a few instances, the light rises and falls in a definite period, and in a manner that is repeated with almost mathematical exactness. Of these regular variables, the oldest known, as well as the easiest to observe, is situated in the constellation of Perseus, and is known as Algol $^{1}$ or $\beta$ Persei.

Algol appears normally as one of the very brilliant stars, its brightness being slightly below that of the second magnitude. After shining steadily for about fifty-nine hours, however, its light begins to fade, and in the course of four-and-a-half hours it has descended to thirty-eight per cent. of its original brilliancy. It then, and apparently without a pause, begins a recovery which is completely effected in a slightly longer time than that

1 The name Algol is derived from the Arabic Al-Ghoul, the demon; it was probably so named from its changing appearance. 
of its decline, after which it shines steadily with its original light for another period of fifty-nine hours, when the variation is repeated, and so on indefinitely. The position of Algol in Perseus, near the brilliant constellation of Cassiopeiea, may be found from any star map, and its variation in brightness can be readily followed by comparing it from time to time with other stars in its neighbourhood.

The mathematical regularity of the variation led Goodricke in 1783 to suggest that it was caused by the revolution of a dark companion round the star. The orbit of the companion was assumed to lie in a plane that made at most a very small angle with the line of sight, so that at every revolution the star was partially eclipsed. The theory obviously met the main facts of the case, and it was very much strengthened by an interesting investigation by E. C. Pickering in 1888 . From a great number of observations made by Schönfield between 1862 and 1870 , Pickering deduces the law according to which the loss and the recovery of the star's light was effected. Such a law is conveniently represented by a curve constructed upon the principle of the ordinary barometer chart. Along a horizontal line equal distances are set off to represent equal intervals of time, and from each point verticals are drawn, each representing by its length the brightness of the star at the instant indicated by the point from which the vertical is drawn. A smooth curve is then drawn through the tops of the verticals, and this is the light curve of the star. The actual light curve of Algol is given in fig. 9.

Having deduced the law of variation from Schönfield's observations, Pickering proceeded to determine from geometry the law according to which the light change should take place, upon the assumption that it was caused 
by the transit of a dark sphere across the face of a bright one. The result of this investigation, which, although it involves laborious calculation, presents no real difficulty, was that, upon assuming certain relative proportions for the star and companion and their distance apart, as well as for the inclination of the orbit to the line of sight, a theoretical light curve was obtained that practically

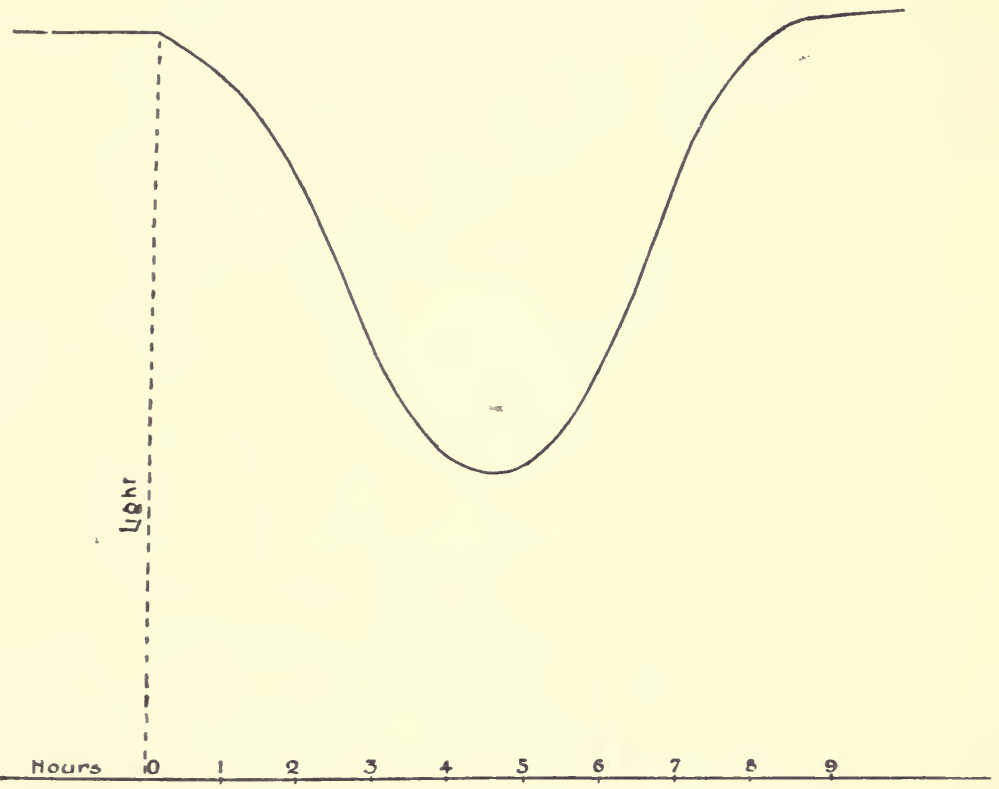

Fig. 9. Light curve of Algol.

coincided with the one deduced from observation. The actual assumptions necessary for this agreement were that, taking the radius of Algol as unity, that of the companion is represented by 0.764 and the distance between their centres is 4.33 . Further the plane of the orbit makes an angle of $3^{\circ}$ with the line of sight. The system is represented to scale in fig. 10, and the view of it as it 


\section{THE EVOLUTION OF}

would appear from the Earth in fig. II. It will be noticed from fig. I I, that at the central eclipse the companion is just, and only just, projected upon the disk of the star ${ }^{1}$.

Pickering's investigation had left little doubt of the truth of the eclipse theory of Algol's variation, but evidence of a still more definite nature was soon to be

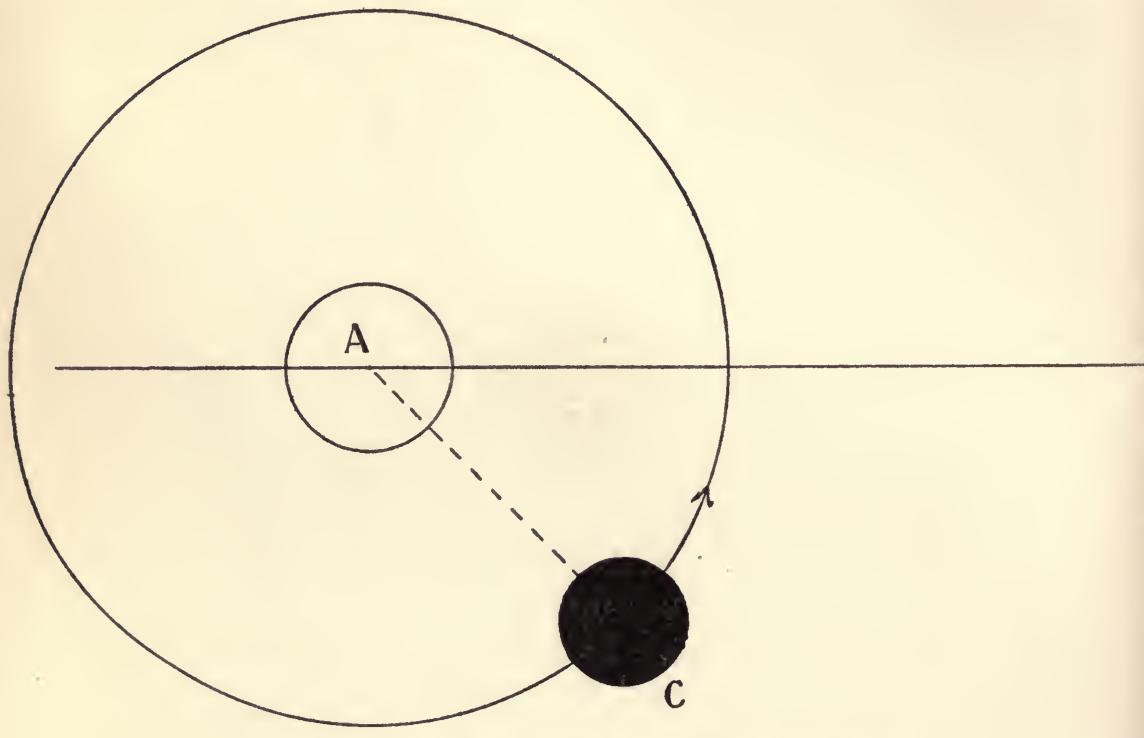

FIG. 10. Algol and relative orbit of companion.

forthcoming. If Algol is accompanied by a revolving companion, it must, as we have seen, be itself in revolution, star and companion describing similar orbits round their common centre of mass. In fig. Io, the relative orbit of the companion is represented, and Pickering had

1 The reader will scarcely need to be reminded that it would be impossible to recognize the disk of the companion upon that of the star by the telescope. The distances of the stars are so great that all of them appear as mere specks of light through the finest telescopes. 
shown that it must be, at any rate approximately, circular. There is no indication of the relative masses, and therefore it is not possible to determine the position of the centre of mass upon the line $A C$, but the general nature of the motion is represented in fig. 12 , in which the mass of the star $A$ is assumed to be twice that of the companion $C$, so that, if $O$ is the centre of mass, $O C$ is twice $O A$. If then we assume that the solar system lies in the direction $O E$, the eclipse will begin when $A$ and $C$ approach the line $E^{\prime} O E$ from opposite sides, and it will be deepest when their centres lie upon it. Hence according

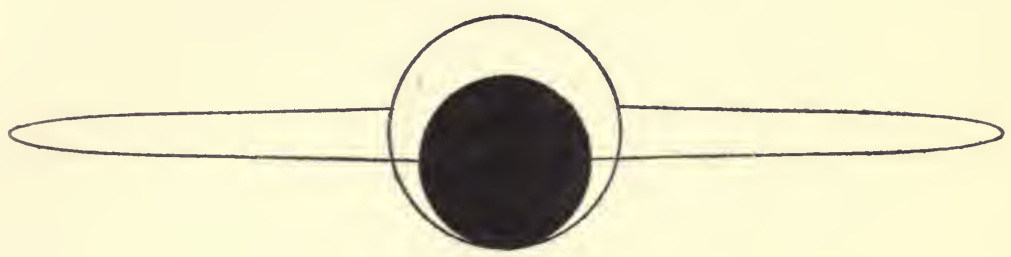

Fig. I I. System of Algol as presented to the Earth.

to the eclipse theory Algol must be in motion, and it becomes a question whether its motion may not be detected. If a telescope of sufficient power were possible the star would appear to swing up and down, from $K^{\prime}$ to $K$ and back, in a period coincident with that of the light variation, but the detection of this is quite impossible. The star should, however, possess another motion relative to the observer. It should, after eclipse, be approaching him, as it traversed the half of its orbit, $P Q R$, while for the half-period before an eclipse it should be receding along the half $R S P$, the rates of approach and recession reaching their greatest values at $Q$ and $S$ respectively. These movements have actually been detected, and their s. 2. 
detection and measurement form one of the most striking chapters in the history of astronomy.

It is well known that the apparent pitch of a note is affected by the approach or recession of the body emitting it, and the effect is capable of simple explanation. A body emitting a musical note does so by generating a regular succession of waves in the air, which travel outwards from it in every direction. These waves enter the ear, and the sensation of pitch that is developed depends solely upon the rapidity with which the individual waves

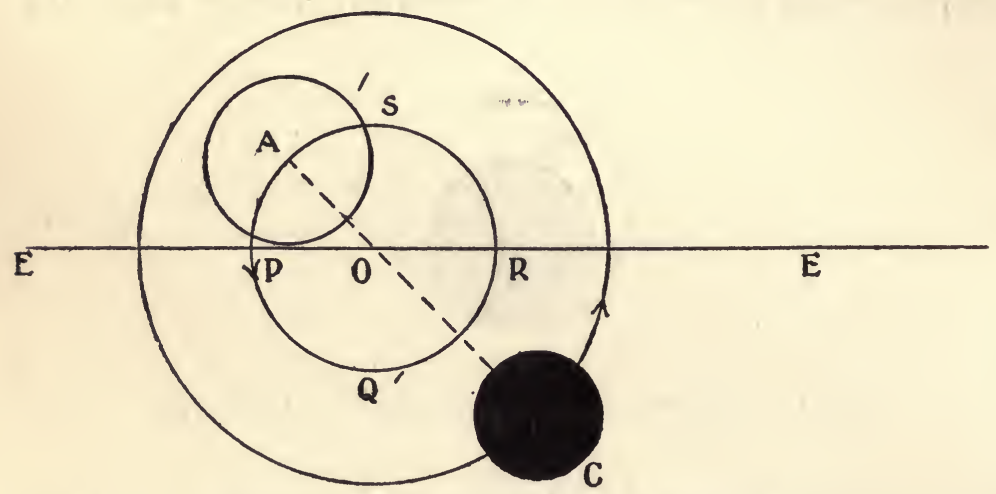

Frg. 12. Orbits of Algol and Companion.

follow one another. This again clearly depends upon their length, the shorter waves, following in more rapid succession than longer ones, giving rise to the sensation of a higher pitch. Now, if the sounding body were to approach the observer, it would, by its motion, shorten the waves in front of it that it had already generated, by crowding them together, and the pitch should consequently be raised. Similarly, by a motion of recession, the pitch should be lowered, by the waves in the rear of the moving source of sound being drawn out and lengthened. These effects are fully con- 
firmed by observation, and are readily noticed in the sudden and very appreciable fall in pitch of the whistle of an engine as it passes an observer at express speed.

Light is also due to wave motion, being the result of waves generated in, and transmitted by, the ether of space; colour, or, more definitely, position in the spectral series, being determined, like the pitch of a note, by the rapidity with which individual waves follow one another. Owing to the great speed of light, i. e. that of the ether waves, no change of colour that could be appreciated by the eye could be produced by any velocity with which we are acquainted, but the position of a ray in the photographed spectrum of the brighter stars can be measured with such accuracy that the effect of an approach or recession at the rate of a mile in a second can now be detected. The spectrum of a star consists, like the spectrum of the Sun, of a band of light displaying the successive colours of the rainbow, but crossed by a number of dark lines indicating the absorption of definite rays by its atmosphere. In the case of an approaching star these lines should consequently be raised in pitch, or displaced towards the violet end of the spectrum. By a motion of recession they should be lowered towards the red. Such displacements are now recognized in the spectra of a great number of stars, and their study forms an important branch of astronomical observation. From it, it is possible to deduce the component of a star's velocity in the direction of the line of sight.

In 1889 Vogel applied this principle to the investigation of the problem of Algol. The spectrum of the star was photographed at a quarter of its complete period before its minimum, when, according to the eclipse theory, it should be at $S$, receding from the Earth with 
its greatest speed, and again at a quarter of the period after its minimum, when it should be at $Q$, and approaching with greatest speed. As was anticipated, it was found that in the first photograph the spectral lines were displaced towards the red, while in the second they were displaced towards the violet. Further, the amount of displacements indicated an orbital speed for the star of twenty-six miles per second. It follows from a simple calculation that, to travel with this speed and to occupy 68.8 hours in describing its orbit, the radius of the orbit -the distance $O A$ in fig. I 2-must be approximately a million miles.

In many other stars phenomena are presented very similar to those of Algol, and almost all of these have been discowered during the last quarter of a century. In some cases, as in that of Spica, a regular oscillation of the spectral lines indicates an orbital motion of the star, and consequently necessitates the presence of a companion, but the motion is unaccompanied by any change of light, so that the orbits must be so inclined to the line of vision that eclipses are avoided. In several instances a variation of light takes place, as with Algol, but the stars that exhibit it are too faint to furnish spectra capable of sufficiently exact measurement to establish the fact of motion by their means. In a third class of stars, illustrated by Mizar, the dark lines of the spectra regularly open out into doubles and close again with a concertina-like motion. There can be no hesitation in interpreting this effect as due to a double star, of which both components are luminous. When the line joining them is at right angles to the line of sight, one of the components is approaching and the other is receding from the Earth, so that the lines of their separate spectra, becoming displaced in opposite directions, are separately 
visible. At a quarter period later, recession and approach cease, as the components travel directly across the line of sight, and, their spectra being superposed, the result appears single. In the greater number of these cases the period of a complete oscillation of the spectral lines, and therefore that of mutual revolution of the component stars, is measured by a few days. In the case of Spica it is four, and in that of Mizar, twenty days.

From the variations of their light, and through the movements of the dark lines in their spectra, we have thus become acquainted with double stars, the components of which are so close as to evade all possibility of telescopic separation. In many of these cases the actual distance separating the stars must be very small : in the case of Algol and its companion for instance, it is, as we have seen, less than five times the radius of the bright star. We shall conclude our study by considering the case of a star that has received a great deal of attention, and which presents features that have not even yet received an entirely satisfactory explanation.

In 1784 Goodricke observed that the light of the bright star $\beta$ Lyrae varied between fairly wide limits. The remarkable character of its variation has attracted a great deal of attention since, and in no other instance has the law of light variation been determined with so high a degree of accuracy. Between I840 and I 860 Argelander made more than 1,500 comparisons of the star with others in its neighbourhood, and from them expressed the law of light variation by means of a curve. This curve is reproduced in fig. $13^{1}$.

Referring to the figure, it will be seen that the variation is essentially different from that of Algol. Instead

${ }^{1}$ The variation may be easily followed by the naked eye, by comparing the star with others in its neighbourhood from time to time. 
of a long period of constant light, the variation is continuous, and instead of one minimum, there are two, but one of them is more marked than the other. The complete cycle of changes is effected in 12.9 days, and it may be conveniently regarded as divided into four stages, which are of almost equal duration, each lasting for rather

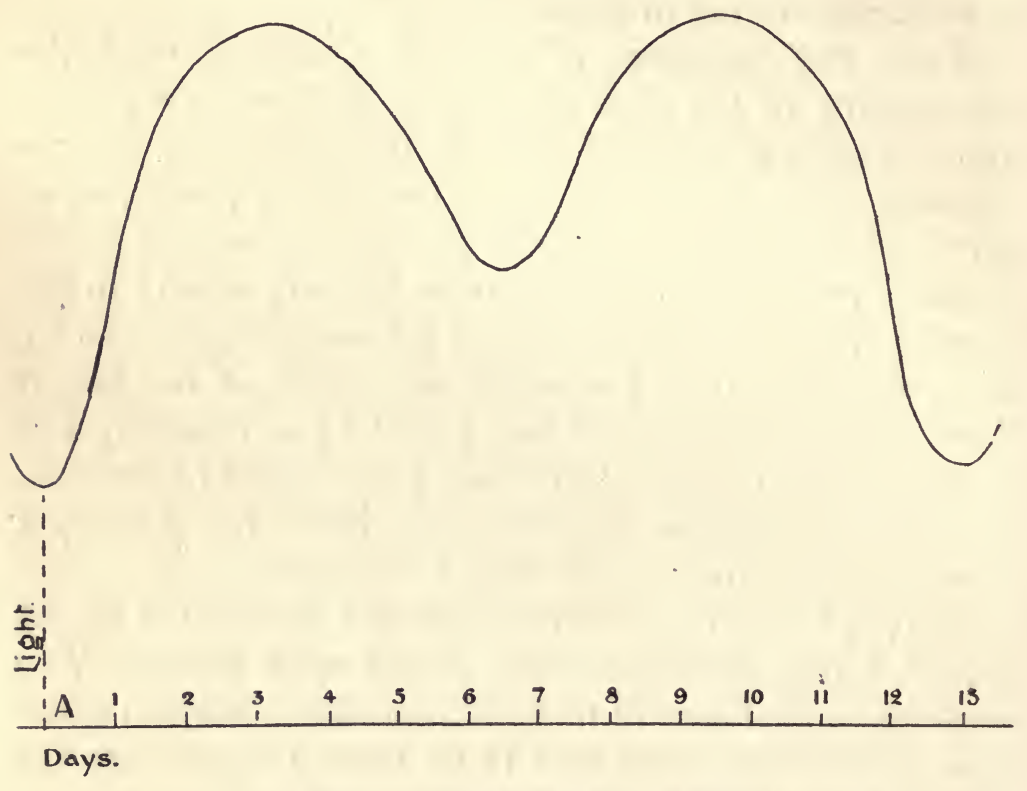

Fig. 13. Light Curve of $\beta$ Lyrae.

more than three days. Starting from the instant $A$, in the figure, the time of the chief minimum, the brightness of the star is 34 per cent. of its maximum. In the first stage it rises rapidly to its maximum value of Ioo. It then at once begins to decline, and in the second stage falls to a secondary minimum with a brightness of 64 . In the third stage it rises again to 100, and in the last it descends to its chief minimum of 34 . The cycle is then 
repeated indefinitely with almost mathematical regularity, though there is distinct evidence that the period has increased since the first observations were made.

A little consideration will show that it is possible to explain the variation of $\beta$ Lyrae by an eclipse theory, so far as its main features are concerned. The brightness at the chief minimum is almost exactly $\frac{1}{3}$, and that at the secondary minimum almost exactly $\frac{2}{3}$ of the maximum, and we will, for the purpose of illustration, assume that these simple values are exact. Now imagine two stars $A$ and $B$ to revolve in circular orbits round their common centre of mass, or more simply, and this comes to the same thing as far as the light changes are concerned, imagine $B$ to revolve round $A, A$ remaining fixed. Suppose further that the plane of revolution is presented edgeways to the eye, and that the stars are of equal size, but that $A$ is twice as bright as $B$. Let us call the light of $A, 2$, and that of $B, 1$. At a particular instant in its revolution $B$ will pass between $A$ and the observer, and will completely eclipse it. The light received is now $\mathrm{I}$, that of $B$ alone. This corresponds to the chief minimum. In a quarter of the period of revolution $B$ will have moved a quarter of the way round $A$, the observer will receive light from both stars, and its amount will be 3 , that of $A$ and $B$ together. This is the maximum. In the next quarter period $B$ will move behind $A$, and will be entirely concealed. The light, now that of $A$ alone, is 2. This is the secondary minimum. By the end of the next quarter, $A$ and $B$ will again be clear, the light rising to 3 , and at the end of the fourth quarter the original condition will be restored.

It is possible to explain two equal maxima alternating with two unequal minima by the hypothesis of central eclipses in every conceivable case, though it is 
generally necessary to assume that, in addition to their differing in brightness, the stars are unequal in size. It appears that it is only, as happened to be the case with the approximate values that we assumed, when the sum of the two minima is equal to the maximum, that the stars must be of the same size. To account for the actual minima of $\beta$ Lyrae which are 34 and 64 per cent. respectively of the maximum light, it is, in fact, necessary to regard the surface of $B$ as $\frac{17}{18}$ of that of $A$, and its surface brightness - the light radiated by each unit of area of its surface-as $\frac{6}{11}$ of that of $A$. The reader will find it an interesting exercise to deduce the two minima values upon these assumptions.

So far we have found a perfect explanation of the maxima and minima of stars of the $\beta$ Lyrae type, and we may now proceed a stage further. According to the eclipse theory, the light should acquire its maximum value as soon as the rise of $B$ clears that of $A$ in the course of its revolution round it, and it should remain at this value until $B$ in its turn begins to be concealed behind $A$. It is clear that the greater the distance between the stars, the longer the time for which this maximum phase should last. In the actual case, however, there is no constant maximum at all, the light begins to fade immediately after reaching its maximum, and $B$ must therefore begin to be eclipsed by $A$ immediately it has finished eclipsing it. The only condition under which this can happen is when $B$ is in actual contact with $A$, gliding round close to its surface, so that we seem to be presented with a case similar to that of the Earth and Moon in the first stage of their separate existence, a conclusion that is very suggestive.

Having reduced the system of $\beta$ Lyrae to that of two stars in contact and in mutual revolution, we can now 
apply a further test, suggested by the form of the light curve. It is not a difficult matter to determine by geometry what this form should be, under the assumptions of the relative sizes and brightnesses of the stars that we have been compelled to make, and it is actually represented by the continuous curve in fig. I4. It will

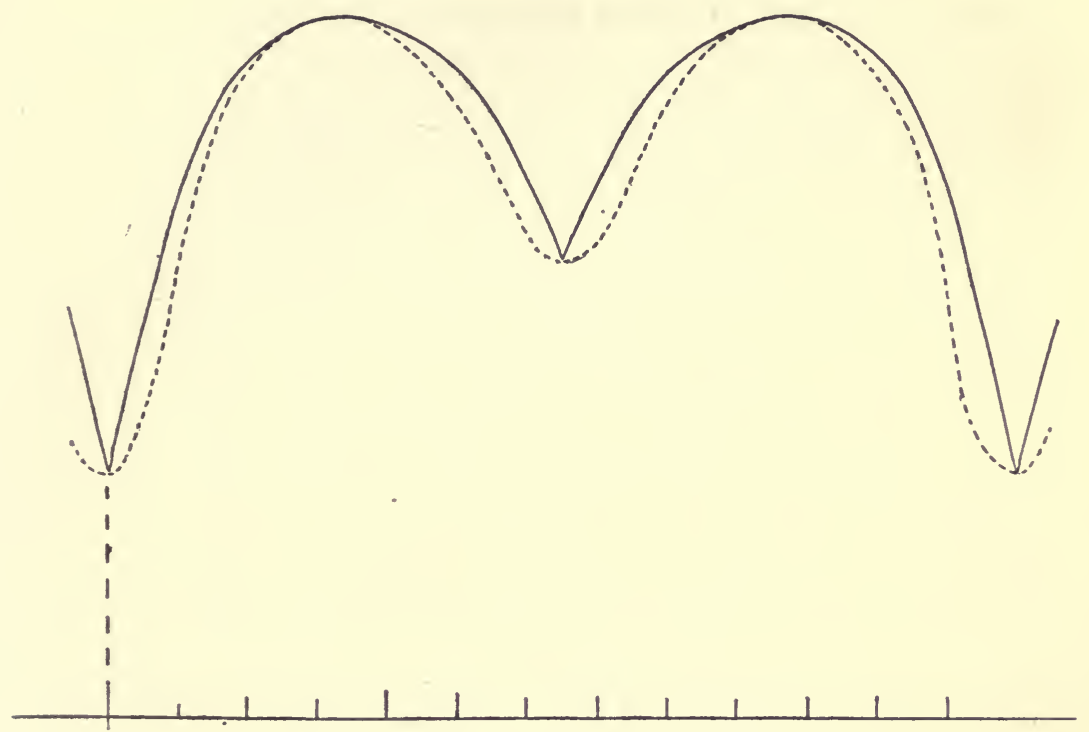

Fig. 14. Light Curves of $\beta$ Lyrae.

Calculated

Observed

be seen at once that this is very far from corresponding with the observed curve, which is introduced in the same figure for the sake of comparison and is represented by the dotted curve. The observed maxima are more, and the observed minima are less, sharply defined by observation than they are predicted by the theory, so far as we have developed it up to the present. 
We have, however, retained an assumption that can hardly be maintained if the component stars are actually very near each other. The stars have been supposed to retain their spherical forms. We have, however, seen that, when close together, each star must elongate the other by tidal action, the elongation being in the direction of the line joining their centres, and that forms will be assumed similar to those represented in fig. 5. Acting

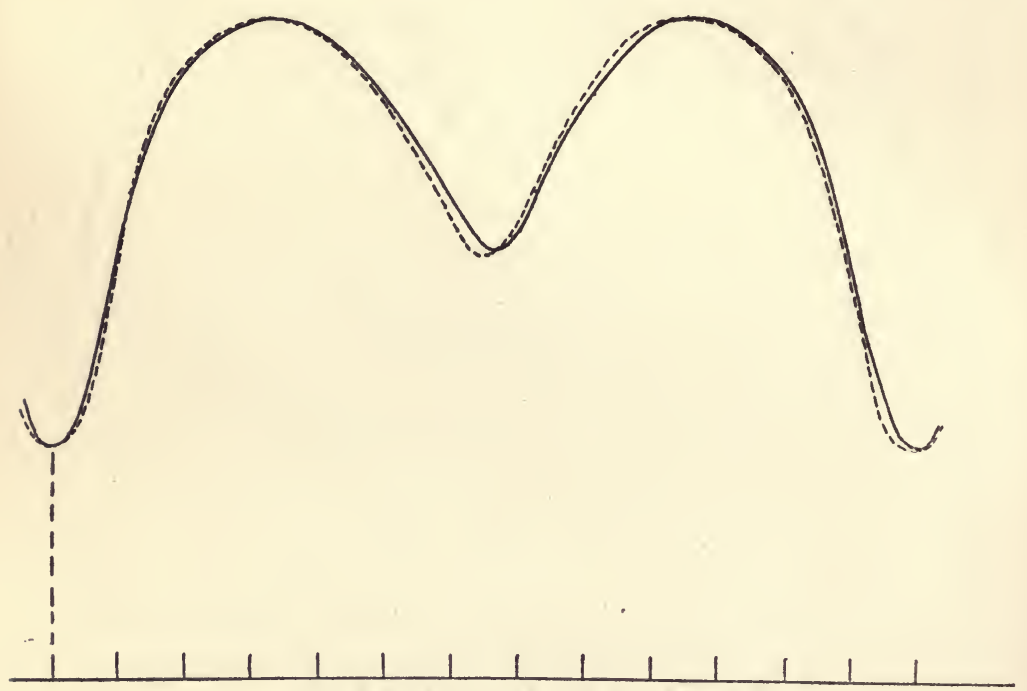

FIG. 1 5. Light Curves of $\beta$ Lyrae (Myers).

Calculated curve Observed curve

upon this suggestion, Myers attacked the problem of the light curve in 1898 , upon the assumption that the stars were prolate (or lemon-shaped) ellipsoids, each with its longest axis directed to the other. These forms are indeed not precisely those indicated by the tidal theory, but they are probably a sufficiently close approximation 
to them. The result of the investigation was very remarkable. Myers' theoretical curve is given in fig. I5, in which it is directly compared with the observed curve, which is again reproduced in the dotted line. The coincidence is so nearly exact as to leave little doubt that the system imagined actually represents that of the star. The data determined by Myers as giving the best agreement with observations were as follows :

I. The stars are similar prolate ellipsoids, the shortest diameter of each being $\frac{7}{9}$ of its greatest.

2. The ellipsoids differ in size, their greatest diameters being in the ratio of 8 to 9 .

3. The larger body is $\frac{4}{10}$ as bright as the smaller.

4. The distance between their centres is $\frac{15}{16}$ the greatest diameter of the larger star.

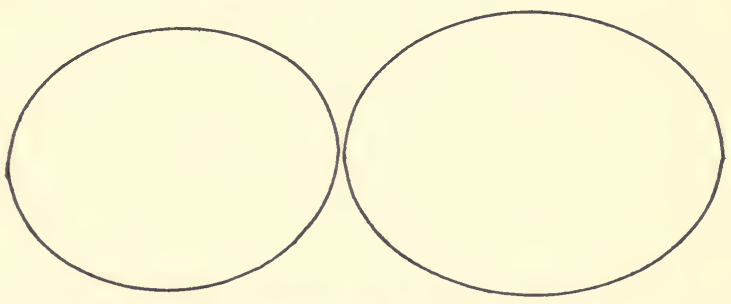

FIG. I6. System of $\beta$ Lyrae (Myers).

The relative dimensions of the system are represented in fig. 16.

We are tempted, therefore, to regard $\beta$ Lyrae as representing the earliest stage of separate existence of the components of a double star. There is also independent evidence to suggest that $\beta$ Lyrae as well as variables of the Algol class in general have densities that are very much less than that of the Sun, and this in itself suggests an early stage of development. Further, in the case of $\beta$ Lyrae, the spectrum is so closely allied to that of 
a nebula, that it appears probable, from that fact alone, that the star is not far removed from the nebulous condition. These conclusions clearly support the view of development that we have adopted, but it is unfortunately impossible to enter here into the details of the attractive branches of astronomical research upon which they are based.

The very considerable eccentricities of the orbits of telescopic double stars appear at first sight to be a difficulty in connexion with the theory, but, although the problem of eccentricity has not been completely worked out, it seems possible that it may actually tend to confirm it. Darwin has shown that a gradually increasing eccentricity of orbit is itself a necessary result of tidal friction, and that it will be most marked as the components approximate to each other in mass. Whether, however, this is sufficient to account for the high eccentricities actually observed is a matter upon which it is at present impossible to express a definite judgement. It is, however, a suggestive fact, that, upon the whole, high eccentricities are characteristic of the telescopic doubles in which separation is great. In $\beta$ Lyrae, and in stars of the Algol class, the details of light variation demand orbits that differ but slightly from the circular form.

Whether or not a double star is actually formed by the division of a rotating nebula in the manner suggested by our study, it is certain that tidal friction must play a part, and possibly an important one, in the separation of the components, and in the introduction of eccentricity into their orbits; and there appears little doubt that the ellipsoids of Maclaurin and Jacobi, as well as the pearshaped figure of Poincaré, play important parts in the general scheme of stellar evolution. 


\section{VII}

\section{ANTHROPOLOGY}

THE EVOLUTION OF CURRENCY AND COINAGE

(Sir Richard C. Temple, Bart.)

THE task set before me to-day is to elucidate for students the vast Natural Science of Anthropology by the selection of typical data from one small branch of it and by generalizations drawn from the study of these. I may add that practically in a single lecture it is possible to consider but a portion of this small branch, and even then I feel constrained to apologize for the rapidity of my survey.

The terms Currency and Coinage are frequently used in common parlance as denominating the same thing, though this is not the fact. The latter term is really included in the former. All coinage is used for currency, but currency does not by any means consist only of coinage. Coinage is, however, so late a development of currency that a consideration of it offers a suitable subject for the present purpose-a study of the working of the mind of man in devising means for the convenience of mankind. It goes further, indeed, and illustrates, perhaps better than any other subject, the methods by which the human mind meets the daily requirements of mankind. 
The actual use to which currency and coinage are put is to enable human beings to exchange commodities or conveniences with each other. The object of this lecture is to examine, so far as the time available permits, how far human beings manage to put currency and coinage to their use.

The idea of exchange, in the sense of a gift for an equivalent in return, is itself a development. I doubt if it is present in young children, and I throw out the suggestion that it would be an interesting occupation for those in a position to do so, to determine the average age at which the child proceeds definitely from the idea of the gift to the idea of barter, or gift for gift. [Since delivering this lecture I have been told by a competent observer of very young children in numbers that the idea of gift for gift, without knowledge of relative value, comes to the highly civilized English child with the taught knowledge of counting, and that 'swopping' of articles of assumed equal value, involving the idea of relative value, comes much later with the taught knowledge of games based on counting. It will be presently seen that the progression of ideas here is precisely that which has been observed amongst primitive savages.]

At any rate there are in existence savages, who have not even yet developed beyond the initial stage and have no definite notion of barter. The most primitive savages of my acquaintance-I have learnt too much caution to say the most primitive in existence-are the Andaman islanders in the Bay of Bengal. Among them, excepting in the absolute essentials of life and in the most cherished articles of food, all possessions are communal and freely given away, even children, for the asking. Among many of them also, who are still out 
of contact with civilization, the utmost development of the idea of barter is the making of a gift in expectation of another gift in due course in return. If the return is neglected there is a row. This you will perceive is, however, not real barter. It has not got so far. It is not even the giving of a definite thing for another definite thing in return. That idea involves a further development, which some of these savages in their untutored state, and only some, possess in that fluid fashion in which they possess all ideas.

This development has been brought about by environment. Those of them that have chanced, owing to local conditions, to possess the monopoly of a thing have exchanged it, in an indefinite manner, for something else they could not readily get. That is, they gave cookingpots made of a special clay, found only in certain parts of the islands, to friendly tribes for gifts made immediately in return.

Now I ask you to observe, that even in such primitive conditions as these, the human mind has been working on regular lines of thought development. First there is the gift, then simply a gift for another gift. Then there is the gift of an article that a particular class only possesses for a gift in return of an article that it wants. In all this there is as yet no idea of relative value. It is not yet true barter. That requires a still further development. But in the very little distance we have proceeded on our journey we have stumbled across a general law of nature, which will meet us everywhere on our further progress and in every anthropological investigation-the pressure of contact on human development. As it is the possession by some of the Andamanese of a thing that their neighbours cannot 
procure for themselves that has made them commence to barter.

Of the next step in development, that of bartering something for another thing held to be of equal value, we have in books of travel very many instances. The ordinary method adopted by primitive savages of dealing with strangers, in obedience to a general anthropological law of suspicion of strangers which we cannot discuss now, is to lay out their merchandise on a given spot and watch the stranger from a safe distance lay out his exchanges alongside. When the stranger has departed to a safe distance they come out of their hiding-place and examine the exchanges, and either accept or reject them, or go back and wait for more. As a typical instance I may quote from an old book of travel among the Greenlanders in 1635, which tells us that 'their manner of trucking is thus : they put all they have to sell together, and having picked out among the commodities that are brought to them what they like best, they put them also together and suffer those they deal with to add or diminish till such time as they are content with the bargain.' This goes a little further than the savage habit just explained, for the Greenlanders were not apparently afraid to meet the stranger face to face and truck with him.

We now come to the instructive subject of trucking with savages by barter, about which many wrong statements have been made in error, to the detriment of the European trader, owing to want of anthropological knowledge and inquiry. This is in reality a question of exchange value looked at from the point of view of the buyer and seller, and you will perceive later on that even in the most primitive and most highly civilized bargains the principle is the same, and that the human 
mind argues in the same fashion under both conditions. The blame is usually cast on the trader, who is charged with buying valuable articles from the savage for a mere song. This is fair neither to the trader nor to the mental capacity of the savage.

There is in the Mergui Archipelago in the Bay of Bengal a wild Malay tribe known as the Selungs, miserably poor savages from our point of view. They are exploited by Chinamen from British territory, who barter with them 'a little cloth, paddy, tobacco, and perhaps the smell of opium now and then,' for 'black coral, eagle wood, and so on,' which these people gather. The value of the goods given by the Chinamen is to them about a fifth of what they get. The goods obtained by the Selungs for what they give are to them of vital importance, and 'they are enabled thereby to maintain a wild independence.' There is nothing unfair in the bargain. The Chinamen have to take what they have received elsewhere before they can make a profit, and the savage has given something that is of no value to him for something which is very great value. This is what is meant by the term 'trade,' or bargaining for mutual benefit. We have in fact reached now the point of trade by barter.

As there have been so many animadversions wrongly cast on European traders as to this matter, let us go a little further into it. In $187 \mathrm{I}-2$ an expedition had to be undertaken against the Lushais, savages on the eastern borders of Assam in India. Of some of these savages it was reported that 'they brought down large quantities of india-rubber, which they eagerly exchanged for salt, equal weights, and as the value of the rubber was more than four times that of the salt, any individuals who could command a large supply of the latter had an 
excellent opportunity for a little profitable business.' This was the civilized man's profit. Now let us look at the bargain from the savages' point of view. We can do so because it has been recorded also in another Report, which tells us: 'In former times these tribes made all the salt they required for their own consumption from salt springs, and they say that to make enough salt for the requirements of an ordinary family a man's labour was required for three months. A man can now collect sufficient india-rubber in one month to exchange with Bengalee traders for more than enough salt to last him and his family for a year. So that a man who chooses to occupy himself three months in collecting rubber will, by bartering the same for salt, have a large surplus of that article, with which to trade with the Southern Tribes, who, they say, are willing to give one maund of rubber for a quarter maund of salt.' Here is a clear instance of a trade by barter, which is of profit to both sides, each from its own point of view, owing to the differing conditions in which they respectively lived and acted.

You will, of course, understand that what we call money has in the stage of trade by barter no value whatever as such. A specimen of it in such circumstances is merely a commodity, which may have no value, or have a value very different from that which it possesses for us. This fact is of importance in the present inquiry, as it shows that the idea of money is so much a later development than the idea of barter, that in the stage of barter it exists not at all. In proving this from instances, I am able to illustrate further the working of trade by barter as above explained. Of the Greenlanders already noticed it was said that 'there is no money in the countrey, being so happy as not to know 
the value of Gold and Silver. Iron and Steel they most esteem, and prefer a Sword or a Hatchet before a Golden Cup, a Nail before a Crown piece, and a pair of Cisers, or a Knife before a Jacobus.' And during the Lushai expedition above mentioned, a rupee, which is roughly a florin, having been given for a fowl on one occasion the savages would only thereafter exchange fowls for rupees, though the rupees could be got back again for the base metal coin of a neighbouring semi-civilized state. In 1893 among the Shan tribes of the Chinese border rupees could not buy a pony, though small silver coins of the same number, and of course of much less value to us, could. Amongst other Shans, copper coins alone could purchase anything, any kind of silver failing to be attractive, and there being no difference in the value placed upon a rupee and its eighth part.

The reason in each of the above cases is the same and clear. The savages in question had either no use for money at all, or they had a use respectively for the base metal, the small silver and the copper, but none for the rupee, which to us was of very greatly the highest value. The adherence of the Lushais to a rupee as the exchange equivalent of a fowl was due to an accident. Having got into their heads by a chance that to us a rupee was the proper exchange for a fowl, they stuck to it from an unreasoning suspicion that, unless they did so, we were in some unascertainable way cheating them; and their subsequent exchange of the rupees so acquired for what was to us base metal rubbish was from their point of view to their advantage.

In the course of this small further progress on the road leading to money, we have stumbled upon another general anthropological law, which is true alike of these 
primitive dealings with savages and of the most highly developed transactions of the European Exchanges; viz., that trade will accommodate itself to any circumstances and evolve a modus vivendi between any two apparently irreconcilable parties, showing that in similar conditions human reasoning will produce a similar result. The proposition may be generally stated thus: the capacity for reasoning remains the same, whether the society in which a human being finds himself is primitive or highly advanced.

There is another circumstance in the cases quoted, which shows the constant nature of human reasoning. The context of the passages quoted proves that wherever a trade has been arrived at, there is a great eagerness to trade. Indeed, it is a common observation among travellers that savages, who have arrived at the stage of trading, are inveterate bargainers. It does not require a knowledge of money to turn a man into a keen trader, and even savages will acquire not only 'tricks of the trade,' but will carry them to a great state of perfection. Instances innumerable could be cited, from among those to whom only barter is known as the means of trade, of a deep insight into their own interests and of a capacity to take advantage of current conditions to their own profit. The strongest instance that has come under my observation, covering the whole point, is from the Hottentots of South Africa. John Jourdain, who was at the Cape in 1608 , says they bought from the natives 'a cowe for a peece of an ould iron hoope of a yard longe, and a sheepe for halfe soe much. And many tymes, havinge sould them to us, yf we looked not the better to them, they would steale them agayne from us and bringe them agayne to sell; which we were fayne with patience to buy agayne of them, without givinge. 
any foule language, for fear least they would bringe us noe more.' Here we have the whole story of smartness in trade among those who could only barter. We have the price of iron, which they could not manufacture, in terms of the live stock which they possessed in great abundance. We have also the taking advantage of the chance position of the sellers of iron to get as much of that coveted commodity as possible for as little in the way of live stock as possible. In our modern parlance, they sent in their bills twice to those who could not or would not resist that nefarious proceeding. I put the matter thus to show the identity of reasoning in the unprincipled savage and in the unprincipled civilized trader.

There peeps out in this instance also a fact, which indicates a great advance in the reasoning that originally led to trade by barter. It will have been seen that roughly the Hottentot put twice the value on his cows that he did on his sheep. That is, his sheep and his cows had for him a relative value, more or less fixed. This is really an all-important advance in reasoning, as it is not until the idea of relative value is reached that the idea of currency can arise.

I will carry this just a step further by an instance within my own experience of trade by barter. I once had to acquire for the Government about eight-anda-half acres of coco-nut covered land in the island of Car Nicobar in the Bay of Bengal. I first carefully and literally walked the boundaries, fixing them approximately with a prismatic compass to the great awe of the sellers, and then gave them without hesitation what they considered as much as they could dare to ask, viz., twelve suits of black cloth, one piece of red cloth, six bags of rice, twenty packets of China tobacco, twelve 
bottles of commissariat rum. The bargain as a money transaction was very advantageous to the Government, but I found out afterwards why the people had so readily accepted it as being advantageous to them, by working out the value of the articles bartered in terms of their currency, although I am quite sure they had no notion that they possessed such a thing, and could never have explained what the term meant.

In commencing, however, an explanation of the rise of the idea of currency and of the sense of the term, let me quote some dealings with the less civilized peoples inhabiting the British province of Burma and its neighbourhood. As everywhere else, the Government of India has to preserve order among the uncivilized by means of fines and some sort of pecuniary penalties and enforced compensations, and it has to collect revenue in some shape or other, and for these purposes it must perforce have some means of apportioning values. But the people only understand barter, and the notion of relative value is entirely rudimentary. In these circumstances in Assam, among the Kacharis, the British Courts have drawn up for their own use a regular scale of fines and revenue in terms of the domesticated animals kept by the people; e.g. a man's revenue would be assessed, not at Rs. Io, but at a big buffalo; a fine would be fixed not at a quarter of a rupee, but at a cock and two small hens. So amongst the Chins in Burma, tribute by way of a customary present would not be Rs. Io, but a full-grown hog, and a fine or compensation for injury would not be Rs. 5, but a silk jacket. Even the old native Government of Burma had to adopt a system akin to this, for at the time of the first Burma war of 1824 it levied fines, as a variant of the very ancient notion of slavery for debt and partly as a kind 
of blood-money, on the value of the human body on the following scale in terms of British money:-
A new-born male child .
Rs. IO
A new-born female child
A boy .
25
A girl.
I $7 \frac{1}{2}$
A young man
75
A young woman . . . . . $83 \frac{1}{2}$

The necessities of civilized Governments obliged them thus to set up, and educate populations up to, the idea of relative values. By making the people accustomed to giving domestic animals and articles a fixed relative value, the idea of currency became possible to them, as we shall presently see. In fact, we are now present at the very birth of that product of human reasoning which is called Currency. For the term Currency means the use of an article commonly possessed as the medium of exchanging actual property between buyer and seller. Currency is thus the means of measuring the relative value of other articles.

The step in evolution now under consideration is so important for the present argument that I pause to make clear the difference between the conditions when trade is carried on by means of barter only and when it is carried on by means of a currency. Barter, as we have seen, is the exchange of possessions - of grain, say, for fruit, of an adze for a knife. But when our daily transactions become so far complicated as to require some other article in common domestic use to be interposed between the grain and the fruit and between the adze and the knife, i.e. a medium of exchange between the articles bartered, we have set up a currency and a medium of exchange. To make my meaning 
clear, let us put ourselves into the position of a tribe of tropical savages. Thus: You and I and the rest of our tribe have all got coco-nuts in varying quantities and can find a use for them every day. I want fruit and you want grain, but instead of exchanging my grain for your fruit I give you six pairs of coco-nuts for the fruit I want, and later on you come to me and give me five pairs of coco-nuts for the corn you want. In the same way I give you my adze for coco-nuts and you give me your knife for coco-nuts. Here we are bartering through a medium and coco-nuts are our currency.

Currency consists in fact of articles real or imaginary used for account, which is a highly civilized way of saying 'for measuring the relative values of different articles of use'-so many coco-nuts make one adze: so many coco-nuts make one knife, to follow the fashion of the tables of childhood. This is a universal and inevitable condition not confined to wild peoples, and shows once more the constant nature of human reasoning.

An essential condition for an article to become currency is that it should be commonly required or possessed. Obviously if an article is to become a popular medium of exchange, everybody must possess it in some degree or other. Possession of it in a small degree we term 'poverty' : its possession in a high degree we term 'wealth.' I will explain this point among uncivilized peoples from circumstances within my own experience. In the Nicobar Islands from all time, and we have knowledge of the Nicobars from the seventh century, coco-nuts have been and still are the currency of the people. They and the trees on which they grow are the staple products of the country, and the most valuable possession of the inhabitants. They play a great part in finding them in food and drink, and in materials for 
housing, clothing, and furniture. Thus they are in constant and daily use, and they are employed for currency, i.e. for measuring the value of other articles and as the medium in exchanging them.

I can give a strong instance of this from what I have myself seen. On April 5, 1896, when I was in charge of the islands, the people of Mus in Car Nicobar had occasion to buy a large racing-canoe from the people of Chowra Island. They proceeded to value the canoe at 55.000 coco-nuts, but they are a lazy people and had no intention of fetching such a large quantity down from the trees in their possession. So they paid for the canoe in a great number of articles, each valued in coco-nuts, nearly all of which were in their possession as the result of trading in coco-nuts with such foreigners as Burmans, Chinese, Malays, and natives of India. The list of these articles is interesting and goes to prove my point, but I have no time to give it you now. It included domestic animals, utensils and implements, cloth, beads, silver articles, and even British money. This transaction induced me to set the local Government agent to try and ascertain the approximate value in coco-nuts of such trade articles as the Nicobarese require for domestic and other uses, and his inquiries produced a long list, from needles at twelve coco-nuts a dozen and matches at twenty-four coco-nuts per dozen boxes to Turkey red cloth at 1,600 coco-nuts the piece. Now in this list a two-anna bit, which is the eighth of a rupee, was valued at sixteen coco-nuts, and the rupee itself at 100 : but it will be perceived that eight times sixteen is I28. The reason for the discrepancy is that the little piece of silver is used for one sort of ornament and the big piece for another sort, and their value in coco-nuts to these people depends on their value as ornaments, 
and not on their relative weight combined with fineness, or, as we should say, their intrinsic value. I need hardly say that the Nicobarese do not recognize coins as a medium of exchange.

There is in addition a mass of evidence from all parts of the world showing how savages and some semicivilized peoples employ natural articles as currency. Thus rice has been used up till quite recent times as currency in daily transactions in many outlying places about Burma and the neighbourhood: and in some parts of China this is the case no doubt still, and it was so in Kashmir in the sixteenth century. Cloves were currency in the Moluccas at the same time, and fish in some parts of the Malay Archipelago, at any rate in 1820 . Salt is another article that has been used, as noticed by even the earliest travellers, in China, Burma, and the hills all round, and in many parts of India. $U p$ to the time of the first Burmese war in 1824 , at any rate, cotton was the currency between Arakan and Burma. Of live stock I need hardly say much, as the use of these for measuring values is a very widely spread instance to the point. But chickens were currency in the Maldives off the south-west coast of India in the fourteenth century, and pigs in Tibet and oxen in Central Asia in much later days. The Lushais of the Assam-Burma borders reckon in buffaloes, and among the Khonds of Eastern India, the people of the Meriah human sacrifices; in 1845 'the value of all property was estimated by the Maliah Khonds in "lives," a measure which requires some adjustment every time it is applied; a buffalo, a goat, a pig, or a fowl, a bag of grain, or a set of brass pots, being each, with anything that may be agreed upon, a "life." A hundred lives on an average may be taken to consist of ro bullocks, Io buffaloes, IO sacks 
of corn, Io sets of brass pots, 20 sheep, Io pigs, and 30 fowls.'

The next point to observe is that currency in natural articles has not by any means been confined to savages or semi-civilized communities. In Iceland in 1420 , stockfish or dried cod was the currency, and in the early British colonies all through the seventeenth century, there is much and most interesting evidence to show that the currency covered a great variety of articles: tobacco, corn, wampum, sugar, rum, cotton-wool, mahogany, molasses, ginger, indigo, skins, and so on. In 1643 , in Massachusetts, wampum strings were made a legal tender, and tobacco was rated under penalties at $3^{s}$. per lb. in Virginia in 1618. So sugar, tobacco, and other things were made into monetary standards in the West Indies in the seventeenth and eighteenth centuries. Dried cod-fish was a circulating medium in Newfoundland till much later. Even as late as I 708, tobacco, to quote an old Report, was 'the Meat, Drink, and Cloathing and Money' of Maryland; and of tobacco, as a currency, there is a good story told about Virginia in I620-2I. In that year ' 150 young and uncorrupt' girls were imported as wives for the colonists, and were rated originally at $100 \mathrm{lb}$. of tobacco or $£^{\mathrm{I}} 5$, but subsequently at the increased rate of $150 \mathrm{lb}$. of tobacco or $£^{22}$ Ios. And we are told 'that it would have done a man's heart good to see the gallant young Virginians hastening to the waterside when a vessel arrived from London, each carrying a bundle of the best tobacco under his arm and taking back with him a beautiful and virtuous young wife.'

In Barbadoes the colonists commenced with a currency chiefly in cotton and tobacco, but also in indigo and 'fustic-wood.' About I640 sugar became the currency 
and was legal tender from 1667 onwards, coined money being established in I715. In the Leeward Islands, books and accounts were kept in terms of sugar, and even as late as 1740 it was officially stated that 'the value which is put on sugar, rum, cotton, and other commodities, the growth of the Leeward Islands, is called currency there.' The variety of the 'other commodities' was considerable from time to time; tobacco, cotton-wool, indigo, ginger, molasses, and so on; and their rating was fixed by the Government, just as we saw the Indian and Burman officials rating live stock and so on for the wild Lushais and Chins. This went on more or less till $\mathrm{I} 784$. In British Honduras mahogany in the form of logwood lasted till I 785 , one of the most unwieldy currencies yet invented, except perhaps the 'millstone' money of the Caroline Islands in the South Pacific. In the Bermudas, which was the first of the colonies to start a coined currency, tobacco was the currency until 1658. All this is exactly paralleled by the elk teeth which passed for twenty-five cents among the Shoshone and Bannock Indians in the United States, and the unus or plaited fibre armlet of Borneo, which passed as three cents of the trade dollar per bundle of fifteen armlets.

It is the collation of facts such as these that proves the existence of the anthropological law of the constancy of human reasoning, which I have so often already pointed out. In similar conditions the mind of civilized and uncivilized man works in much the same directions and produces much the same results. It is a law that must in all anthropological investigation be ever remembered. The mind of man, like the other parts of him, is always the same mind, though variously developed in individuals and groups of individuals. Before pro- 
ceeding further I will give a strong additional instance of this. Commercial dealings when the currency is in kind are strictly analogous to those when it is in money. Even among such wild people as the Chins of Burma there is a weight in and a weight out, i.e. the dealer takes goods into his store at a different weight to that which he uses in weighing the same goods out of it. The difference in the weights represents his profits. In Siam and all over the land of the Shans, where civilization is higher than among the Chins, two recognized sets of weights are kept for these purposes. In Java the Dutch merchants resorted to the same expedient to cover both wastage and loss. The King of Siam had 'royal weights' when accepting customary tribute in kind from his subordinate kings and subjects; i. e. he received at a different weight to that which he parted with the goods so received. All this is merely a way of maintaining buying and selling prices, and raising revenue and taxation adapted to surrounding circumstances.

Lastly in this connexion I will adduce some evidence from Turkestan in Central Asia in the present day, where mulberries are the currency, just as till quite lately bitter almonds were the currency for small values in many parts of civilized India. I have kept it to the last, because the story thereof carries me to my next point and affords a parallel to what I have said of the Nicobars. Quoting from a recent Russian Report we are told of Darwaz, which is in Bokhara, that 'the inhabitants of Darwaz plant mulberry trees and the mulberry is their sole means of subsistence. In summer they eat it raw, and in the winter in a dried state in the form of flour, out of which they make a kind of flat cake. Their dress they obtain by bartering the mulberry for 
rough matting and sheep-skins, and even their taxes are paid with the mulberry. In fact the mulberry is the measure tubeteika, the currency of Darwaz, and many Darwazes never know the taste of bread all their lives long. ... The grain measure is the batm $\bar{a} n=45$ tubeteikas.'

The value of this bit of evidence consists in the fact that when the dried mulberries were made up into tubeteikas or measures, the tubeteikas became conventional articles or tokens. This indicates the next crucial advance in reasoning. When we make a purely conventional article, i.e. a token, usable only as a medium of exchange and for no other purpose, we have set up a system of money, for money consists of tokens convertible into property. So many imitation, not real, spearheads can buy an adze: so many an axe. Money is in fact currency of a particular sort, and the progressive reasoning that has brought it into existence is this. Barter is the exchange of one article for another, currency implies exchange through a medium, money that the medium is a token, i.e. something of no domestic use, conventionally made and not at all necessarily of metal.

The state of transition from currency in kind to currency in money is so remote from the conditions in which modern English people have been brought up, that its existence is not readily to be grasped. It constitutes, however, an advance in reasoning so decided that the tracing of the conditions under which it arose is beset with difficulties. Here the fundamental law to be observed in all anthropological research, which I have so far pointed to as merely a matter of observation, becomes a law on which our deductions have to be based, i.e. of the first importance. It is the Law of Contact, which 
I took the first opportunity that offered to-day of insisting on.

The cause of all the difficulties that beset our investigation now is the fact that every section of mankind in every place and at every period, being a product of Nature, has never developed along a single line: Man has always been subjected to and affected by outside influences. He has picked up a little here, snatched a little there, and engrafted what he has caught up on to the tree of his own ideas, with the result that its subsequent growth has become complicated or even diverted from its original tendency. No semi-civilized group of men has been at any time entirely isolated, and in tracing the development of currency anywhere, the influences of contact with the outside world are everywhere and always more or less plainly apparent. Barter is the natural basis of all dealings between man and man, and the setting up of a common useful article as a medium of exchange is a natural development. But somehow a community under our observation has learnt to count after a fashion. Somebody has taught it how to measure, or in some forgotten way it has been led on to a distinct point further in upward development and has acquired the art of measuring by weighment. Whenever this has happened, and one or more or all of these things have happened nearly always to any community we can study nowadays, complications have ensued. 'The result is, of course, that in any given concrete instance of barter, it is not by any means to be clearly separated from currency, and vice versa. You will have already perceived this in the course of my present remarks. It must have occurred to you that some of my illustrations of barter are perilously near currency, and that the aptness of some of my cases of currency is 
jeopardized by their close approach to barter. Just so. In my view, in illustrating by examples a natural development, this is inevitable. It is a phenomenon of Nature, of which the explanation I offer is that just given.

Bearing this law always in mind, let us commence our investigation of the borderland between Currency and Money, between the employment of a domestically usable article and the employment of a domestically nonusable article as the medium of exchange, by a consideration of roughly measured articles as the medium. In the fourteenth century Friar Odoric tells us of a rich man's revenue in China being stated in sacks, i.e. 'heavy ass-loads' of rice, revenues there being, until quite lately and perhaps still, estimated in sacks of rice. In Burma under the native Government they were always reckoned in baskets of rice, just as they were in Kashmir in the sixteenth century in the days of Akbar the Great. All this is on the same principle as the use of the rolls of tobacco with which the young Virginian paid for his bride's passage out from England, though the measuring is not, owing to the comparative civilization of the parties concerned, so accurate or regular. In the same category should be placed the well-known currency in skins in Ancient Russia, North America, and China.

From a roughly measured currency to a carefully measured and, so far as regards measurement, regulated currency is an almost imperceptible step. Of this the tobacco rolls of Old Virginia are equally as much an example as they are of roughly measured currency, giving an idea of the difficulty in some cases of arriving at a distinct attribution to class in the case of natural phenomena. The tubeteika is, however, a clear instance: Forty-five tubeteikas, or mulberry cakes, make by local law or custom a batmān or standard measure. And 
when we come to study our old friend Marco Polo's sayings about ' Tebet' in the thirteenth century we find the same thing: "The small change is made in this way. They have salt which they boil and set in a mould, flat below and round above, and every piece from the mould weighs about half a pound. Now eighty moulds of this salt are worth one saggio (say a sixth of an ounce) of fine gold, which is a weight so called.' In other words, eighty moulds of salt of a definite size made a liang or Chinese tael of the period. The experience of the Dutch in the Malay Archipelago in 1596 was much the same in the matter of cakes of sago. In I 7 Io Alexander Hamilton, the traveller, found bees-wax in oblong moulds used as currency in Borneo. These had a fixed weight of a quarter pecul of $34 \mathrm{lb}$. and a fixed value of $2 \frac{1}{2}$ mace, or ten shillings in gold. Tea in bricks and cakes is another similar form of currency in natural produce, which has been widely noticed by travellers and others, and has what naturalists call a wide distribution, for it is found from Shanland and China to Russia. The use of tea-bricks and their close approach to money is well put by the traveller Baber, who wrote in $188_{2}$ that 'a brick of tea is not only worth a rupee, but in a certain sense is a rupee.' Some twenty years earlier Clement Williams, a name once well known in Burma, wrote: 'The only kinds apparently known in the market at Bamo are the flat discs of China tea and the balls of Shan tea. The discs weigh 20 tickals each: seven piled together make a packet, which used to sell at $1 \frac{1}{2}$ to 2 tickals (of silver, say 5 shillings).' These parallel instances from widely differing regions are excellent evidence for our purpose.

The next progressive step is an easy pass from the use of natural produce in conventional cakes, bricks, balls, disks, and what not, to articles that are entirely

s. I. 
manufactured. There is for the present discussion a valuable reference to a currency in cloth in a letter from John Jesse, dated July 20, I775, to the East India Directors. This old Oriental worthy writes: 'I was informed the quantity (of pepper) that year ( 1774 in Borneo proper) was 4,000 peculs, cultivated solely by a colony of Chinese settled here, and sold to the junks, at the rate of 17.2 Spanish dollars per pecul, in China cloth called congongs, which for want of any other specie are become the standard for regulating the prices of all commercial commodities at this port.' This congong then must have been a piece of cloth of an average length and size, and therefore it belonged to the category of carefully measured articles, domestically usable and employed as a medium of exchange. In just the same way a piece of cloth, highly ornamented with shells, passed as currency in Formosa.

A similar currency, both old and widely distributed, in the Far East, in Burma, Yunnan, Shanland, Siam, Malay Archipelago, and Borneo, among other places, consists of glass jars and bottles. The Chinese noticed this of the Burmans a thousand years ago, and in 1870 and 1874, during expeditions in Upper Burma, one writer notes that 'what money could not secure empty pint hock-bottles could. For four of these I got eleven eggs and a brood of jungle-fowl chickens.' Another noticed that the Shans placed 'an inordinate value upon empty bottles.' Any kind of liquor bottle was good, soda-water bottles were better, red hock-bottles best of all. In the Consular Report from Yunnan for 1898 we are told that in the hills these 'bottles are accepted in preference to silver,' the bottles being 'good quart bottles of clear glass.' Here we have a ready-made careful measurement, which the users of the currency 
are unable to effect for themselves, though they are thoroughly alive to the value of the constancy in the size respectively of the hock, soda-water, and other bottles. Of the same nature were the beautifully made arrows which were the currency of the Torres Islands in the South Pacific.

De Morga, the famous and exceedingly intelligent Governor of the Philippines, early in the sixteenth century, gives us yet another valuable instance, for after explaining that the usual way of trade was in general barter, he says: 'Sometimes a price intervened, which was paid in gold according to the agreement made; also in metal bells brought from China, which they value as precious ornaments. They are like large pans and are very sonorous, and they strike upon them at their feasts, and carry them in the vessels to the wars instead of drums and other instruments.' We are here still in the region of a currency of the same sort precisely as the glass bottles of the Shans, but when we come to look into the story of the big drums of the Karens of Burma, of which two fine specimens are in the British Museum, the conditions are much less clear. Of these we are told by an experienced writer that 'among the most valued possessions of the Hill Karens is the kyee-zee, consisting of a copper or spelter cylinder of about a quarter of an inch in thickness, averaging about two feet in length, and of somewhat greater diameter at one end, which is closed with the same kind of metal, the smaller end being left open. They are ornamented in a rude style with figures of animals, birds, and fish, and, according to size and volume of sound, are valued at from $£_{5}$ to $£ 50$. On the outer circle are four frogs. They have distinctive names for ten different kinds, which they pretend to distinguish by sound. In the settlement of 
their quarrels and in the redemption of captives, the indemnification always takes the shape of a kyee-zee or more, with a few buffaloes or pigs as make-weights.' To such an extent does the passion for the possession of these instruments predominate among the more secluded tribes, that it is said that instances are by no means rare of their having bartered their children and relations for them. The possession of kyee-zees is what constitutes a rich Karen. No one is considered rich without them, whatever may be his other possessions. Every one who has money turns it into kyee-zees, and a village that has many of them is the envy of other villages, and is often the cause of wars to obtain their possession. The Karen drums then are of varying size, are used in making large payments, and represent wealth. If they are put to domestic use, as for feasts and what not, they must be classed as currency: if they are to be looked upon merely as tokens of a certain value, and kept only for making large payments when due or only as representative of wealth, then they are money. They are, in fact, just on the border-line between currency and money. They do not stand on the borders alone, for the use and value of the great war-shields of Guadalcanar in the South Pacific do precisely the same.

Also, Pyrard de Laval in I60I tells us of Angola: 'As for the small money of Angola, it consists only of little shells, somewhat like those of the Maldives (i. e. cowries), and little pieces of cloth made of a certain herb. These pieces are an ell in length, more or less, according to the price. And when they go to market to buy their goods they carry no other money.' Now, if these ells of cloth were for personal wear, they were thus used for currency: if they were, as I understand, never worn, they were made for money. Even to these there is an 
exact parallel in the strings and beckets of flying-fox hair and in the tridacna shell-ornaments of the South Pacific.

The progressive reasoning that has converted currency into money has thus gone very far, but it is necessary to go further still before the momentous transition can actually take place. From Yule's edition of Marco Polo we learn that on the salt-moulds already alluded to 'the Prince's mark is printed, and no one is allowed to make it except the royal officers.' In Russia, tea-bricks have been stamped with something like a Government or official mark. In these instances we have reached something not only very like money, but even corresponding to stamped money, which two terms, as will be presently explained, are not at all synonymous. But as both the salt-mould and the tea-brick can be put to an ordinary domestic use they are still currency and not money. Here, again, the law of the constancy of human reasoning comes prominently into view, for the reasons that led to the stamping and officially marking the tea-bricks and salt-moulds are precisely those, as we shall see presently, that have led to the stamping and marking of coined money.

A clear instance of the exact point where currency ends and money begins is the use of rice as a medium of exchange in Burma, existing in my time there about twenty years ago, and noted also by the Resident at the Court of Ava in 1797. It was used in village transactions in Upper Burma, but such rice was neither food-rice nor seed-rice, but useless broken rice. In other words it was a non-bullion token and so money, just as the imitation hoes, hatchets, knives, and so on of the Chinese and other peoples in various parts of the world are tokens of currency, and so money. Another almost 
universal instance of a non-metallic money proper is the cowry; for these sea-shells, where chiefly used in the East, are not of any domestic use whatever to the people who pass them from hand to hand, and are expressly imported in very large quantities, often from great distances, only for the purpose of a medium of exchange. They afford a clear example of an untouched natural product being converted into money as distinguished from currency.

We have now effected the great transition, and having passed into the region of money are face to face with a fresh natural law-the Law of Money-which will follow us through all developments from the earliest to the latest. For all these things-broken rice, imitation iron instruments, and cowries-properly fulfil the conditions for money. They have to be produced, and though fairly common, the production is, in the conditions in which the producers live, nevertheless limited, and therefore they can have a token value. To take the least likely instance. Rice has to be cultivated; the amount of cultivation depends on the capacities and numbers of the cultivators; of what is thus produced a fixed quantity must go for food and another fixed quantity for seed; only what is spoiled in handling and what is over can be used for money. The production of broken rice is therefore distinctly limited and at the same time sufficiently abundant. This is why to isolated half-civilized villagers, living in certain places, broken rice is money. The reasoning that makes it money for them is precisely that which makes gold, silver, and copper tokens money for us.

Another example of all this is a feather ornament in the New Hebrides, which is worn by men after celebrating the prescribed number of feasts and then used 
as money, thus showing how an article which has been domestically usable passes into the class of articles domestically non-usable on becoming money. There is also the tusk-shell money of British Columbia, consisting of clipped dentalium shells in strings of eight sections, divided by bars of goat leather, with a pendant of the haliotis or Venus's ear. The shells are procurable only under certain conditions, which fact limits their production, and strings of them become money for precisely the same reason as the bags of broken rice of Burma. The whole of this important point is further illustrated admirably by the honey-sucker feathers of Hawaii, which are stuck in bundles on strips of coco-nut just as they are collected, and are currency, because in this state the feathers are used as a medium of exchange, but are also used for ornaments, clothing, and other domestic purposes. They well indicate, nevertheless, the origin of the use of natural products as money, being plentiful and yet limited in production. The limitation was due to the fact that feather-hunting was a vocation. The feathers, too, had a relative value according to rarity or difficulty in production. Thus five yellow feathers of the 'royal bird,' which were all that the bird could produce, were accounted equal in value to a piece of nankeen, which was sold for one and a half dollars. This would probably represent to the natives at least a pound of our money.

The Law of Money then is this. An article to be usable as money must be fairly common; it must be produced, and its production must be limited. And in order to trace the development of money further, we cannot too clearly perceive the difference between currency in kind and currency in money. The former implies the use as a medium of exchange of a domes- 
tically usable article, and the latter of a token not domestically usable. It is this last consideration that is the test by which we can separate metal articles, when used as a medium of exchange, into those that are currency and those that are money. When the ironsmelting Shans of Zimme pay their revenue in elephantchains, spear-heads, cooking-pots, and other iron-work which they make, they are using currency: but when similar Shans along the Mekhong use lozenges of ingot iron for making payments, they are using a real money. So also the usable iron hatchets or handbills of the Nassau Islanders, found in use in 1792 , were currency, while the thin, i.e. imitation and useless, iron knives of the Kachins and Shans of the Assam-Burma border of about the same period were money. Thus, too, the gold and silver boxes, bowls, and necklaces, and the quainter and prettier gold and silver flowers, leaves, and even trees of the former Shan, Malay, and Burmese tribute formed a sort of currency and not of money. The evidence is universal. The imitation knives, hoes, and razors of China made of spelter, iron, and copper were money, as were and are many metal articles among the African peoples. Such were the Lomami and other spear-heads, the plaques of iron, the copper ingots in accidental crosses in Central Africa; the imitation iron adzes, axes, axe-blades and heads, spears and hoes, and the copper, brass, and iron bracelets, known as manillas, with a long descent historically, in the West; the magnetic iron hoes in the East.

On the other hand, true currency is shown in such articles as the following, which were used domestically as well as pecuniarily: the hammered brass frying-pans of the Nagas of Assam; the salt cylinders of East Africa; the salt bars, quills and bags of gold dust, and rings of 
virgin gold of Abyssinia; the iron hoes, spades, and barbed spear-heads of Central Africa; and the brass and copper plates and bar-iron of North America. Parallels to these are found in the necklaces of red feathers and of shell disks, and in the jadeite adze-blades of various Pacific Islands.

From all parts of the world, too, comes evidence of the use of many kinds of local productions by savages and rudely civilized peoples for the same purposes as we use coined money, and of these productions having a relative value for the same reasons as the metals have amongst us: i. e. relative difficulty as to production or relative rarity, showing once more the law of the constancy of human reasoning, and in the case of the modern savages the Law of Contact. As money there have been used in the Southern Pacific Islands strings made of the fur and even of the lower jaws of the flying-fox, and of disks of shell carried in a purse, and the well-known Navalae rings, which are made of white quartz. The Californian Indians use the hawock money, consisting of clam shell disks in strings measured by the foot and yard. Relative values are clearly shown in the toothmoney of some of the Pacific Islands, where certain teeth of the dog, i. e. the rarer and more difficult to procure, are worth from two to five porpoise teeth. Very high value, owing to slowness and trouble in production, is shown in the large bands of parrot feathers of Santa Cruz.

The well-known existence of the use of beads as money covers the entire ground of monetary values. The point to get into our heads here is that beads as beads do not appeal to the savage and semi-civilized man for any purpose other than ornament. As money they appear to him as a mere question of kind and value, 
i. e. of rarity or difficulty of production. For instance, take the brack money of the Pellew Islands in the South Pacific. Here we have highly finished terra-cotta beads, very valuable; jasper, agate, and enamel beads, valuable ; green and white glass beads in four grades of relative, but low, values. Then we have strings of small, highly finished shell disks, fifty-four to the inch, in the Banks Islands, of very high value; while beads of sorts made of mother of pearl, red and other stones, nutshells, tortoise-shell, and coco-nuts bear low values. In exactly the same way the Venetian and Aggry beads, with their long and ancient history, and other beads bear in Africa ever varying values proportionate to kind and temporary rarity, as do also the blue glass beads of the Kaffres of South Africa. The wampum beads of North America, in what may be called their natural state, bore a relative value of one purple to two white, owing to relative rarity, and in their manufactured state imported from Europe they bore a value in strings by the inch and fathom dependent on cost of production and importation, or, as we should say, on their intrinsic value. On the other hand, the 'dashes' or beads for ornament of West Africa have no value as money.

Mankind has thus arrived at the notion of money by a very slow process step by step, and by the use locally of any articles, natural or manufactured, that happened, for the place and time to satisfy the conditions of a medium of exchange-abundance, production, limitation. These articles have been of any nature, and among them the metals and articles made of metal have had to take their chance of general acceptance with the rest, and have come to the top and outdistanced all the rest among civilized peoples merely in obedience to the great general natural law of the survival of the 
fittest. And among the metals, what we call the bullion metals, i. e. gold and silver, have come to be generally used for money, and currency has come to be expressed in terms of gold and silver money, only for the reason that civilized man has long found out by experience that these two metals are the most convenient materials to be found on the earth as media of exchange. The instability of a metal as a medium of exchange and money is shown by the general supersession of copper by nickel for low values in our own time, and firmly established as gold and silver may seem to us nowadays to be, they will be most certainly discarded the moment anything more convenient turns up for general use as money. In fact gold is showing a tendency to supersede silver and to become the only currency metal, just as silver superseded gold in many places for many centuries and still supersedes it, and is and was the only currency metal for those places.

Modern commercial and financial men among the highly civilized recognize this fact by another name and talk about the 'gold or silver basis': but the meaning is the same, and as surely as gold or silver, or both together, in a constant or a not too varying ratio to each other, form the most convenient medium of exchange, so surely will one or the other or both be money. In modern parlance, so surely will monometallism, in gold or silver or bimetallism in both obtain. It all happens in unconscious obedience to the natural law, already insisted on so often, of the constancy of human reasoning and the action based thereon.

Having arrived at the crucial point of measuring the relative value of possessions by means of the media of exchange, the next natural mental process is to express that measurement in terms of the media. That is, when 
we describe wealth in pounds, francs, marks, dollars, rupees, and so on, we are expressing it in the only common denominator known to us-the name of the measure which is the medium of exchange we use. That is why to say that a man is worth, i.e. his possessions could be exchanged for, 10,000 francs or rupees or marks, conveys no meaning to an untravelled or not specially educated Englishman until the terms are translated into pounds, the name of his standard of currency. The same argument applies to the 'worth' of anything stated in terms of any currency, metallic or other.

The first step towards metal money is, of course, the use of a lump of metal as currency, i. e. of a manufactured article produced from the ore by smelting, but of uncertain quantity or weight and of most uncertain quality, or fineness resulting from the mode of smelting. In this condition of affairs, which it is not easy for the modern Englishman to realize, every piece of metal tendered in payment or exchange requires weighment and examination for fineness or assay. This was, however, a common condition all the world over for a very long time, is so still in many places, and was so in Burma until quite recently. It was what our forefathers meant by the old expression of 'showing your money.' It obtained in Upper Burma in my own time. In fact it was the interest aroused by observing how people managed to flourish financially and trade in such circumstances that led 'me on to take up the subject of currency as a study. I found that for their daily wants people living under these conditions acquire dexterity in weighing by the hand and in recognizing the quality of metal by appearance and feel. I did so to a certain extent myself in the case of silver, the metal I studied. Here again mankind is seen acting in obedience to a natural law-that of 
adaptability to environment. Nothing can continue to exist or progress except in obedience to this law, which is the root of all evolution.

The practical usefulness of weighing by the hand is limited to small pieces of metal, as payment or part payment for continuous and customary purchases and sales between the same parties. But it is obvious that lumps of bullion of uncertain weight, i.e. size, cannot exactly pay for small articles occasionally or sporadically bought. This difficulty has been naturally settled by chipping pieces off a larger lump to satisfaction. In China, Burma, Tibet, and many other places, chipped bullion currency largely obtained, and in places still obtains, a hammer and chisel being kept by the more skilful and resort to the nearest jeweller on payment being had by the unskilled for the purpose. I have myself possessed such a hammer and chisel. Sir Frederick Younghusband resorted to jewellers for chipping during his great journey through China. Again, where actual coined money has been looked upon as mere bullion the larger denominations have been chipped to make the smaller. The best instance comes from the Himalayas, where the octagonal Nepalese rupee, each part of the octagon containing the impression of a flower, is split up by chipping and cutting into 'flowers' or eighth parts, especially in the neighbouring state of Bhutan. A parallel showing the constancy of reasoning occurs in Abyssinia, where the salt-bars used as currency are 'broken' to make small pieces.

Here then is the next step towards metal money, the possession of pieces of metal of accepted weight. When $x$ these pieces have been weighed in scales, a process which has naturally followed, as is proved by the universal existence of jeweller's scales, the chipped pieces 
of bullion have become tested for weight. So greatly has the necessity for the weighment of bullion impressed itself on mankind that the highly civilized Eastern mind is still largely incapable of separating the ideas involved in the terms used for money and for its weight, and in many parts of Europe, though we count our money, we still speak of it in terms of weight when naming our money of account: e.g. the English pound. Like everything connected with money, this very counting of pieces of it has a development of its own, which can only be alluded to in passing, though it is well worth exhaustive study. The whole history of tallies and tallying is of interest, especially where, as in the case of the Nicobarese, it is possible to trace in one people the entire evolution from the rude tallying of their currency in pairs of coco-nuts to the use of practical, but entirely unconscious, commercial scales based on enumeration by the score, which by the way is itself the decimal scale applied to pairs. Then again there is the method of counting cowries by separating them out of a heap in fours and fives, leading to the well-known and important Eastern scales of quintets and quartets and of four fours or sixteens. The adaptation of quartets to an alien currency, superseding their own, has led to perhaps the most complicated monetary nomenclature in existence in the State of Manipur on the Assam border, just as the clash and semi-amalgamation of the great Indian scale of sixteen and of the Chinese decimal scale (which in passing it may be noted is not indigenous) has led in Burma to that apparently but not really hopeless division of the unit of money that has induced the people to speak of six where they should say five and of five as the half of eight. We have also had in Europe our cambists, which deal with almost every conceivable scale 
for reckoning money. We have further the old dozen or duodecimal scale with a most ancient descent, so convenient in dealing with small quantities and, unlike the decimal scale, so inconvenient in the case of large ones, whence the prolonged current controversy on their respective merits. And lastly, we have a whole gamut of scales in the Far East developed out of those prevalent in the Middle Ages among the Malays on the commercial high water-way from East to West, which in themselves are probably all of Indian origin: and the modern Indian scales representing developments side by side of indigenous scales and those imported originally from Europe.

The idea of counting currency has also, wherever it has existed, led at once to those of interest, or return of more than one lends, i.e. gives temporarily, and of capital which is the accumulation of separate pieces. It is not at all necessary that metal money, or even easily divisible or separable money, should exist to turn it to personal pecuniary advantage by way of interest or capital. In the South Pacific islands there is the matmoney, consisting of mats made in great lengths in folds, whose relative value is determined by the number of folds counted in tens and their blackness or age. Mats with fewer or newer folds are lent in return for mats with more or older folds. Here is the whole theory of interest, and when these mats are accumulated we arrive at capital. In the same way masses of tapa or black cloth money in Fiji when accumulated represented capital. In these cases we have strong instances of the law of the constancy of human reasoning.

To go back to the lumps of bullion. A piece of bullion metal whose weight only has been tested is still, though capable of being counted, an uncertain medium 
of exchange until its quality or fineness has also been tested. An instance of accurate testing by natural form and appearance for daily use occurs in the silver shellmoney of the Shan Native States of Burma. The form of the snail-shell taken on by this money is held to be partly artificial thus: Silver refined by the ordinary process in a rough crucible will, when very nearly pure, or what the natives call quite pure, effloresce, and if the efflorescence is checked by cold water at the right moment it will assume the shell form: So silver in that form is looked upon as pure and the silver shells pass as currency by weight. A parallel in a non-bullion currency is found in Abyssinia, where the people test the bar-salt currency for quality, i.e. assay it, by placing the bar close to the ear and scraping the finger-nails along it. The quality, i.e. the damage that may have been done to it, is ascertained thus by its ring, just as our people will Xring coins for rough assay. Here the constancy of human reasoning is very strongly perceptible. Assay by appearance applies, too, in the case of the very highly civilized invention of paper money or bank notes where it is commonly used. In such countries an old note, like the savage mat-money, passes better than a new one, as being the more likely to be genuine, because many people must have used it as such.

Instructive as these instances are of the development of thought and manufactured form, personally testing the metal usually offered as money in the course of dealings by the eye or ear or finger was too vague an operation in the case of important payments. Resort by ordinary people in such cases would naturally be had to an expert examiner or assayer, and this necessity has led to an extensive profession in the East, and indeed it may be said everywhere, of assaying bullion for the public, 
that has played a most important part in the economy of life. In this matter the money-changers at the Temple of Jerusalem were but the counterparts of the still living money-changers on the steps of the great Shwe Dagon Pagoda at Rangoon.

The processes of weighing and assaying lead necessarily to the creation of standards, as the results both of weighment and assay can only be stated in terms involving a comparison with something. One of such standards of weight has been the seeds of some plant, which have been found by observation and experiment to possess a fairly constant weight. The widely distributed bright and eyed seed of the pretty creeper Abrus precatorius is an instance. For the commonest standard or fundamental starting-point of bullion weight, or as we call it in England troy weight, is in the East that very variously known as the rati and gunja in India and the yze in Burma, and as the mangelin and candareen in Malayland and thence in China by European corruptions of nomenclature. Every one of these is a name for the same things, the abrus seeds, which are further known as starlings' eyes among the Malays, cocks' eyes in Persia, and King Charles's tears among English children in the East, attesting their universal use there. In expressing the weight of any given piece of metal the habit arose in the East of stating it in terms of these seeds, and a standard of weight was created.

The usual standard of assay has been the purest gold or silver that could be procured by the processes of refinement in vogue, and fineness has thus been expressed in degrees purporting to exhibit the amount of alloy or foreign substance in the piece of gold or silver spoken of. A knowledge of these degrees has been arrived at primarily by direct assay through the melting- 
pot. By this process the colour, i.e. the outward appearance of a piece of gold especially, but also of silver, is ascertained by actual experiment, coupled with a knowledge of the comparative amount of 'pure' metal and of the usual alloys put into the pot. By rubbing the specimens thus procured on black 'touchstone ' in streaks side by side with specimens of pure gold or silver we obtain the 'touch,' i. e. the difference in colour between specimens of the pure metal and the alloyed. The touchneedles used by the Oriental jewellers in ascertaining 'touch' are but a set of graduated specimens of metal tested for the alloy in each.

When we have progressed as far as this, we have secured possession of lumps of metal of a known weight and a known fineness, and have made a distinct step forwards, for we have arrived at a coin in its simplest form. In many parts of the world, and in the East, especially among the poorer classes, any piece of bullion metal - a term there extended to gold, silver, copper, tin, brass, spelter, and even to lead and iron-is still used and passed from hand to hand as money without reference to any marks thereon, provided its weight and fineness are known, or, what comes to the same thing, are accepted. It is, in fact, for these people a coin. In Burma, disks of silver, copper, and brass which have come from the old native mints accidentally unstamped, and in India brass English jettons or card counters with legends thereon showing that they were not intended for what we call money, have passed, and no doubt still pass, with equal freedom as money, side by side with coin of the realm for the value of the metal in them. So have metal buttons and filed coins, and of course obsolete coins of any kind and period. The magyizi or silver imitations in Upper Burma of the tamarind seeds 
used as royal playthings in a popular pitch and toss game, passed lately as money, because of their constant weight and fineness.

The next step in evolution is taken quite naturally. Weighing and assaying lead directly to marking for identification. When all money is vague and requires testing by both weight and assay before use it is natural to put a recognizable mark on any particular piece one knows. It saves so much trouble. The first move in this direction was to mark lumps of silver for fineness. It saved working out that troublesome point every time they came into use. The best known instance of this is the famous sycee silver of China, all of which is stamped for fineness, but not for weight, by private Chinese firms, $x$ the Easterlings of the East, with characters very widely recognized. Others are the larins of Persia and west coast of India and the 'fish-hooks' of Ceylon, which both consisted of silver wire bent and stamped for fineness, and the ticals of Siam, which were bars of silver hammered double and similarly stamped.

By this process, however, we have only arrived at a mark for fineness, but it is necessary to know both fineness and weight. The 'chopped' dollar of China is a well-known instance of the first in this direction. The dollar, being an actual European coin found by the Chinese merchants to be of constant weight, they have acquired a habit of accepting its weight, but their old instincts, or perhaps unfortunate experience, have induced them to 'chop,' i.e. stamp not cut, for fineness, every dollar that passes through their hands and with which they are satisfied, so as to recognize it. In this way dollars have become stamped all over till it requires some skill in the users to pick out their own mark, for they will accept no others, including the European marks. 
In the 'chopped' dollar then we have an instance of the full-blown natural coin, or a piece of metal marked or known both for weight and fineness, i.e. exchange value, by its shape, size, and the marks thereon.

But we have not yet arrived at what we all know in Europe as money, for that term is not applied to any coin as just defined, but only to the coin of the realm. Every country has its own money and recognizes all other coin as foreign money, putting an altogether different value on each. The value of the coin of the realm is its assumed conventional value by law, and that of all other coin is its value as bullion in comparison with the value of the coin of the realm as fixed by law, or as it is ordinarily put, all foreign coin is liable to exchange, a term which will by now be quite understood. This has been brought about by natural processes.

Weighing, assaying, marking, and stamping by private persons specially employed by the public lead directly to what is called roguery, i. e. to the unprincipled use of that instinct of trade, so strong in man, of profiting by the necessities of his neighbours and taking advantage of any ignorance they might possess. In all ages, therefore, and in all places, the constituted authority has been obliged in the interests of internal peace to regulate by law both weights and assay by fixing standards of each. But even when the weights and assay of coins have been fixed by law, the final point has not been reached, until the legally tested coins have been stamped or marked to show that they are legal currency.

Very long ago it was recognized as one of the highest and proudest duties of the strong ruler to preserve the purity of the coinage of his country as a medium of exchange, and hence the pride taken by kings in affixing their own effigies and inscriptions thereon as a guarantee 
of its exchange value: a habit that has so grown with time as to become an intuitive custom applied to adhesive stamps or any other article which has a legal value. So widely have the marks of certain rulers become known and so greatly have they been appreciated that other peoples and kings have copied them after a fashion, and in some instances they have had to be continued after death.

The spread and imitations of the coinage of Philip of Macedon are instances in Europe. The issue of the rupees and gold mohurs of the Mogul Emperor Shah Alam by the East India Company and others long after his death, and the use of the Hindu Devanagari legends on the coins of the early Muhammadan invaders, only gradually giving way to Arabic and Persian, are instances in India. The wide use of the Spanish and Mexican dollars in the Far East and of the Maria Theresa dollars in Abyssinia are yet others. Where for local reasons it has not been practicable to establish a stamped or engraved or otherwise certified coinage, or where perhaps its introduction has not come to a civilized people as a development, its value has even in ancient times been at once recognized when pointed out. When some Greek travellers in the centuries B.c. visited Taprobane, which we may assume to be Ceylon or the west coast of Sumatra, the king of the country was so greatly struck with the practical use of the gold legally stamped coins exhibited to him that he lamented his inability to introduce them into his own country.

Of course deceit and fraud, i.e. the improper use of knowledge or power for personal advantage, are not confined to traders, and long ago and everywhere every kind of device has been adopted for what is called debasing or forcing the currency by both traders and 
rulers. But so vital is the constant value of the currency or coinage to the daily welfare of all communities that no amount of trickery and no amount of force can permanently affect the value put on the quality of the medium of exchange. There is one thing that a smart mercantile body or an autocrat or tyrant cannot do. They cannot permanently alter the quality or weight, i.e. the exchange value of the medium of exchange. As soon as they are found out their money 'falls,' as we say, to its intrinsic value. Close and clever imitations of the very valuable blue Popo beads of West Africa, which are probably made of beryl or aquamarine, have never yet been passed for true ones on the savages there. In the seventeenth century smart Chinese merchants imposed very debased, but bright silvery, spelter coins of exceedingly low value, which we call 'cash,' on the unsuspecting Malays, but when the trick was discovered their value went down to a thirty-thousandth part, i.e. to practically nothing, showing the result of playing with currency when dealing with the semi-civilized. It has never recovered since beyond the hundredth part, as its name sapeque in French still implies. In the East in the same way constant and continuous royal debasement has caused denominations to decrease steadily even to a thousandth part of their original value. The mahmudi is an instance, receding from $£ 4$ to a penny in say six hundred years. The East India Company in the eighteenth century tried to introduce a gold mohur containing fifteen annas in gold for the value of sixteen annas with a complete want of success. Oriental and other autocratic kings have repeatedly tried at the point of the sword to force a debased coinage on their subjects, but have never succeeded. In Burma the apparently irresistible old autocrat, Bodawgyi, a hundred years ago, 
tried to impose a brass coinage at the value of copper on his unfortunate people by an enforced death penalty, but to no purpose. He found that killing individuals as examples could not bring the community to face general commercial ruin to his advantage.

We have now reached our goal, the coin of the realm proper-the piece of metal with a fixed legal exchange value, shown by its shape and the marks thereon attesting its weight and fineness, i.e. its exact adaptation to its purpose. By its very shape an English sixpence shows its evolution, for the roundness has been arrived at after painful efforts to try the effect of every formoblong, oblate-spheroid, square, octagonal, hexagonal. For every other form will cut the bag or receptacle that holds quantities, and square corners will get damaged by use, causing reduction in weight. The milled edge prevents fraudulent shrinkage by stripping off and reducing the rim. By sinking the whole die-stamp lower than the rim specimens can be piled indefinitely on each other, or put away in rolls occupying the smallest practicable $X$ space. The holes in 'cash,' and through the cash in such very modern coins as the nickels of Belgium where the hole is used merely for differentiation, have an old descent from the hole in the handle of the conventional 'knife-money' of China, which permitted articles for currency to be strung together. All this represents a very slow evolution. In very fact English sixpences, French francs, and pieces of any other similar money one can mention, the uses to which they are put and the manner in which they are used, are all, like their owners themselves, in obedience to the natural law of heredity, heirs of all the ages.

Before closing these remarks I will briefly note one or two points of some importance, as to which confusion 
and mistakes may easily arise. In I 24I the Emperor Frederick II, son of Barbarossa, issued a temporary and honest leather currency. In the last century, among other places for local reasons, parchment and paper currencies were temporarily established respectively in the Cocos-Keeling Islands and in the Andamans. From the ninth to the fifteenth centuries a most remarkable paper currency was very widely established in China. For a long time past there has been, and probably still is, a noticeable currency in porcelain gambling tokens in Siam, analogous to which were the tradesmen's tokens of England and Europe, and the taungbanni, or private mint money, of Burma. Now, not one of these has any connexion with the state of things we have been considering, for their existence is dependent on conditions which are different altogether, as they are each and all based on commercial credit. So also the modern English silver money, the nickels of the countries using them, the Indian rupee, the Japanese yen, the United States dollar, are really tokens and money $X$ of account only, being taken, as coins, to represent more than their intrinsic value in terms of the gold standard on which their value is based, but this fact arises out of very different considerations than those we have been discussing.

Then again there is any amount of evidence to show that oppression and cheating are just twice as easy with a non-bullion currency as with a legal standard of gold and silver coinage, which is in reality, so far from being a curse as is so often preached to us, one of the greatest blessings that man's ingenuity has ever brought about for the benefit of his kind. In this connexion I may once more draw attention to the anthropological law of trade adapting itself to any circumstances. Cowries, 
where used, are bought by local dealers wholesale by weight in sacks and retailed by tale, so that the smaller the cowries which the retail dealer can manage to pass the greater his profit. In India the cowries of currency are large, on the west coast of Africa they are of medium size, in the South Pacific they are very small. A clear instance of taking advantage of local conditions and the state of public knowledge for personal profit.

Lastly, in such discussions as this the question naturally arises: why have some nations advanced further than others in this matter, the capacity for reasoning being the same? The answer is that advance in any given matter and in any given direction is due to a general advance in other directions, itself due to a thousand causes and very largely to environment. The ability of individuals to advance is strictly limited to environment. The capacity is always there, but it must remain dormant until circumstances permit it to be aroused. It all goes back to the action of the natural law of contact, a condition which mankind has all along unconsciously recognized in the universal proverb that necessity is the mother of invention, and in the term 'opportunity.' In the words of the Preacher in the Bible in remarking on the achievements of men: 'Time and chance happeneth to them all.' Whole groups of communities have observed this, and because of their environment have evolved the unscientific doctrine of Fate and its little suspected counterparts which exist even amongst ourselves. 


\section{ARCHAEQLOGICAL EVIDENCE \\ (Professor W. M. Flinders Petrie)}

THE nature of proof is more complex than it seems to be at first sight. True enough, all proof is merely a matter of common sense; it does not appeal to any different faculty. And though a proof may follow as simply as possible from the facts, yet it cannot be understood by one who is not familiar with the facts to begin with. Trigonometry is the most obvious common sense to any one familiar with the formulae; and the formulae themselves are only common sense to any one who takes the trouble to argue them through. Yet, for all that, trigonometry is not obvious to the ignorant. In the same way the evidences about the past of man are simple and clear when the facts and methods from which they are deduced are already known. Yet it requires a good familiarity with the material before the conclusions can be felt to be self-evident results.

To follow clearly what evidence and proof means, it is best to refer to a class of evidence which is most familiar to the reader. What is commonly called legal evidence is the best-known example, as it is met every day in law cases and police reports. Evidence is based on the same principles, in whatever subject it may be; there is not one logic for the present, and a different logic for the past. But the kind of evidence, the exactitude, the certainty, which is considered enough to determine 
a property or a life, is rightly looked on as conclusive for all reasonable purposes. The laws of such evidence have been threshed over for generations past; and it is well known what kind of proofs may be relied upon, and what are dubious. If we then compare this class of evidence with that which we accept in studying the past history of man, we shall see more clearly what kinds of proof are admissible, and how far it is reasonable to depend upon our results.

In examining legal evidence we see that it all falls under one of four heads-(1) witnesses, (2) material objects, (3) exhaustion, and (4) probabilities. These four kinds of evidence are of very different values; any one of them may be stronger than the others in a given case, and each kind has its own special weakness.

I. Witnesses provide the most clear and connected proof, and the least liable to misunderstanding; but yet a proof which is entirely dependent on veracity, on intelligence, on absence of prejudice, and on clear memory, and is hence the least dependable kind of evidence in some cases.

2. Material facts, which may be very conclusive; such as A's footprint in B's garden, or A's chisel left in B's house, at a burglary. If the fact is certain, the conclusion is proved; but the danger lies in misunderstanding the fact.

3. Exhaustion, which may prove $\mathrm{A}$ guilty because no one else could have done the deed; as when $\mathrm{A}$ and $B$ are seen in a railway carriage at one station, and at the next stoppage $B$ is found murdered and $A$ leaves the carriage. There may be not a trace of other evidence, but this is enough.

4. Probability, as when A is last seen with $B$, and B proceeds to deal with the property of murdered $A$. This 
kind of evidence is enough to hang a man, solely from presumption.

Now let us look at these kinds of evidence about the past of man.

I. Witnesses, the documents, which give a clear and connected statement. They may be either primary, as a stone inscription or an autograph letter; or secondary, as compiled histories or subsequent copies. No other kind of evidence is so easy to follow ; yet this is a proof in which we are entirely at the mercy of the prejudices, the ill-will, the frauds, and the blunders of others, and it is hence the least dependable kind of evidence in some cases. The speeches of Thucydides, the bias of Suetonius, the wonders of Livy, the romances of William of Malmesbury, and the forgery called Richard of Cirencester, each plunge us deeper and deeper into the doubtfulness of written documents; to say nothing of the Casket Letters or Ossian.

2. Material facts, when rightly understood, are the most conclusive evidence. They may be in a single object, as a palaeolithic flint re-chipped over and over in later ages; or a foreign ornament used on an object of dated style, as a Maori tatued head in a daguerreotype would prove the tatuing to be known between 1840 and 1860 ; or a restruck coin with one type over another, as Barchocheb over Hadrian; or an added inscription, so familiar on Egyptian statues. Or the evidence may consist in a collocation of objects, such as a group of things found together in a tomb; or the superposition of strata of ruins in a town. In the case of a single object there are few possibilities of misunderstanding the evidence; but in strata or tomb-groups there is a chance of older things being re-used. Such chances of error are, however, extinguished by the recurrence 
of instances; and the finding of certain things together in several cases under different circumstances is one of the strongest kinds of evidence, such, for instance, as the name of Amenhotep III often found with the Mykenaean pottery, both in Greece and in Egypt.

3. Exhaustion may prove a point; as, for instance, the Iconoclasts in Greece or Reformers and Puritans in England were the only destroyers of images and pictures, or Akhenaten was the only man who erased the name of Amen. Such destructions therefore are evidence of the age and the man.

4. Probabilities, as, for instance, the fact that the Saxons erased the Romano-Britons, makes it probable that Silchester, Uriconium, and other late Roman towns which were burnt, were destroyed by the Saxons.

We see thus that each kind of proof which is accepted legally is also used archaeologically, and is subject to much the same failings. Legal evidence may fail by mistaking the nature of the facts, such as that some rabbit's blood on a knife is human blood; so may archaeology mistake by ignorance, as when the Mykenaean treasure was called Byzantine.

Or legal evidence may fail by wrong inferences from facts, such as that some human blood on a knife is due to a murder, while it has come from the owner's finger. So archaeology erred from a wrong inference in calling the treasure of Troy 'the treasure of Priam'.

Or legal evidence may fail owing to mere prejudice, thus ignoring the truth. So archaeology has suffered from the prejudice that nothing in Greece can be older than the eighth century B.c.

It is supposed sometimes; by those unfamiliar with the subject, that archaeological evidence is so doubtful or so slight that it cannot be relied upon, and is not 
to be compared with the certainties of legal proof. Let us see then what legal proof is in important cases. In one case a will was lost, and the mere memory of its contents, stated by a survivor who had assisted in writing it, was accepted as sufficient proof of what had been in it, and the property was distributed accordingly. In another case property was left by $A$ to $B$, or failing $\mathrm{B}$ to $\mathrm{C} ; \mathrm{B}$ also made a will leaving it to $\mathrm{D}$. $\mathrm{A}$ and $\mathrm{B}$ were killed together in an accident, and the slightest observation of which moved last, determined whether $\mathrm{C}$ or $\mathrm{D}$ had the property. Again, there are innumerable cases of setting a will aside because of the testator not being of a sound mind for disposing of property; and various assertions of irrelevant facts by various interested parties are held to reveal the true mental capacity of a person to a judge and jury. In a murder trial the question of whether one or both of the assailants were guilty was held proved by the deceased having been tied by two different forms of knots. In another trial the mere presumption due to concealing a body and dealing with the property of a murdered person was enough to hang a man. Such are some of the evidences which are held good in law to settle questions of life and property.

Happily archaeology is relieved from the terrible dilemma of being bound to come to a conclusion at once, as the law has to do. Questions can be left pending, and it is not peremptorily needful to act one way or another. An open mind can be kept on difficult and obscure points; and a matter can be discussed in fresh lights, without keeping a prisoner standing in the dock the whole time. Legal conclusions are often wrong; though, as the law can do no wrong, a free pardon is all the sufferer gets when his innocence is proved. But 
if legal proofs, arguments, and conclusions were kept freely open to revision for years; if they were printed in every textbook for beginners; if all students were encouraged to find fresh evidence, and to upset what was laid down, and if the high-road to position lay in reversing the decisions of past authorities, it seems only too likely that there would be a greater wreckage of bad cases and bad law than there now is of bad archaeology.

The earliest stage of history is that of tribal tradition, materialized by localities, relics of past men and events, and mnemonic helps such as knotted cords, group drawings, and the like; as for example among the native peoples of North America. The rhythmical tale or poem, such as the Song of Lamech, Beowulf, or the Sagas, is the highest form of this stage, culminating in such poems as the Iliad.

A register of great events in writing may look back into the earlier stage, as the Lombard history of Paul the Deacon, or the earlier part of the Anglo-Saxon Chronicle. Or it may, like the historical parts of the Anglo-Saxon Chronicle, epitomize what records are possessed. The most remarkable example of this class was the register of about a thousand years of the early history of Egypt, of which some fragments have been preserved to us. This gave the eponym events of each year, and the height of the Nile, the exact length of every king's reign, to a day, and in the later times long entries of the great works of the year. About 4000 B.C. we read in one year of the reign of king Snefru : 'Building a ship of 170 feet long, and 60 ships of 100 feet long. Conquest of the negroes, and capture of 7,000 slaves and 200,000 head of cattle. Building the walls of the southern and northern temples. Bringing in 40 ships of cedar.' 
In the next year: 'Making 35 hunting lodges, 122 cattle tanks. Building a ship of 170 feet of cedar wood, and two such ships of another wood. Seventh cattle census.' Such a system of register lasted on into the monastic chronicles of four or five centuries ago.

The next stage of history regards the connexion of events, and in Herodotus this interest over-shadowed the systematic record altogether. But it is in Polybius that the nature and value of history receives its full place and development. 'Neither the writer nor the reader of history, therefore, should confine his attention to a bare statement of facts; he must take into account all that preceded, accompanied, or followed them. For if you take from history all explanation of cause, principle, and motive, and of the adaptation of the means to the end, what is left is a mere panorama without being instructive; and, though it may please for the moment, has no abiding value.' 'For it is history, and history alone, which, without involving us in actual danger, will mature our judgement and prepare us to take right views, whatever may be the crisis or the posture of affairs.' The original documents were diligently studied, such as the treaty of 509 B.C. between the Romans and Carthaginians. 'Of this treaty I append a translation, as accurate as I could make it,-for the fact is that the ancient language differs so much from that at present in use' (about I4O B.C.), ' that the best scholars among the Romans themselves have great difficulty in interpreting some points in it, even after much study.' And the travels of Polybius in all the countries of the Mediterranean, and in Gaul and the Atlantic, qualified him to write with full acquaintance of the local facts and of the knowledge which was preserved in the countries which he visited. Later writers may elaborate more fully one 
portion or another of this field of inquiry; but the principles and the spirit of history were perfectly apprehended, and Polybius must be said to have carried the system of written history to its complete development as a whole.

The next step came with the Renascence, when the older documents were sought out and history was compiled not only from what was personally known and in hand, but from early or even contemporary documents. These documents were in the first place copies of older historians. Then the contemporary evidences of inscriptions on stone and on coins were pressed into use. In the last generation or two, the older inscriptions of Egypt and Assyria have been brought into the scope of history, and we now read the contemporary accounts of the main events of from three to six thousand years ago. At this stage it might be supposed that our knowledge must come to an impassable barrier, and that beyond the reach of written record we cannot stretch our vision.

The new conception, which perhaps first came obviously forward in the discoveries of prehistoric man, is that of materialized history in place of written history. The permanence of the traces of man and of the results of his acts and works has never been grasped till the present generation. Even to this day the sites of ancient cities and palaces are raked to pieces and destroyed in the search for inscriptions, regardless of the great amount of history shown in the material remains, often much wider and fuller than any that is recovered from inscriptions.

The first use to which material history is applied is the confirmation and illustration of what is already recorded. To produce something which tallies with the statements of an author-an Assyrian cylinder s. L. 
naming Hezekiah, a Greek vase with a scene of the Odyssey, a coin with the Britannic triumph of Claudius -is felt to be so satisfactory to the reader who has trusted all his life to a printed page, that this use of material history has blocked the way to its real importance. These confirmations are the least important use of material.

The next use of material is to fill out and consolidate the fragmentary statements or bare outlines; such is the use of the Anglo-Saxon jewellery and weapons; the burnt ruins of the Roman towns in Britain, and the piteous cave-shelters in which their inhabitants took refuge from the heathen flood of barbarians; the primitive Roman inscriptions on the buried pillar of the Forum; the Greek settlements on the Euxine; and the filling of the outlines of Egyptian history with a living picture of the wealth, art, and civilization of the thousands of years that passed.

But the most valuable result from material history is the extension of it to ages before the written record of each country. So soon as man becomes a settler, and acquires anything beyond the skin and wood vessels of the nomad, he begins to lay by history; so soon as he disturbs the surface of the land by roads, entrenchments, or fields, he leaves the proof of his industry to the future; so soon as he even breaks a stone by skill and design he leaves an imperishable trace of his abilities. There is no land in which civilized man has lived, in which we cannot reconstruct his history entirely from his material remains.

At first sight any one accustomed solely to get ideas from words may be at a loss to see how much can be learned. The fact that certain towns, buildings, and roads existed is easily obvious. The mental capacities 
of a people are shown as clearly by their art as by their literature; the sense of beauty, the sense of accuracy, the power of expression, the amount of wealth and taste, are all shown by the decorations and the small objects. The foreign trade is shown by the importations from abroad. Even the details of organization leave their traces; in a group of 400 similar statuettes which I found, seventeen different styles were noticeable, but the styles vary greatly in number and are all irregular: hence even in a very large order such as this the work was done in a joint factory, and not let out to different men. The relative precision and truthfulness of work are continually shown in all constructions, and form a very exact test of one kind of civilization. We may even see-as in the pyramid of Cheops-at what point the master-mind of the chief architect passed away, and less precise work was done by his successor.

Not only can we thus gather a picture of the condition of a people at a given stage, but the actual historical changes can be traced. Every detailed map of England embodies the main changes of the history of the people. The growth of a town is always shown by the position of its streets and public buildings: the old lines of its walls are visible and the successive stages of its growth. Its relation to the roads show which roads preceded it, and which were made after the town. The relative ages of towns are thus shown by their relation to the roads which join them. The age of enclosure and cultivation of the country is shown by the relation of the field enclosures to the roads. And the old centres of life which have long since sunk into silence, before even the coming of the Romans, are still shown by the straight lines of field paths running across miles of country from one such centre 
to another. If we had nothing whatever but the map of England, on the scale of six inches to the mile, we might construct from it a more detailed history of the settlement and growth of the population, and the economic history of the country than has yet been written.

It is also possible to reconstruct long ages of the social history of a people without a single word of writing, entirely from the material history. Suppose, in some great country house, a room was closed, furnished, just as it stood, on the occasion of the death of each owner, there would be a series of roomfuls of furniture of each generation. No one could possibly suppose that the room of the age of William IV came between those of Anne and George I, or the room of Charles II between George III and IV. The changes of fashion would unquestionably link the rooms together in the right order when their contents were compared. Now this has been done with the groups of pottery found in graves. Some 900 graves of the prehistoric age in Egypt have been compared, and can be replaced in very nearly their right order. They form a chain of changes of fashion, and we can thus date the graves relatively in what we may call sequences or sequence dates, though not in absolute years. The next step is to date each kind of thing found in the graves by its first and last appearances, named in the sequence dates of the graves, then all other objects found with these also get their sequence dates. In this way wherever we have enough examples of groups of objects which are found together we can reconstruct their order, and so form a system of dating, in sequences though not in years.

The previous instances show how much is preserved of the history of one place or land as studied in itself. 
The other results of material history are not (I) local, but either (2) connected, as in the evidence of two different civilizations being contemporary, or (3) allusive, as in the artistic influence of one country upon another. The most complete instance of the connexion of one civilization with another is that of Egypt and Greece. The age of the Ptolemaic connexion has always been well known, and in the last twenty years there have been brought to light the early historic Greek settlements of Naukratis and Daphnae, the great so-called Mykenaean civilization of 1500 B.c. linked with Egypt, the earlier ' middle Minoan' civilization of Crete contemporary with the twelfth dynasty of Egypt about 2500 B.c., the 'early' Minoan' age with traces of the pyramid builders of Egypt 4000 B.C., and the neolithic periods of Crete connected with the first dynasty and prehistoric times of Egypt 4500-6000 B.c. ${ }^{1}$ Another instance of connected history is that of Gaul with Greece and Rome.

The allusive results are those which prove an influence of one country on another by the evidence of artistic taste and of actual patterns and motives of ornament. It is probable that the spiral ornament of the prehistoric age of Europe was imported into Egypt about 3000 B.C. The naturalistic style of Cretan art undoubtedly influenced Egypt largely about I 400 B.C. The Egyptian ideas and motives were reflected in the Mediterranean in the attitudes of Greek sculpture and the insular coinage (Malta, \&c.) at 600 B. C. The Assyrian style penetrated largely into the Euxine, and had a share later in the Greek style of that region. It was also carried by Phoenician trade strongly into Etruria and appears

1 The details of these connexions are given in Methods and Aims in Archaeology, pp. 141-68. 
commonly in the later prehistoric Italian work. The northern style seen in the silver work of Scandinavia is a main element in the Dacian work, which also partakes of Persian and Central Asian motives. A good instance of this is the celebrated 'Treasure of Petroassa'. The Greek style gave the great impetus to Indian work, and shows materially the Greek civilization of the Bactrian kingdom whose coins slowly filter down from pure Greek to pure Indian designs and inscriptions. And the Greek influences on Europe in the Middle Ages and down to our own times are ceaseless and all-pervading.

The history of artistic influence is an immense subject still awaiting study and classification; but it will be seen to form an important part of the material history of man. We may perhaps sum up by saying that material history is the only trace left of far the greater part of man's development and duration: it is quite on a par with written history in ages where both are preserved, so far as the whole of a people are studied as a community : and the only peculiar province of written history is in dealing with individual character and influence. In the socialist view of history the material history is far more important than the written record as a whole; in the individualist view the written record is unapproachable, as dealing with the influences of the exceptional minds which advance the frontier of ideas. Each has its fit place, and each is entirely powerless in the special region of the other means of research. The whole past of man during hundreds of thousands of years, down to the little clear fringe bordering on our own times, is entirely the province of material history; and even down to our own age it shares with written history that power of interpreting human action and change which is perhaps the most fascinating study that can engage our minds. 


\title{
IX
}

\section{SCIENTIFIC METHOD AS APPLIED TO HISTORY}

\author{
(The Very Rev. T. B. Strong)
}

THE previous Lectures in this course have dealt with the question of the application of scientific method in various branches of the investigation of the natural world. The majority of these separate sciences, as we call them, have for their object the establishment of laws of nature, or of general principles which will render possible the proper interpretation and the intelligent anticipation of the processes of nature. My present task is the very difficult one of inquiring how far the investigation of history may be pursued on scientific principles. This task appears to me difficult because, while much is said nowadays of the importance of a scientific conception of history, I do not think there is anything like the same agreement about the character of scientific history as exists in the case of most other sciences.

All science, we may say, consists in strengthening, solidifying, and rendering conscious and coherent the ordinary processes of knowledge. The scientific man makes no claim to an absolutely separate method of acquiring information, as if he had some exceptional gift of inspiration. He claims to clear away fallacies, to bring into clear light the real principles by which all men's knowledge is acquired, and to use these in 
a highly complicated way for the extension and co-ordination of knowledge. When we ask, therefore, what is the nature of scientific method as applied to history, we are at once thrown back upon two preliminary questions: What are the facts which history includes? and What are the ordinary processes of the mind by which we attain the knowledge of them? I shall consider these two questions first, and then proceed to inquire in what way the ordinary methods of knowledge may be so strengthened and cleared up as to give us what may be called scientific history.

I. In one sense, of course, any event occurring in the course of the world's movement may be a fact for history. The mole that threw up the mole-hill over which William III met his death is, as we say, historic: he appears in history as 'the little gentleman in black velvet,' whose health was enthusiastically drunk by Jacobites. But, ordinarily speaking, arts such as that of the mole's, and merely physical events, do not come within the purview of history. History concerns itself primarily with human acts, and with physical facts as they affect and are woven into the fabric of human life.

Now it is obvious that in dealing with historic facts in this sense it is easy to classify them-to trace similarities of various kinds in them, to represent them as illustrations of general tendencies. The murder of Hipparchus by Harmodius and Aristogeiton, the murder of Julius Caesar, the murder of the Czar Alexander II in 188I may all be described as cases of tyrannicide. We may, if we choose, draw comparisons between the political circumstances which lead to such acts, and trace parallels and contrasts between the various cases. So far as history aims at setting out the general laws which govern human life, such processes must be applied to 
the historic data. From this point of view there is a somewhat close analogy between history and other forms of scientific investigation. Given the facts, the scientific historian endeavours to trace the operation of principles in them. But this reflection only leads on to the statement of a point in which historic data differ very widely. from the data of other sciences, especially the physical sciences. For the purposes of history every fact is unique, i.e. is fettered in place and time: for the purposes of physical science every fact is potentially independent of particular place and time, and every explanation of a fact is potentially of universal application. Thus if I want to investigate the nature, say, of the sunrise, I start perhaps from some instance which I may have observed: but the explanation, if I find it, is valid of every single instance since the creation of the world, and for all future instances so long as the solar system continues to exist. And any one of these instances would have done as well as any other to serve as the starting-point of my investigations. This is not the case with the other events just mentioned. I cannot infer from the rule that certain political conditions tend to produce tyrannicide (supposing, for a moment, I am justified in assuming this rule to be true), even that Hipparchus or Julius Caesar or Alexander II was killed in point of fact: still less that the event occurred on any particular day or in any particular conditions: whereas, if my explanation of to-day's sunrise is sufficient, I can infer that the sun must have risen at a calculable time on any day in the past history of the world. Such laws as we reach by the investigation of history do not enable us to infer the historic occurrence of any given fact or the conditions of it. They are generalizations rather than laws. Thus facts 
for history are past, and unique: and the object of historical evidence will be to make clear to our minds that some particular event has actually taken place. The form of an historical statement is as follows: at such and such a place and time such and such an event occurred, or so and so performed such and such an act. And the question scientific history has to answer is this: Under what conditions am I justified in believing these historical statements?

At this point I would refer for a moment to the interesting and brilliant lecture given by Mr. Flinders Petrie in this room yesterday. He used words to this effect: if all the written history of London were swept away, it would be possible to reconstruct the history by means of the material recovered by investigation. His concluding words partially restored my confidence in my own lecture: for they showed that there is a wide distinction between what he called social and individual history: the former deals with the sequence of stages through which a people has passed, and may be written in general terms without individual names, dates, or events. Individual history deals with the particular details of name, date, and event through which the social changes expressed themselves. I am concerned to-day with these only. I want to deal with the facts of ordinary experience as they occur, and to inquire into the mental condition of persons who believe facts which they did not themselves experience; and the question is mainly a logical one.

Before we pass on to the question of the mental attitude towards historical affirmations, I wish to mention a point which is of some importance, owing to a somewhat persistent habit of common speech. It is very commonly assumed that there is a close parallel between 
legal and historical evidence, so that a person who is practised in the Courts will probably be a good judge of history, and what could not be proved in the law-courts is probably unsound historically. The only point, so far as I can see, at which there can be any possibility of contact is in the investigation of facts as they come before a jury. The judge explains the law: if A. B. did such and such acts then he comes under the law of murder or libel or whatever else it may be: it is for the jury to find whether he did them or not. But it seems to me that, as compared with the questions raised in history, legal questions are of extraordinary simplicity. In a legal case, what has happened is usually admitted. A murder has been done : an article has been published. There is no doubt about the facts, and no doubt about the person accused of being responsible for them. In some cases the problem is to connect the party accused with the facts by links of the particular cogency which the law requires. At other times the agency of the accused is admitted, and the sole question is to estimate the degree of his responsibility or the existence in his mind of malice. In other words, the points which the law starts with have to be proved and established by the historian : and the latter will be at different times more and less exacting than the lawyer. The law would run the risk of letting off a guilty man rather than of condemning an innocent one: history cares only to establish the truth by any means in its power; in this sense it is relentless. The one deals with isolated facts and definite persons: the other may range indefinitely over a field in which acts and agents are all alike uncertain.

II. I have endeavoured to indicate as clearly as I can the form of the historical statement and the historical 
question: I now wish to consider shortly our mental attitudes towards historical statements. Let us consider the question first in connexion with some very obvious and ordinary conditions. As we pass through the London streets we see placards with notices of the following sort: Great Colliery Disaster : Return of the King to London : Triumphant Government Majority: or in papers of a slightly different complexion-Latest Tory Shuffle. Being accustomed to these placards we are often contented with the headlines, and do not buy the papers: if, however, our interest is strongly excited, we wish to know more fully about the events; we pay our money, and get the paper. Then we find that these headlines are the titles of a number of short historical works, which we can read at length in the paper. We find that the two last describe the same division in the House of Commons : under the head of the king's return to London we shall probably read of red cloth and General Managers, kindly and pleasant words from His Majesty, perhaps an escort of soldiers, a rapid drive to Buckingham Palace. The account of the colliery disaster will tell of sorrow and heroism, and public sympathy, in the order which we all know so well. Now I have not been present at any one of the events described; in their relation to my mind they are as truly fragments of history as the Battle of Marathon. I read the accounts, however, in the papers, and I have no hesitation in believing them immediately: I assume that they have been truly reported: I talk about them, if so moved, to others, assuming that they have read and believed them like myself. I am haunted by no qualms of doubt: I do not attempt to verify the statements: I simply take them as they are. Supposing, however, I find all these statements, or some of them, contradicted in the next morning's issue, I retain 
my mental equilibrium. I am very glad there was no colliery disaster : the report of it must have been like the report of the massacre at Pekin some years ago, which turned out false. I reconcile myself to the idea of the king being still out of town: to a different view of the division in the House of Commons. And this also occurs without much searching of heart, and without any attempts at verification.

Why is this? It seems to me to depend mainly on the fact that all these events belong, so to say, to the natural context of my experience. All lie outside the limits of any direct observation on my part, but they are all things which I am aware may happen : the possibility of them is, so to speak, a suppressed or unconscious presumption in my mind: it requires little more than an assertion, in one of the regular channels of current history, for me to accept the statement: and the denial is scarcely more difficult. Though these things are easy to accept, there is no reason why they should have happened, and rumours are not invariably true. They do not exactly require verification or elaborate criticism, because the experience to which they belong, in its wider aspect, is a perpetual process of criticism and verification in itself. In short, statement and denial are equally easy to accept, because either will fall readily into the context of my ordinary experience. In one sense, I can never prove any of the statements : that is, I can never avoid the fact that I depend for my knowledge of them upon the knowledge and veracity of others. But, knowing the way things mostly go in England, I know that I can fairly trust the news in the daily papers.

This, however, is not quite all that should be said. To resign with so little compunction a statement accepted without any criticism implies a somewhat low form of 
belief. On the whole, I may attain a fairly true picture of contemporary life, but it is not scientifically based. In every other relation a thing which we seriously believe requires serious efforts to dislodge. If we have accepted a statement on the basis of evidence carefully examined and tested, it will take at least as much care to allow us to arrive at an opposite conclusion. I should, therefore, venture to make a distinction between the mental attitude in which we can be really said to believe an historical statement, and that (by far the commonest) in which we acquiesce in a particular statement so long as it remains uncontradicted. Such acquiescence may be due to various causes. It may be due, for instance, to indifference, or to the sense of the difficulty of examining and verifying all statements with which we are confronted, or to the conviction that experience will gradually organize itself: but whatever its cause, it is a very common state of mind: it may pass into conviction if the circumstances permit, but it falls short in itself of definite historical belief. It is, I think, of importance not to confuse these two states of mind. As an early stage of scientific historical certainty it is well described by Browning :-

\section{Call belief}

Belief indeed, nor grace with such a name The easy acquiescence of mankind

In matters nowise worth dispute, since life Lasts merely the allotted moment ${ }^{1}$.

We must now come somewhat more closely into connexion with our subject. We have learnt what is the usual form of historical statements, and distinguished two

${ }^{1}$ Ferishtah's Fancies, 'Shah Abbas' (Poetical Works, ii. 659). 
mental attitudes towards them, that of easy acquiescence, and that of serious historical belief, and we must now ask by what methods and arguments can we establish the condition of historical belief, in cases where there is reason for doubt or hesitation.

In order to answer this question, let us turn back to the instances of historic statement which we have just considered-and let me point out that the mere fact that they are modern and ordinary is not sufficient to distinguish them from other facts in history. They occur outside our experience, and we have to depend upon human testimony in order to get at them. In the present day we have special arrangements for the quick spread of intelligence : a battle in Manchuria is reported in London almost as soon as it is over, but less than a hundred years ago it was some days before the Battle of Waterloo could be known. The rapidity with which we now gain information in some measure conceals from us its character: it is historical, however, and our attitude towards it is by no means unimportant for our purpose. There is, nevertheless, a real difference between these items of daily intelligence and the facts of history for which we depend on documents, and it lies in a point to which I have already called attention. In the modern cases we are careless and indifferent in verification; we are ready to accept the testimony to hand because the whole series of facts alleged to have occurred belongs to a context which we, in large measure, understand. Though the facts were outside our direct observation, yet we live in the context in which they occurred. We know the various elements in it: the scenery and furniture are familiar: we know the probabilities: we have a general view of what is likely and what is not. And nothing marks more strikingly the effect of modern 
scientific appliances than this, that the whole world is now in our context.

If in any period of more distant history we had similar knowledge of the environment, we could deal no less easily with the historical statements which come down to us out of the past. But this is just what we never have. Events occur, and the people who are contemporary with them either have, or can easily acquire, the links which connect them with the rest of the world of the day. But time as it goes on drops endless numbers of these links, and after a certain number of years certain events seem to stand out, isolated and remote, as rocks stand out of the monotonous surface of the sea. It is the task of the historian-especially the scientific historian-to recreate, as far as he can, the lost context of these remote facts, to find their necessity a posteriori, to show how inevitably they are involved in the present-no a priori necessity, as we have seen, can be shown in history - to make the ancient period alive and coherent. No one can do this with absolute success, though here I should like to remind you of the enormous debt which history owes to the archaeologists. It is almost certain that there will remain always some details which seem disconnected and irrational; but the man who has really mastered and steeped his mind in the history of some one period will have some of the skill of a contemporary: he will be less likely to be deceived by false lights, and he will have intuitions as to what probably did occur, which are in large measure worthy of trust. No man can value adequately, or even grasp enough to believe in, what he does not understand: the historian's purpose is to make the past credible by making it intelligible: to show its effects in the present which we know, if that is possible: 
to eliminate the false ideas which lack of intelligence and feeble imagination have developed around it.

The main functions of the historian may, I think, be inferred from the general outline just given. The historian, in his examination of the past, like the reader of the newspaper in the present, has to deal with witnesses; but his witnesses are not persons whose general character is easy to estimate, like the newspaper reader's, but people, often long since dead, often anonymous, whose character and aims have to be painfully 'restored' out of a number of fragmentary remains. That is, he has to criticize his authorities. And, again, he has to form a picture of the whole historical environment in which his authorities lived and thought, else he is continually liable, in dealing with them, to impose tests which are anachronistic: and this means that he must criticize very carefully all the documents which can be said to bear upon the period. We are to-day trying to think of history in abstracto as a scientific process, a means of attaining truth. This is peculiarly difficult in a matter in which details and particulars form so large a part of the subject; and I am aware that any treatment of the question must leave much to' be desired. It will be impossible in the time at my disposal to go into all the various elements of discussion which belong to a complete treatment of historical science. But in this connexion I would commend to your notice a work by two French Professors at the Sorbonne, MM. Langlois and Seignebos, An Introduction to the Study of History. The work is translated into English, with a Preface by our late Professor York Powell. I will content myself with references to a few only of the points involved.

Every form of scientific investigation must begin with some facts or beliefs, assumed or proved to be certain. 
That is, we cannot for the purposes of scientific reasoning begin from a universal doubt. But this does not necessarily imply that no investigation has gone to the formation of the premisses whatever they are. One science will, frequently, take from another the premisses with which it proposes to begin. So, though the interlocking of the various interests is, in the present case, more intricate than usual, we may perhaps assume that historical science will take over from archaeology or from palaeography some fixed starting-points in the way of dated documents. The historian will collect and examine (as Langlois and Seignebos have pointed out) the whole mass of documents concerned with his period: he will find out exactly what they say: he will reject manifest contradictions and anachronisms. Incidentally, by this process a provisional picture will grow in his mind of the history of the period: he will begin to understand the coherence and the drift of it: he will be getting into the frame of mind in which he can bring his conception of the period to bear upon particular statements of individual authorities. All this process is described in considerable detail by MM. Langlois and Seignebos. But I venture to think that this is, logically speaking, prior to the asking of the real historical question: Can I believe this historical statement, and why? What really has to be settled when this question is raised at last is the value of the authority, and this is a particularly difficult question to answer. All we can ever do in regard to it is to reach the result: "The statement or statements of such and such an authority may be trusted.' In regard to this point, I venture to differ in some degree from the two authors to whom I have referred: I should attach more value than they seem to do to the character of the authority. The great source of the difficulty of history altogether is the presence of 
the human element. This is one main reason why there is so little room for prediction in dealing with it: we cannot tell what men must have done, we can only learn indirectly what they have done. While we deal with documents in ways hinted at above we use methods which follow almost mechanical rules. We analyse, compare, and classify their statements : we fix their dates by various processes of more or less certain validity: and we obtain, as a result, a sort of provisional view of the state of things in the period. Many of the documents before us will probably be formal state-papers, records made in the way of business, and so on. If we are persuaded that these genuinely belong to the period they claim they have a high degree of authority. But a large portion of the documents under discussion will be due to the voluntary enterprise of individuals, and it is in these that we shall find the most intimate and detailed account of things. It seems to me that, whereas it is laborious but comparatively easy to estimate formal documents, it is difficult to estimate the value of independent voluntary records, because much will depend on the character, opportunities, and capacities of the writer. We cannot do without chroniclers and historians if we are to have anything like a full account of the past, but it is of vital importance to us to know whether they are trustworthy witnesses or not.

This judgement of our authorities must be of gradual growth, and is made up of many considerations. We must, for instance, note the conditions, so far as they are revealed, in which our author claims to write, and, even more important than that, the indications which he supplies (unconsciously) of his way of working and his conception of a fact. So far as it is possible, we must endeavour to distinguish between the real record of 
actual experience and the dramatic adoption of the tone of an eye-witness. And we shall test our authors as far as may be in connexion with statements of fact of which there is the fullest independent evidence. For this will enable us most clearly to distinguish the psychological characteristics of a given author, and attain some notion of his general credibility. If all goes well, we may arrive at last at a picture of the events concerned in which the various strains of evidence fit in together, or at least define themselves with sufficient clearness to be compared and weighed against one another; and in which the contents of their evidence form something like a rational and coherent whole. If to this can be added the fact that further investigation down the line of history more and more obviously runs back upon a view of the world such as we have derived from our estimate of the evidence, we shall have the best reason that can be given us for accepting the statements of our authorities as true, or for trusting those whose statements build up the coherent picture, and distrusting, on the whole, those which are eccentric, or which have already been discredited under examination.

I have struggled vigorously to set out my views of these processes in abstract form, avoiding, as far as possible, any side-glances at particular instances, because it appears to me that in this way the process of reasoning becomes clearest. If I have been right in my exposition, it follows that there are, as it were, two elements in historical belief : the acceptance of statements on the authority of witnesses, in whom we have confidence, and the partial verification of these statements by comparison with others, on the assumption that in ordinary cases the chances of misplacement of confidence will gradually be eliminated if a sufficient variety of testimony 
can be got. This result, perhaps, seems painfully obvious, but I would urge that it is also rather startling. For it means that when we really believe a historical statement we yield credence to the character of some man whom we have never personally known; and that things being as they are, no historical statement can be so completely proved beyond all possibility of cavil as to dispense us from the necessity of this act of confidence in human testimony. But I should go further than this. When we come to think of it, it is the few rather than the many among historical statements which permit of verification. Some have entered so largely into the fabric of history that the chances of their being false are comparatively slight. For instance, the fact of the murder of Julius Caesar is as nearly indisputable as a fact can be, because of the variety of ways in which it is involved in history, and its connexion with the whole plan of things as we understand it. But the statement that he died on the Ides of March, or by so many wounds, must be accepted, if at all, on the strength of the main fact and the generally excellent character of our authorities on the subject. No doubt all these details have their link with the main fact, and, if we knew all, might be seen to have been inevitable. But all their links with the general order of things are gone. We can never be said to have proof of them. To us they do not matter. We may believe them on the strength of the character of those who report them, or we may adopt towards them the attitude of easy acquiescence which we adopt towards so much of contemporary history.

I have laid emphasis upon the element of acceptance of human testimony in my exposition, because this seems to me the distinctive mark of our relation to historical as opposed to other evidence. And I would call attention 
to the fact that this is precisely suited to the treatment of a body of facts, all of which are unique in space and time. These facts, as we have seen, are outside our own experience: they cannot be presented as the result of a deductive process : and the only way in which we can assure ourselves that such and such an event occurred at a certain place and time is by accepting the testimony of some one who knows. Human testimony is uncertain : in recent years we have had a controversy as to the exact mode in which Charles I was beheaded; this year there is an animated discussion as to the details of Nelson's tactics at Trafalgar. We can never be absolutely sure of our testimony, but it is the best thing we have.

If it be contended that this is a sceptical view of history, calculated to destroy much of our confidence in it, I would venture to urge that this contention arises in consequence of the prevalence of a false ideal of proof in history. In the mathematical and physical sciences proof is complete and final ; the conclusions are free from ambiguity or from doubt. But the possibility of such proof depends absolutely on the conditions in which these sciences work: their ideal belongs to them in virtue of the character of the facts they investigate and the abstract method pursued: it cannot be transferred to any other situation. The physical sciences deal with aspects of physical events which are continually increasing in the degree of their abstractness: the confused and detailed fact of daily experience is further and further from the purview of physical science; but history, as we have seen, has to do with the ordinary fact of daily experience, and cannot even abstract it from the relations of time and space. Historical facts are susceptible of a proof that is suited to them, and no more; and the proof will always look painfully precarious and inadequate to one whose expect- 
ations of what proof should be are based upon the experience of physical science only.

This is one point in which physical science seems to me to supply the historian with a false ideal: I venture to think there is another. It is arguable, and there are many who maintain the position, that the formulae which make possible the most comprehensive generalizations are formulae only-theories which colligate the phenomena, but which do not profess to be-to use ordinary phrases -true of the outer world. Professor James Ward, for instance, maintains this view about the fundamental conceptions of physics. He maintains, i. e., that the atoms and the molecules are not things like tables and chairs only smaller-not objects of a possible experience-but formulae of a highly abstract kind. There is no harm in this, if it be true: it does not make physics any less valuable as a science, it precludes only statements and inferences which assume the external reality of these ideas. If another series of formulae could be devised which was equally useful for co-ordinating experience, we could drop the old and embrace the new without any searchings of heart. It appears to me that historical critics are apt to suggest theoretical explanations in the region of history in somewhat the same spirit, and to show by so doing that they have forgotten the uniqueness in space and time of historical facts. Hypothetical authors are freely proposed for parts of books of which the whole drift may be hard to grasp ; and it is tacitly assumed that in historical investigation plausibility and convenience have the same weight as in physics. This also appears to me to be an unfortunate imitation of physical science. The historian's work is only begun when he is in face of a variety of plausible theories: he has to show which of them is true and why. And the presumption is always in 
favour of the man who requires no conjectural authors or other hypothetical entities.

I have suffered in this lecture from a limitation which has affected all who have taken part in this course-the necessity of forcing into a limited space matter that should occupy a wide series of lectures. And in one respect it seems to me that the discussion of historical evidence must necessarily be somewhat disappointing as compared with the other forms of scientific knowledge. The other lecturers have shown how successfully their science has proved something: I have been bound to dwell on the extreme precariousness and limited range of certainty belonging to history. The logical type of a proved historical fact is not a clear-cut argument moving to an inexorable conclusion, but a complicated exposition of a great variety of inconclusive considerations, out of which certainty emerges gradually by a process of accumulation. For many facts recorded no unassailable proof is possible at all; but those facts which we may regard as proved, from the variety of their evidence and the wide area of their effect, do extend a kind of indirect certainty over others, unprovable otherwise, which are linked with them and depend upon them. And behind all this lies the question of the value of human testimony. We know it to be uncertain : any of our witnesses may be untruthful or himself deceived; and it is difficult to determine the truth about them. No laws of probability will protect us against involuntary error: we can only correct that by some a posteriori critical process. But on the whole it is probably true that men do not, except for motives which are more or less calculable and transparent, aim at deceiving their fellows. And if you ask why we should believe any statement which we cannot verify, it appears to me that you must fall back on this fact-which is in truth only 
another form of the solidarity of mankind. St. Paul puts what seems to me the principle succinctly, when he says, 'Putting away lying, speak every man truth with his neighbour, for we are members one of another.' On the whole the race has an instinct for, as well as an interest in, truth which we can trust-not credulously or irrationally, but reasonably and carefully. We shall be deceived from time to time, and make mistakes ; but we must make our venture, for without it we must be content with the very limited knowledge of the world that directly reaches our own eyes and ears. 
OXFORD

PRINTED AT THE CLARENDON PRESS BY HORACE HART, M.A. PRINTER TO THE UNIVERSITY 



\section{RETURNTO: ANTHROPOLOGY LIBRARY 230 Kroeber Hall $642-2400$}

\begin{tabular}{|c|c|c|}
\hline LOAN PERIOD 1 & 2 & 3 \\
\hline 4 & 5 & 6 \\
\hline
\end{tabular}

All books may be recalled. Return to desk from which borrowed. To renew online, type "inv" and patron ID on any GLADIS screen.

\section{DUE AS STAMPED BELOW.}

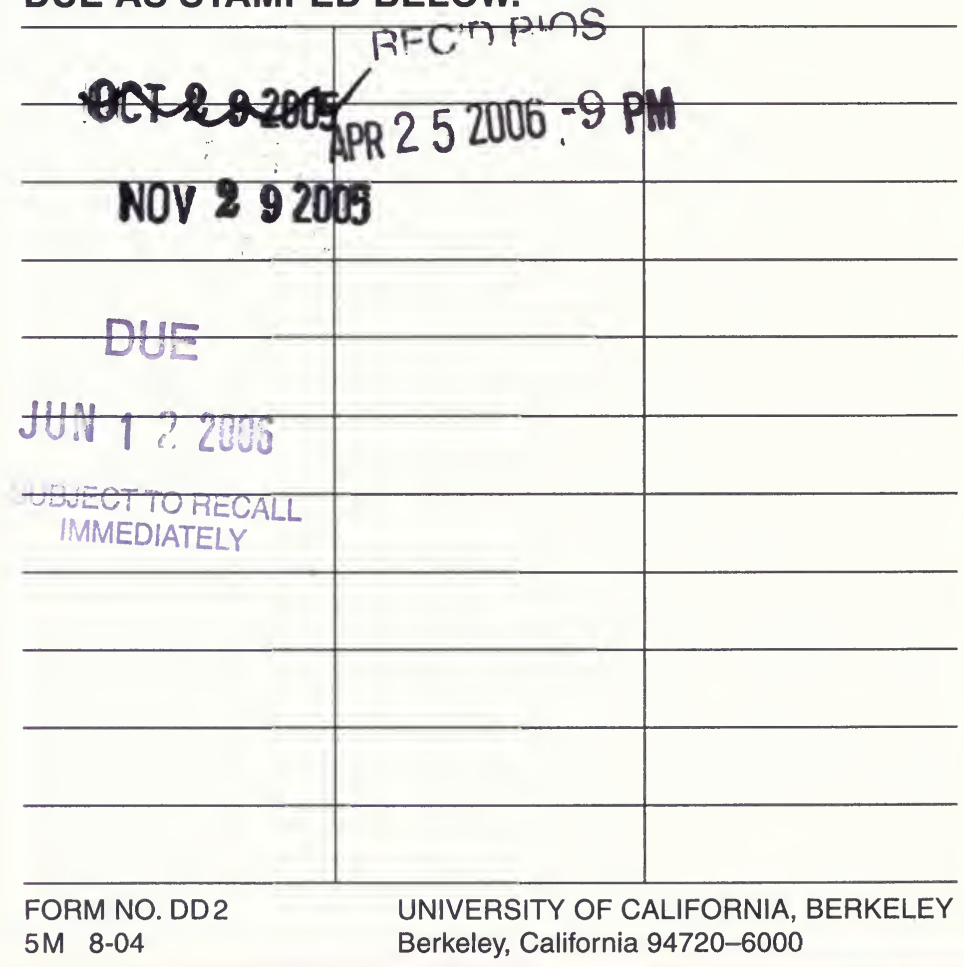




$$
\text { YC } 22522
$$

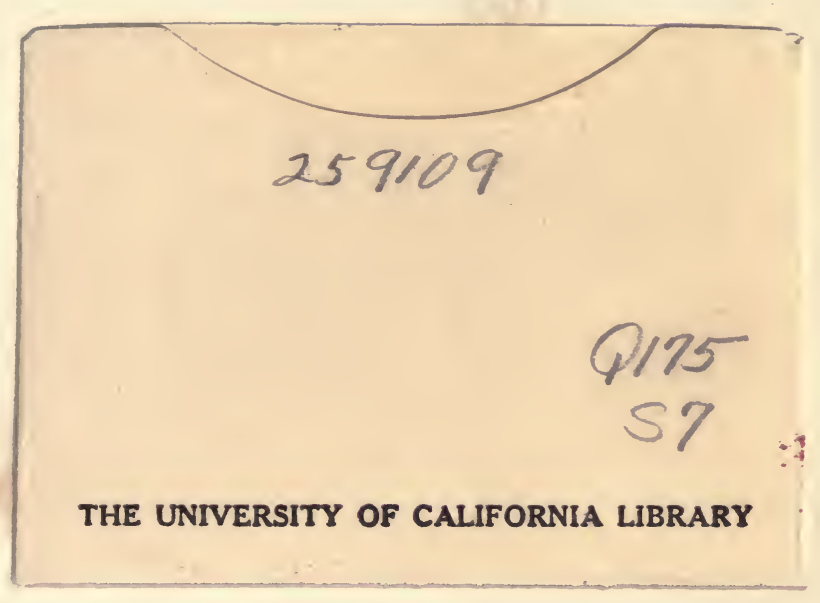


$\sin 20$

8

nogse

(3)

15.20

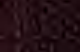

Sops

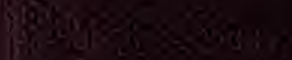

